\title{
Chiral boron enolate aldol additions to chiral aldehydes
}

Luiz C. Dias and Andrea M. Aguilar

${ }^{a}$ Instituto de Química, Universidade Estadual de Campinas, UNICAMP, C. P. 6154, CEP 13084-971, Campinas, SP, Brazil

ldias@iqm.unicamp.br

\section{Supporting Information}

\begin{tabular}{|c|c|}
\hline CONTENTS & PAGE \\
\hline${ }^{1} \mathrm{H}$ NMR spectrum (300 MHz, $\mathrm{CDCl}_{3}$ ) of aldols (16) and (17) & SI-4 \\
\hline${ }^{13} \mathrm{C}$ NMR spectrum (75 $\mathrm{MHz}, \mathrm{C}_{6} \mathrm{D}_{6}$ ) of aldols (16) and (17) & SI-4 \\
\hline $\begin{array}{l}\left.{ }^{1} \mathrm{H} \text { NMR spectrum ( } 300 \mathrm{MHz}, \mathrm{C}_{6} \mathrm{D}_{6}\right) \text { of benzylidene acetal derived } \\
\text { from aldol (16) }\end{array}$ & SI-5 \\
\hline $\begin{array}{l}{ }^{13} \mathrm{C} \text { NMR spectrum (75 } \mathrm{MHz}, \mathrm{C}_{6} \mathrm{D}_{6} \text { ) of benzylidene acetal derived } \\
\text { from aldol (16) }\end{array}$ & SI-5 \\
\hline IR spectrum (film) of benzylidene acetal derived from aldol (16) & SI-6 \\
\hline HRMS of benzylidene acetal derived from aldol (16) & SI-6 \\
\hline${ }^{1} \mathrm{H}$ NMR spectrum (300 MHz, $\mathrm{C}_{6} \mathrm{D}_{6}$ ) of a mixture aldol 18 and 19 & SI-7 \\
\hline${ }^{13} \mathrm{C}$ NMR spectrum (75 $\mathrm{MHz}, \mathrm{C}_{6} \mathrm{D}_{6}$ ) of a mixture of aldols 18 and 19 & SI-7 \\
\hline${ }^{1} \mathrm{H}$ NMR spectrum (300 $\mathrm{MHz}, \mathrm{C}_{6} \mathrm{D}_{6}$ ) of aldol (23) & SI-8 \\
\hline${ }^{13} \mathrm{C}$ NMR spectrum (75 $\mathrm{MHz}, \mathrm{C}_{6} \mathrm{D}_{6}$ ) of aldol (23) & SI-8 \\
\hline DEPT 90/135 (75 $\left.\mathrm{MHz} \mathrm{CDCl}_{3}\right)$ of aldol (23) & SI-9 \\
\hline IR spectrum (film) of aldol (23) & SI-9 \\
\hline HRMS of aldol (23) & SI-10 \\
\hline
\end{tabular}




\begin{tabular}{|c|c|}
\hline${ }^{1} \mathrm{H}$ NMR spectrum (300 MHz, $\mathrm{C}_{6} \mathrm{D}_{6}$ ) of aldol (24) & SI-10 \\
\hline${ }^{13} \mathrm{C}$ NMR spectrum (75 $\mathrm{MHz}, \mathrm{CDCl}_{3}$ ) of aldol (24) & SI-11 \\
\hline DEPT 90/135 (75 $\left.\mathrm{MHz} \mathrm{CDCl}_{3}\right)$ of aldol (24) & SI-11 \\
\hline IR spectrum (film) of aldol (24) & SI-12 \\
\hline HRMS of aldol (24) & SI-12 \\
\hline${ }^{1} \mathrm{H}$ NMR spectrum (300 MHz, $\mathrm{C}_{6} \mathrm{D}_{6}$ ) of acetal (25) & SI-13 \\
\hline${ }^{13} \mathrm{C}$ NMR spectrum (125 $\left.\mathrm{MHz}, \mathrm{C}_{6} \mathrm{D}_{6}\right)$ of acetal (25) & SI-13 \\
\hline DEPT 90/135 (125 MHz, $\left.\mathrm{C}_{6} \mathrm{D}_{6}\right)$ of acetal (25) & SI-14 \\
\hline IR spectrum (film) of acetal (25) & SI-14 \\
\hline HRMS of acetal (25) & SI-15 \\
\hline${ }^{1} \mathrm{H}$ NMR spectrum (300 MHz, $\mathrm{C}_{6} \mathrm{D}_{6}$ ) of aldol (26) & SI-16 \\
\hline${ }^{13} \mathrm{C}$ NMR spectrum (75 MHz, $\mathrm{C}_{6} \mathrm{D}_{6}$ ) of aldol (26) & SI-16 \\
\hline DEPT 90/135 (75 MHz, $\mathrm{C}_{6} \mathrm{D}_{6}$ ) of aldol (26) & SI-17 \\
\hline IR spectrum of aldol (26) & SI-17 \\
\hline HRMS of aldol (26) & SI-18 \\
\hline${ }^{1} \mathrm{H}$ NMR spectrum (300 MHz, $\mathrm{C}_{6} \mathrm{D}_{6}$ ) of acetal (27) & SI-18 \\
\hline${ }^{13} \mathrm{C}$ NMR spectrum (125 MHz, $\left.\mathrm{C}_{6} \mathrm{D}_{6}\right)$ of acetal (27) & SI-19 \\
\hline DEPT 90/135 (125 MHz, $\left.\mathrm{C}_{6} \mathrm{D}_{6}\right)$ of acetal (27) & SI-19 \\
\hline${ }^{1} \mathrm{H}$ NMR spectrum (300 $\mathrm{MHz}, \mathrm{C}_{6} \mathrm{D}_{6}$ ) of aldol (28) & SI-20 \\
\hline${ }^{13} \mathrm{C}$ NMR spectrum (75 MHz, $\mathrm{C}_{6} \mathrm{D}_{6}$ ) of aldol (28) & SI-20 \\
\hline IR spectrum of aldol (28) & SI-21 \\
\hline HRMS of aldol (28) & SI-21 \\
\hline${ }^{1} \mathrm{H}$ NMR spectrum (300 MHz, $\mathrm{C}_{6} \mathrm{D}_{6}$ ) of compound (29) & SI-22 \\
\hline${ }^{13} \mathrm{C}$ NMR spectrum (75 MHz, $\mathrm{C}_{6} \mathrm{D}_{6}$ ) of compound (29) & SI-22 \\
\hline IR spectrum (film) of compound (29) & SI-23 \\
\hline HRMS of compound (29) & SI-23 \\
\hline${ }^{1} \mathrm{H}$ NMR spectrum (300 MHz, $\mathrm{C}_{6} \mathrm{D}_{6}$ ) of aldol (30) & SI-24 \\
\hline${ }^{13} \mathrm{C}$ NMR spectrum (75 MHz, $\mathrm{C}_{6} \mathrm{D}_{6}$ ) of aldol (30) & SI-24 \\
\hline DEPT 90/135 (75 MHz, $\mathrm{C}_{6} \mathrm{D}_{6}$ ) of aldol (30) & SI-25 \\
\hline IR spectrum of aldol (30) & SI-25 \\
\hline
\end{tabular}




\begin{tabular}{|c|c|}
\hline HRMS of aldol (30) & SI-26 \\
\hline 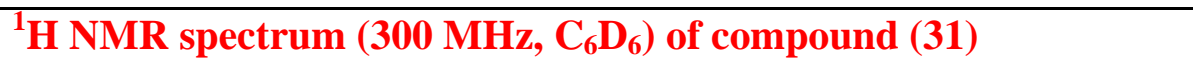 & SI-26 \\
\hline${ }^{13} \mathrm{C}$ NMR spectrum (75 MHz, $\mathrm{C}_{6} \mathrm{D}_{6}$ ) of compound (31) & SI-27 \\
\hline IR spectrum of compound (31) & SI-27 \\
\hline HRMS of compound (31) & SI-28 \\
\hline${ }^{1} \mathrm{H}$ NMR spectrum (300 MHz, $\mathrm{C}_{6} \mathrm{D}_{6}$ ) of aldol (32, major isomer) & SI-29 \\
\hline${ }^{13} \mathrm{C}$ NMR spectrum (75 MHz, $\mathrm{C}_{6} \mathrm{D}_{6}$ ) of aldol (32, major isomer) & SI-29 \\
\hline IR spectrum (film) of aldol (32, major isomer) & SI-30 \\
\hline${ }^{1} \mathrm{H}$ NMR spectrum (300 MHz, $\mathrm{C}_{6} \mathrm{D}_{6}$ ) of aldol (32b, minor isomer) & SI-30 \\
\hline${ }^{13} \mathrm{C}$ NMR spectrum (125 MHz, $\mathrm{C}_{6} \mathrm{D}_{6}$ ) of aldol (32b, minor isomer) & SI-31 \\
\hline IR spectrum (film) of aldol (32b, minor isomer) & SI-31 \\
\hline${ }^{1} \mathrm{H}$ NMR spectrum (300 MHz, $\mathrm{C}_{6} \mathrm{D}_{6}$ ) of aldol (33) & SI-32 \\
\hline IR spectrum of aldol (33) & SI-32 \\
\hline${ }^{13} \mathrm{C}$ NMR spectrum (75 MHz, $\mathrm{C}_{6} \mathrm{D}_{6}$ ) of aldol (33) & SI-33 \\
\hline${ }^{1} \mathrm{H}$ NMR spectrum (300 MHz, $\mathrm{C}_{6} \mathrm{D}_{6}$ ) of aldol (35) & SI-33 \\
\hline${ }^{13} \mathrm{C}$ NMR spectrum (125 MHz, $\left.\mathrm{C}_{6} \mathrm{D}_{6}\right)$ of aldol (35) & SI-34 \\
\hline IR spectrum (film) of aldol (35) & SI-34 \\
\hline HRMS of aldol (35) & SI-35 \\
\hline${ }^{1} \mathrm{H}$ NMR spectrum (300 MHz, $\mathrm{C}_{6} \mathbf{D}_{6}$ ) of aldol (36) & SI-35 \\
\hline${ }^{13} \mathrm{C}$ NMR spectrum (75 $\mathrm{MHz}, \mathrm{C}_{6} \mathrm{D}_{6}$ ) of aldol (36) & SI-36 \\
\hline IR spectrum (film) of aldol (36) & SI-36 \\
\hline HRMS of aldol (36) & SI-37 \\
\hline${ }^{1} \mathrm{H}$ NMR spectrum (300 MHz, $\mathrm{C}_{6} \mathrm{D}_{6}$ ) of aldol (37) & SI-37 \\
\hline${ }^{13} \mathrm{C}$ NMR spectrum $\left(75 \mathrm{MHz}, \mathrm{C}_{6} \mathrm{D}_{6}\right.$ ) of aldol (37) & SI-38 \\
\hline IR spectrum (film) of aldol (37) & SI-38 \\
\hline HRMS of aldol (37) & SI-39 \\
\hline
\end{tabular}




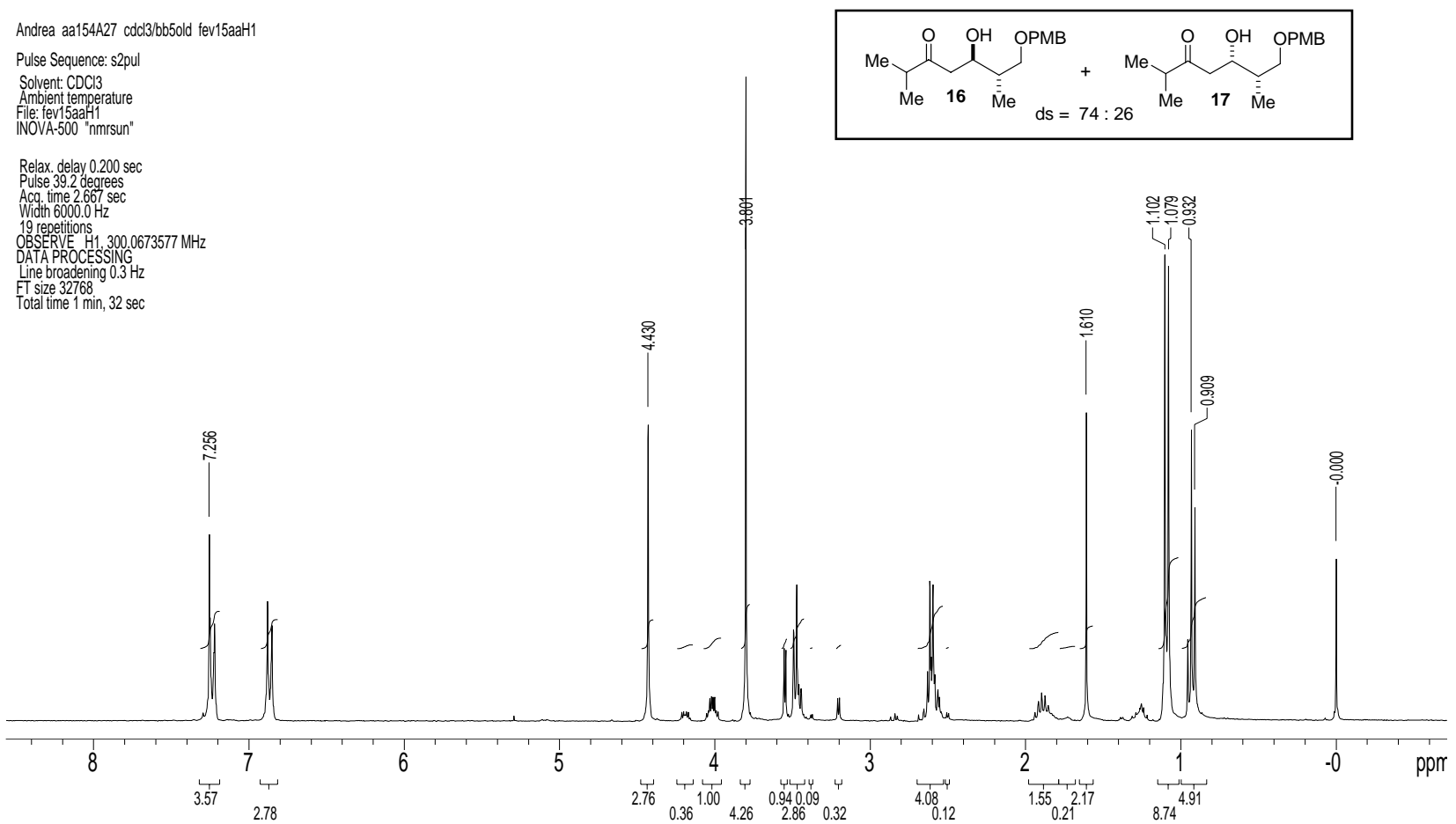

${ }^{1} \mathrm{H}$ NMR spectrum (300 MHz, $\mathrm{CDCl}_{3}$ ) of aldols (16) and (17)

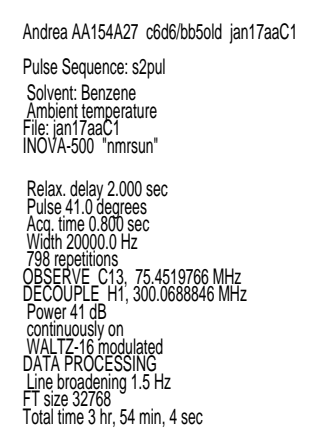

Andrea AA154A27 c6d6/bb5old jan17aaC1

Pulse Sequence: s2pul

Solvent: Benzene

File: 2 an17aaC1
INOVA-500 "nmrsun"

Relax. delay $2.000 \mathrm{sec}$
Pulse 41.0 degrees

Acg. time 0.800 sect

OBSERVE C13 $75451976 \mathrm{MHZ}$

DECOUPLE H1, 300.0688846 M

continuously on

Line broadening $1.5 \mathrm{H}$

Total time $3 \mathrm{hr}, 54 \mathrm{~min}, 4 \mathrm{sec}$

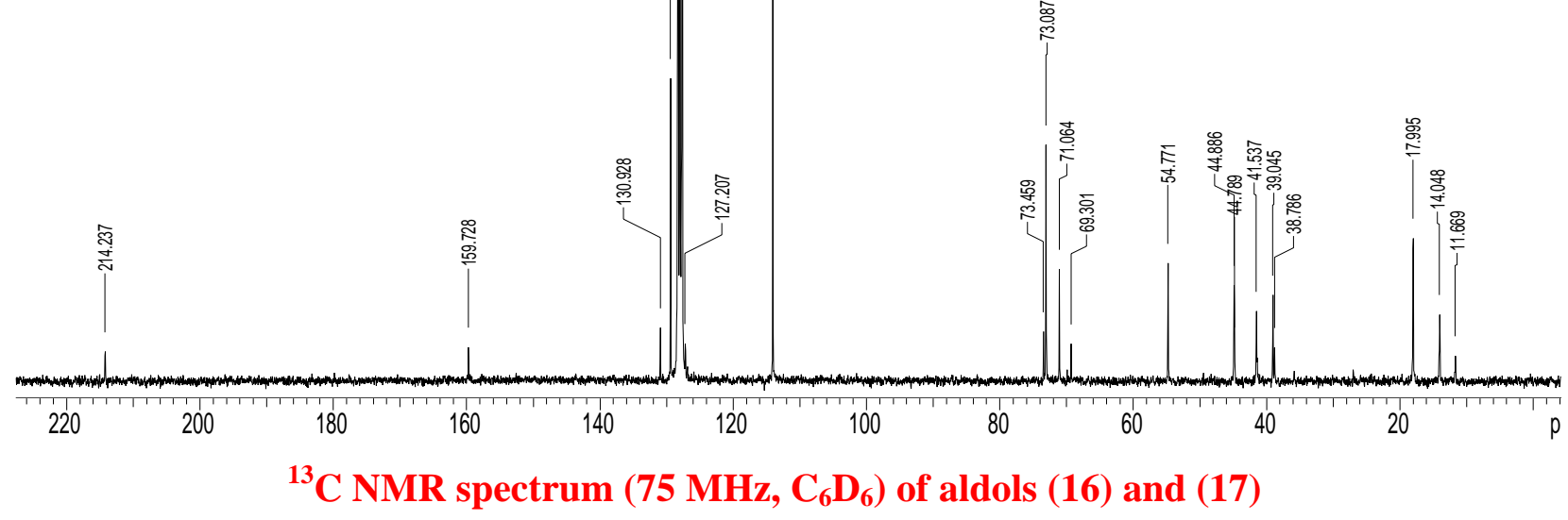




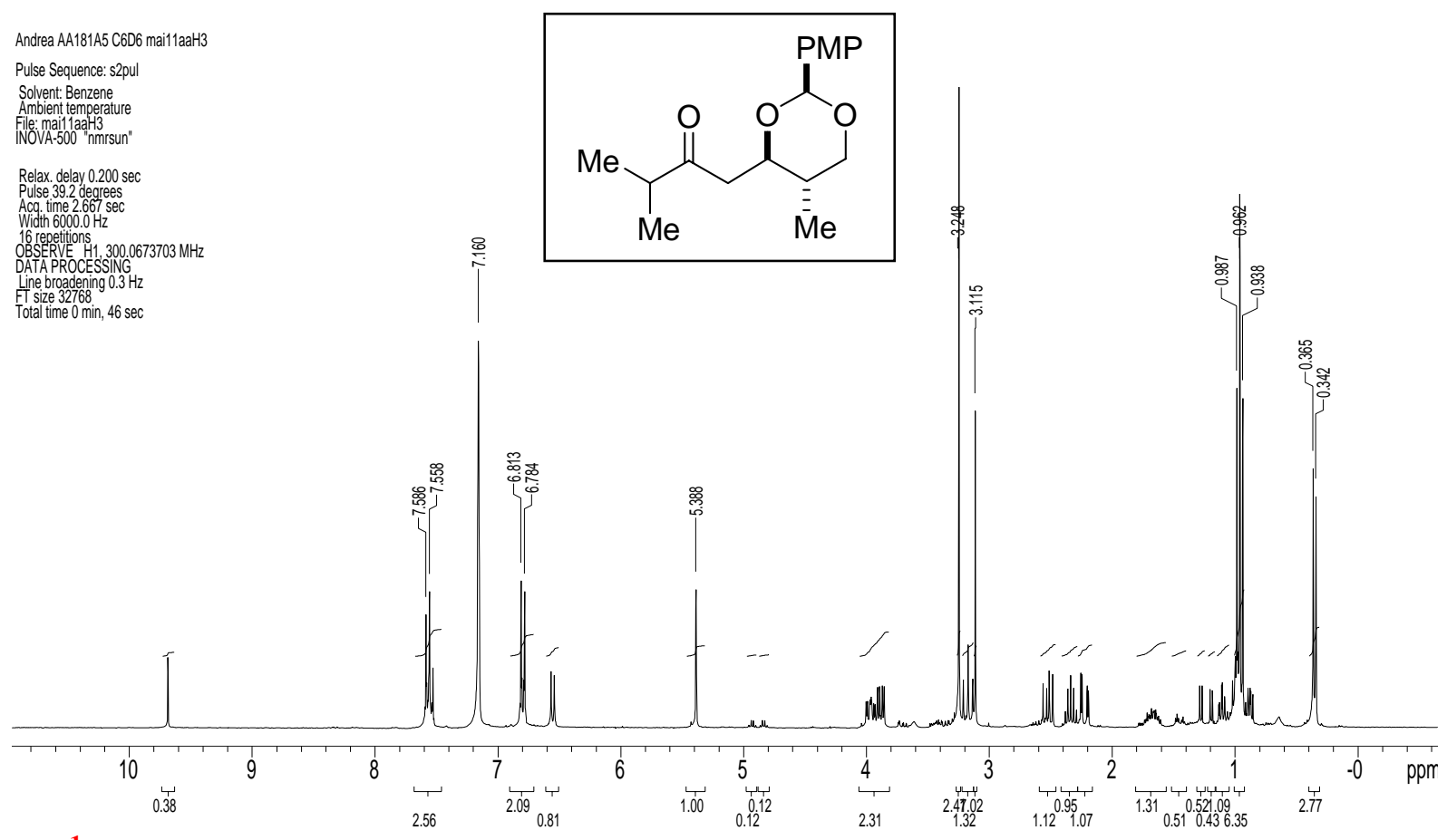

${ }^{1} \mathrm{H}$ NMR spectrum (300 MHz, $\mathrm{C}_{6} \mathrm{D}_{6}$ ) of benzylidene acetal derived from aldol (16)

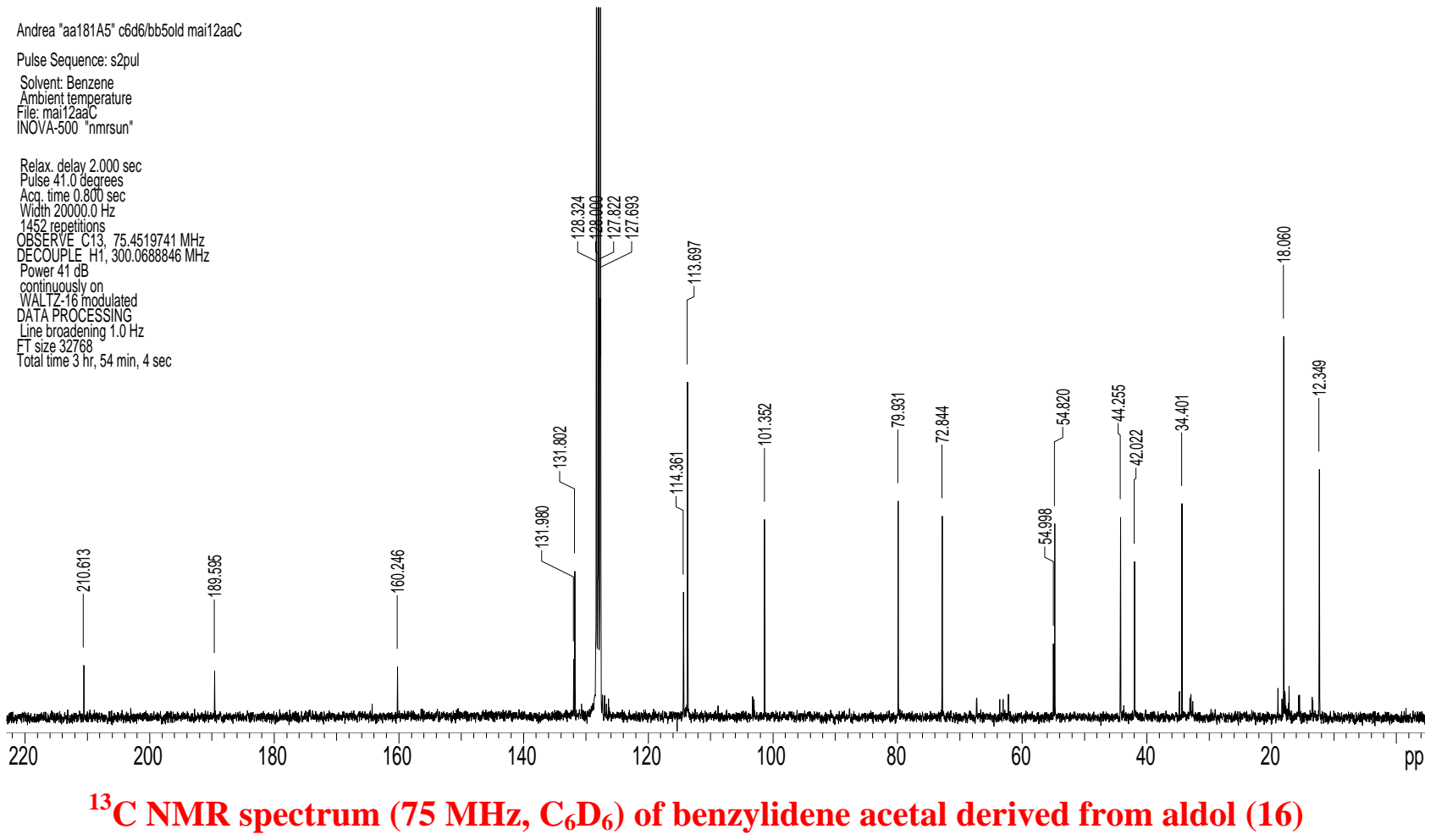




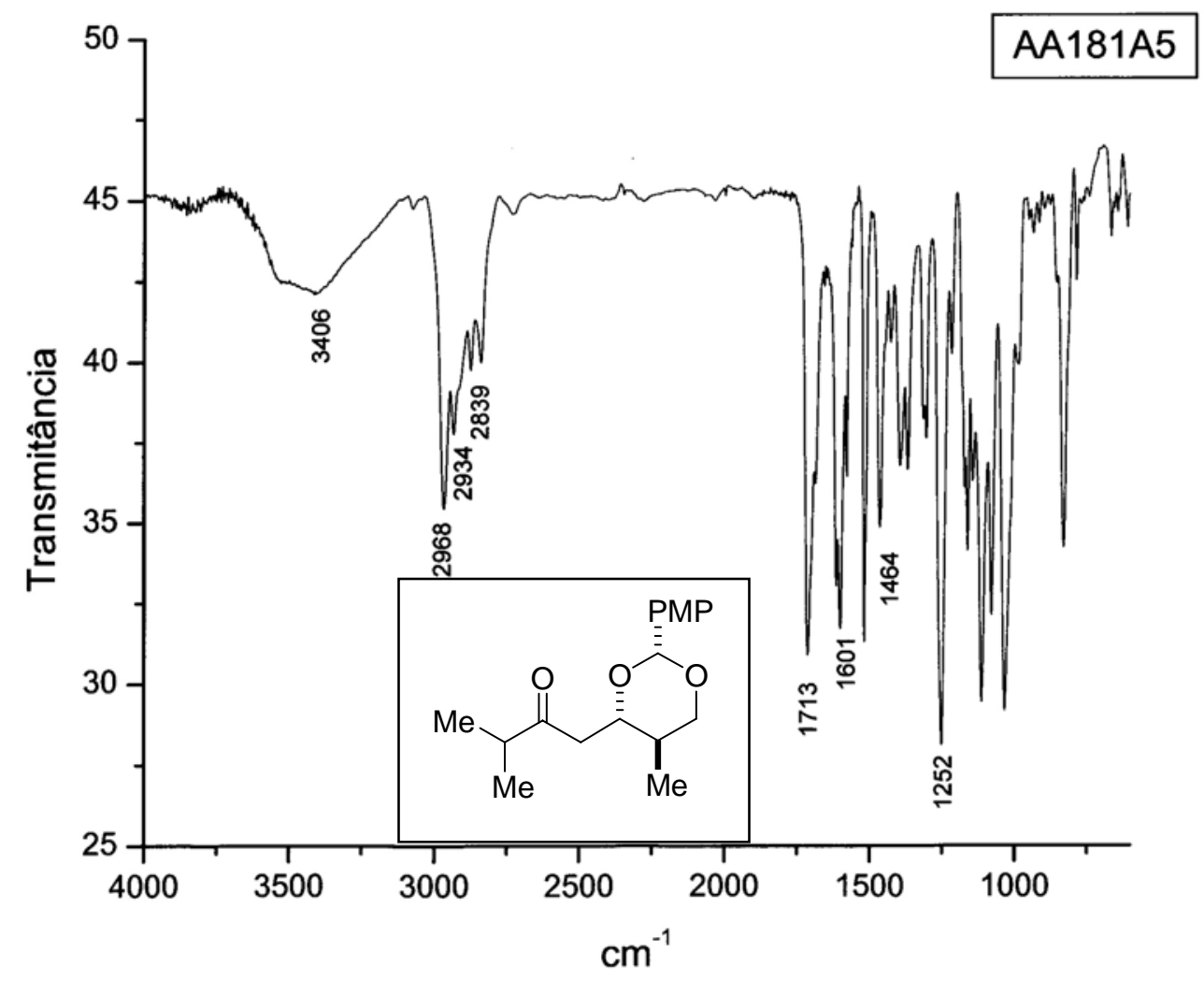

IR spectrum (film) of benzylidene acetal derived from aldol (16)

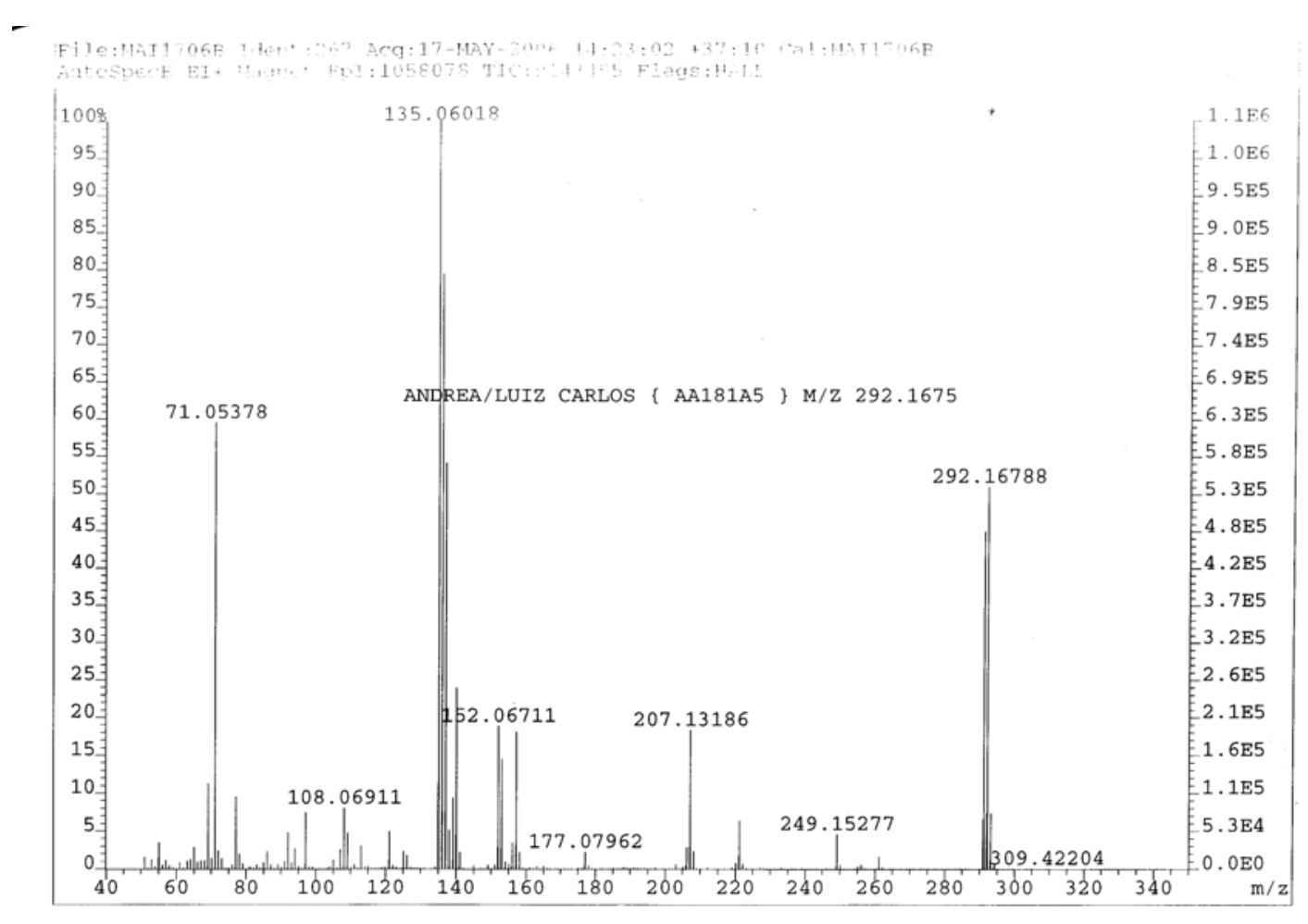

HRMS of benzylidene acetal derived from aldol (16) 


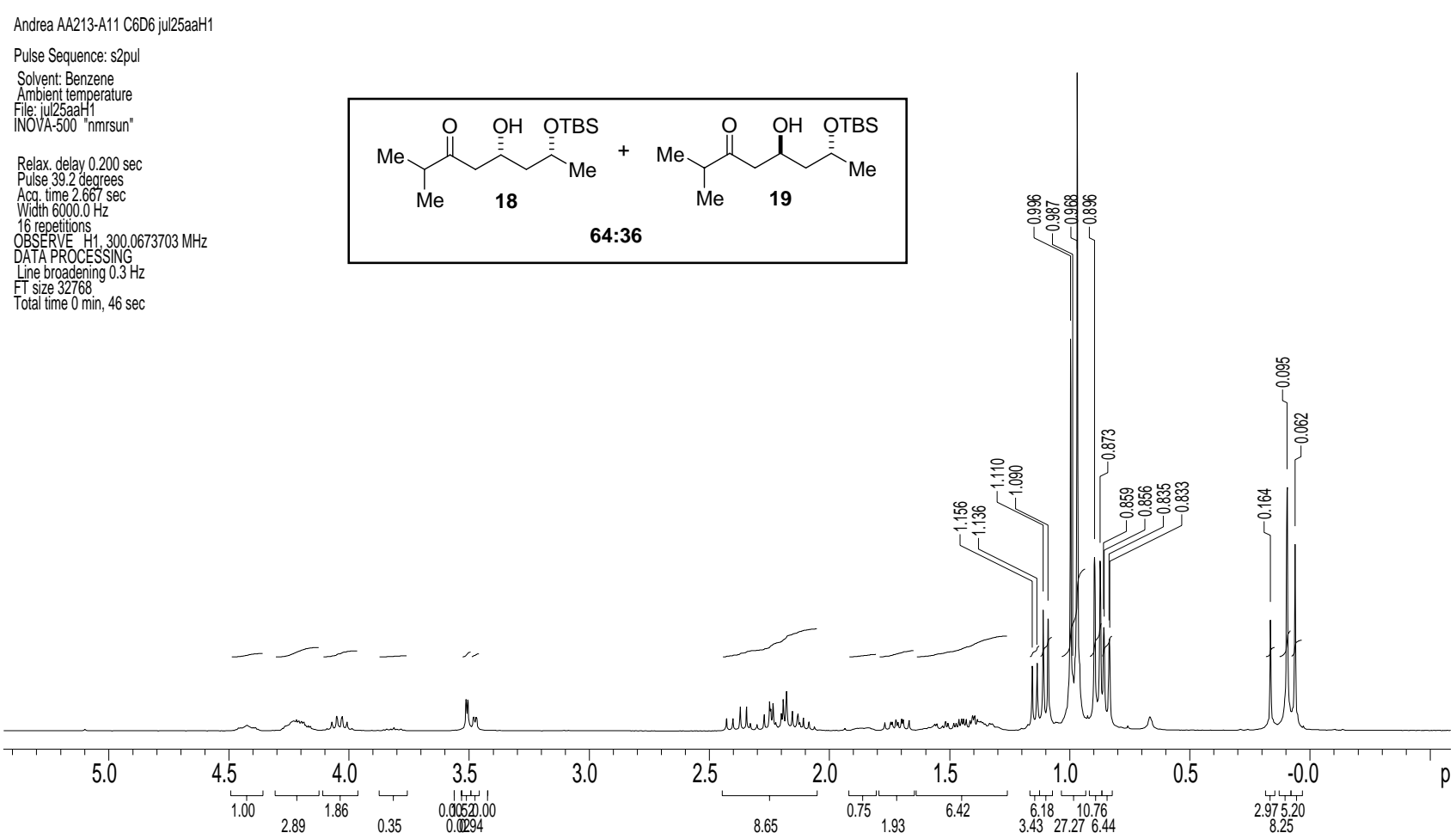

\section{${ }^{1} \mathrm{H}$ NMR spectrum (300 MHz, $\mathrm{C}_{6} \mathrm{D}_{6}$ ) of a mixture aldol 18 and 19}

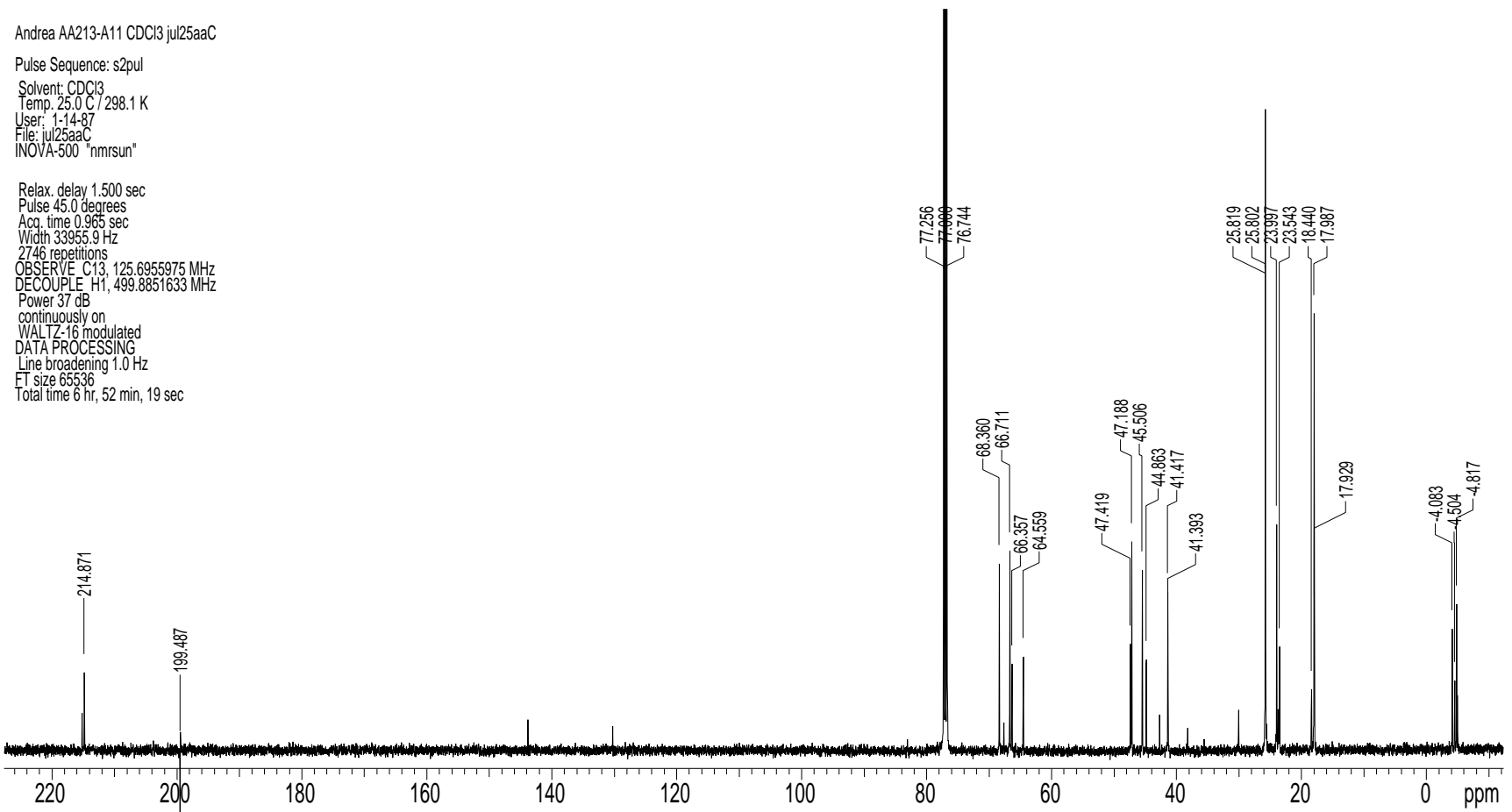

${ }^{13} \mathrm{C}$ NMR spectrum (75 MHz, $\mathrm{C}_{6} \mathrm{D}_{6}$ ) of a mixture of aldols 18 and 19 
(2R,5R,6S)-6-((4-methoxybenzyloxy)methyl)-5-hydroxy-2-((2S,4S,5S)-2-(4-methoxyphenyl)-5methyl-1,3-dioxan-4-yl)heptan-3-one (23):
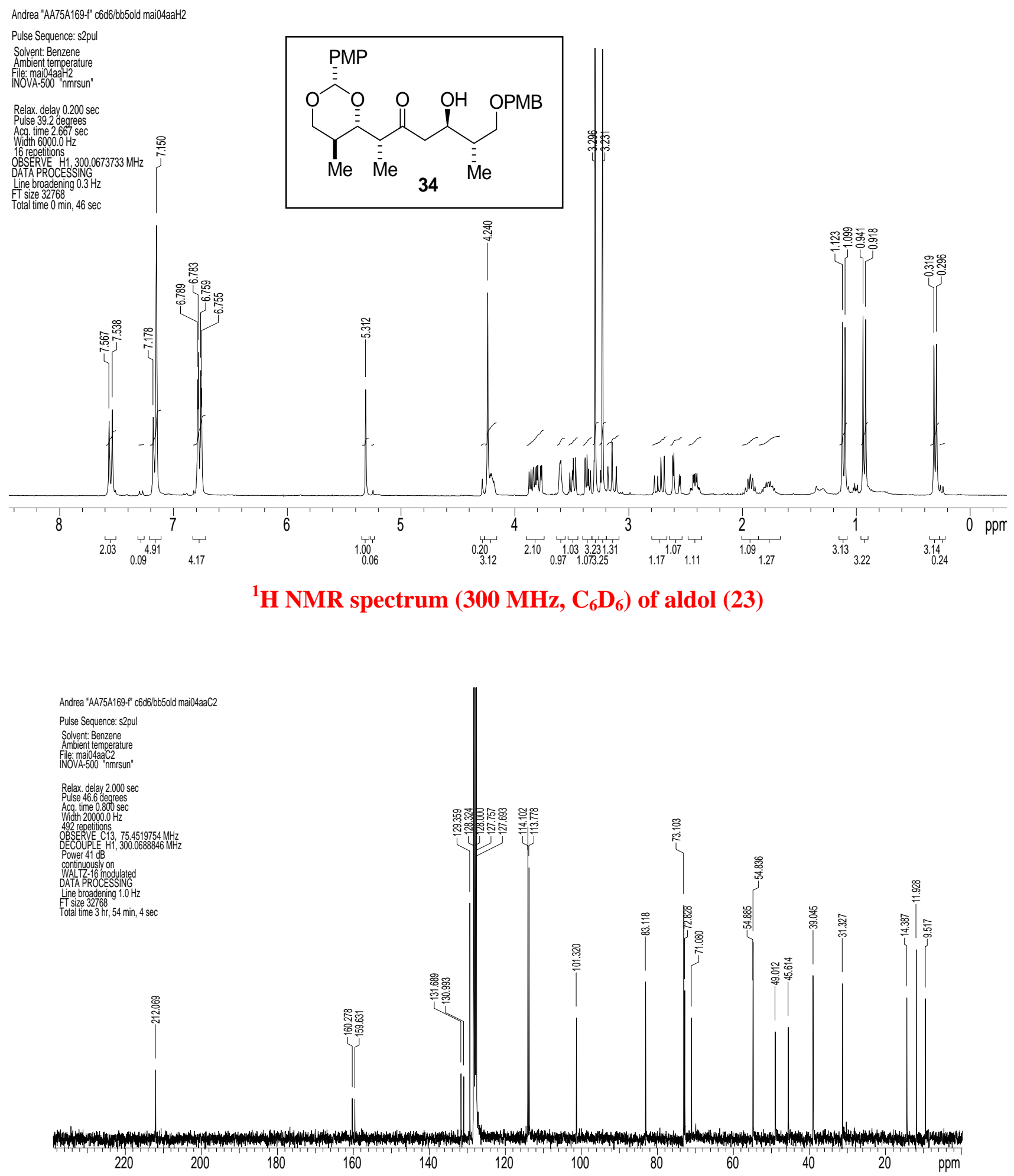

${ }^{13} \mathrm{C}$ NMR spectrum (75 $\mathrm{MHz}, \mathrm{C}_{6} \mathrm{D}_{6}$ ) of aldol (23) 

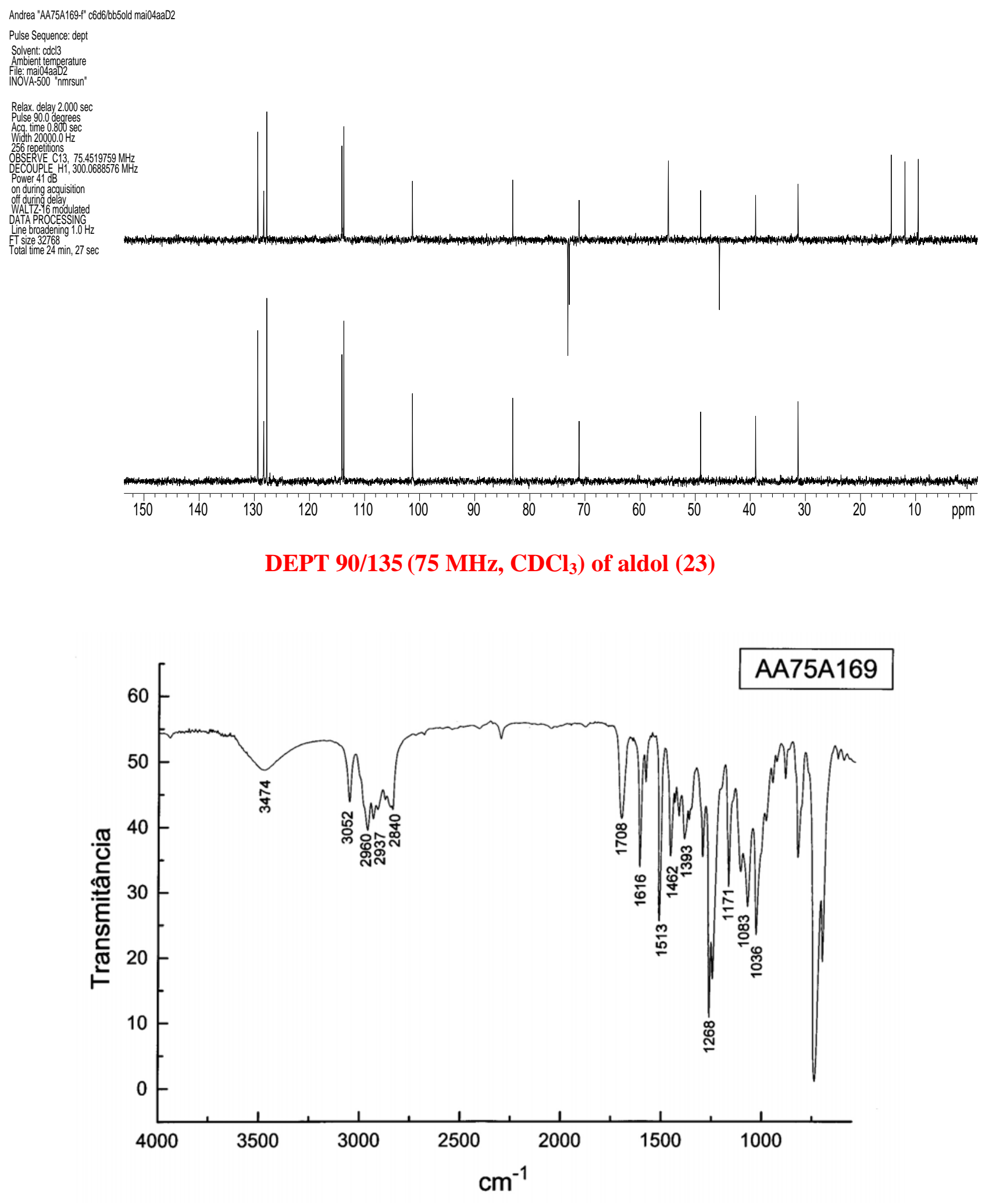

IR spectrum (film) of aldol (23) 


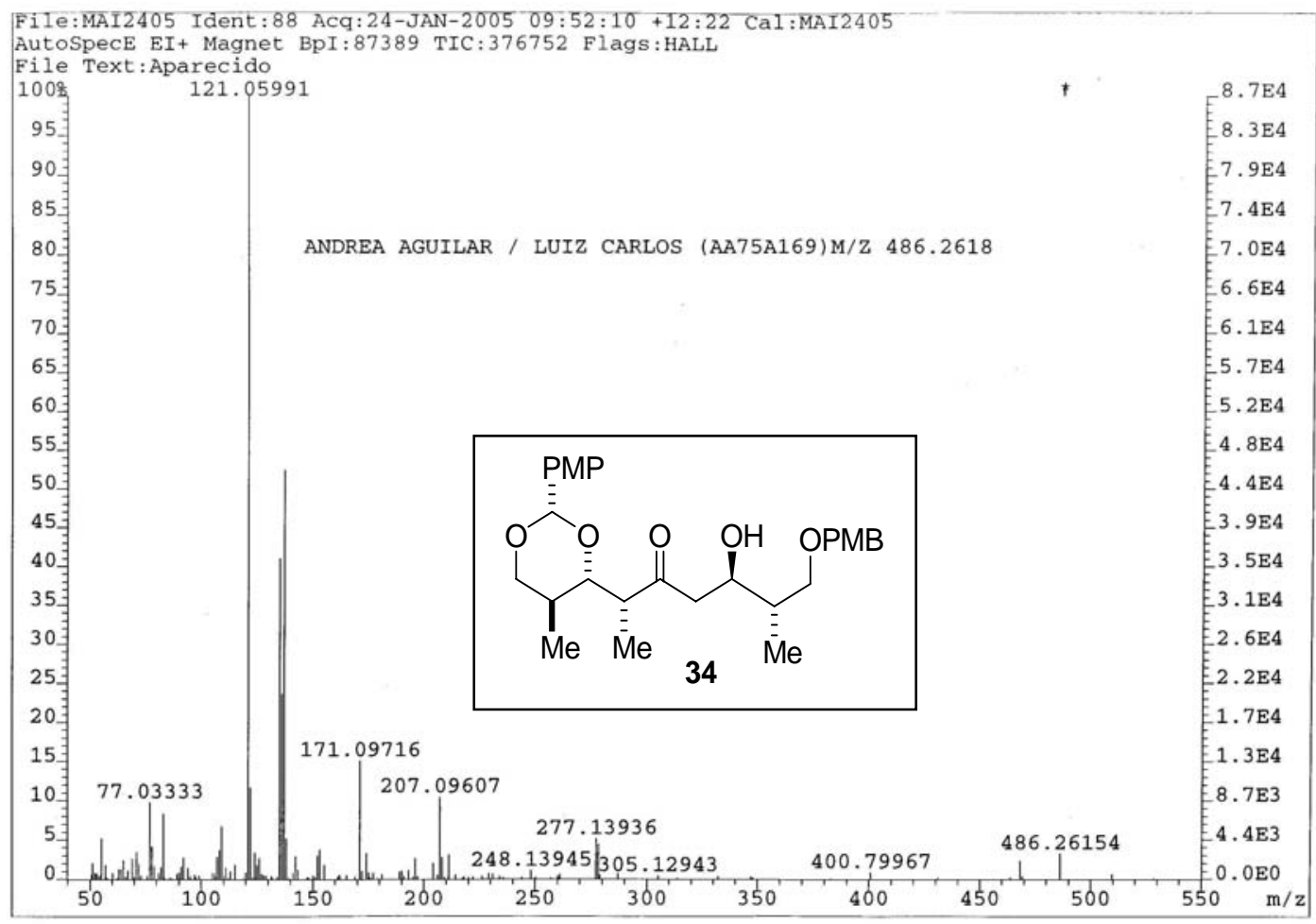

HRMS of aldol (23)

(2R,5R,6S)-6-((benzyloxy)methyl)-5-hydroxy-2-((2S,4S,5S)-2-(4-methoxyphenyl)-5-methyl-1,3dioxan-4-yl)heptan-3-one (24):

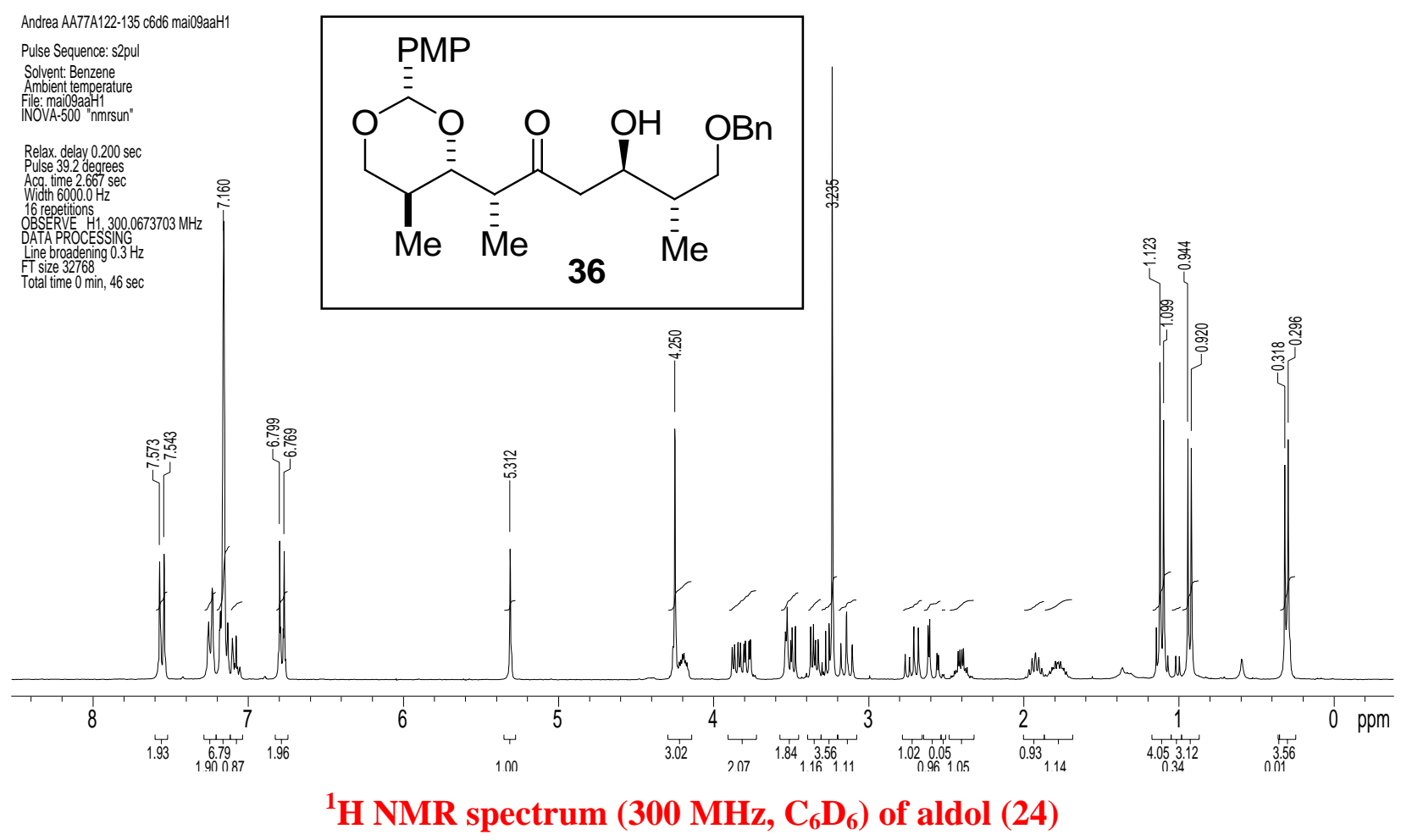


andrea aa77a122 cdcl3 mai03aaC

Pulse Sequence: s2pul

Solvent: $\mathrm{CDCl} 3$

Ambient temperature
File: mai03aaC

File: mai03aaC
INOVA-500 "nmrsun"

Relax. delay $2.000 \mathrm{sec}$

Acc. time 0.800 sec

Width $20000.0 \mathrm{~Hz}$

501 repetitions $75152029 \mathrm{MHz}$

DECOUPLE H1, $300.0688576 \mathrm{MHz}$
Power $41 \mathrm{~dB}$

Power $41 \mathrm{~dB}$
continuously on

WALTZ-16 modulated

DATA PROCESSING

Line broadening $1.0 \mathrm{~Hz}$

Total time $7 \mathrm{hr}, 48 \mathrm{~min}, 8 \mathrm{sec}$

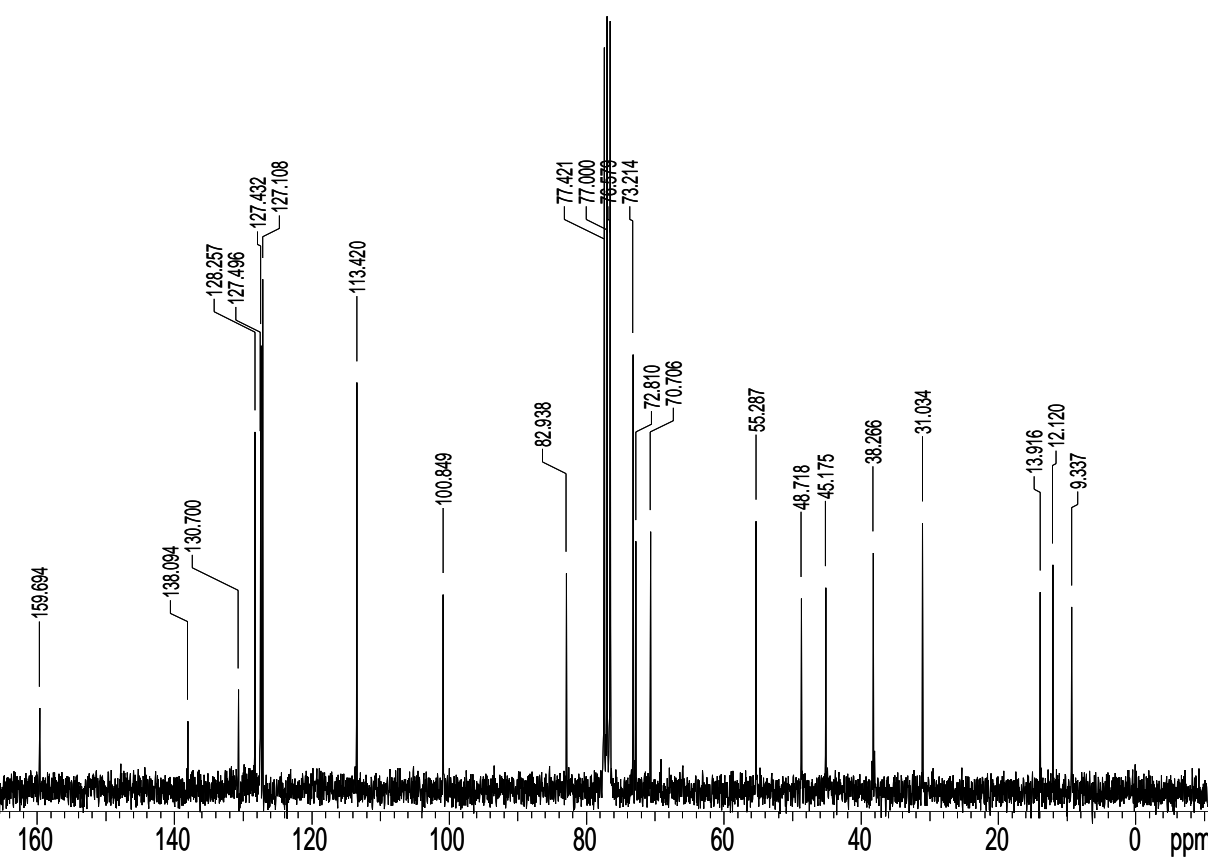

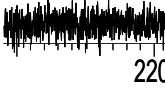

200

180

160

140

120

100

80

60

pprr

${ }^{13} \mathrm{C}$ NMR spectrum (75 $\mathrm{MHz}, \mathrm{CDCl}_{3}$ ) of aldol (24)

Pulse Sequence: dept

Solvent: cdcl3
Ambient temperature

File: maiogaaD1
INOVA-500 "nmrsun"

Relax. delay $2.000 \mathrm{sec}$

Pulse 90.0 degrees

Acg time $200000 \mathrm{~Hz}$

OBSERVE C13, $75.4519746 \mathrm{MHz}$
DECOUPLE H1, $300.0688576 \mathrm{MHz}$

Power $41 \mathrm{~dB}$

on during acquisition

WAUTZ.16 modulated

DATA PROCESSING

Line broadening $1.0 \mathrm{~Hz}$

FT size 32768
Total time $24 \mathrm{~min}, 27 \mathrm{sec}$
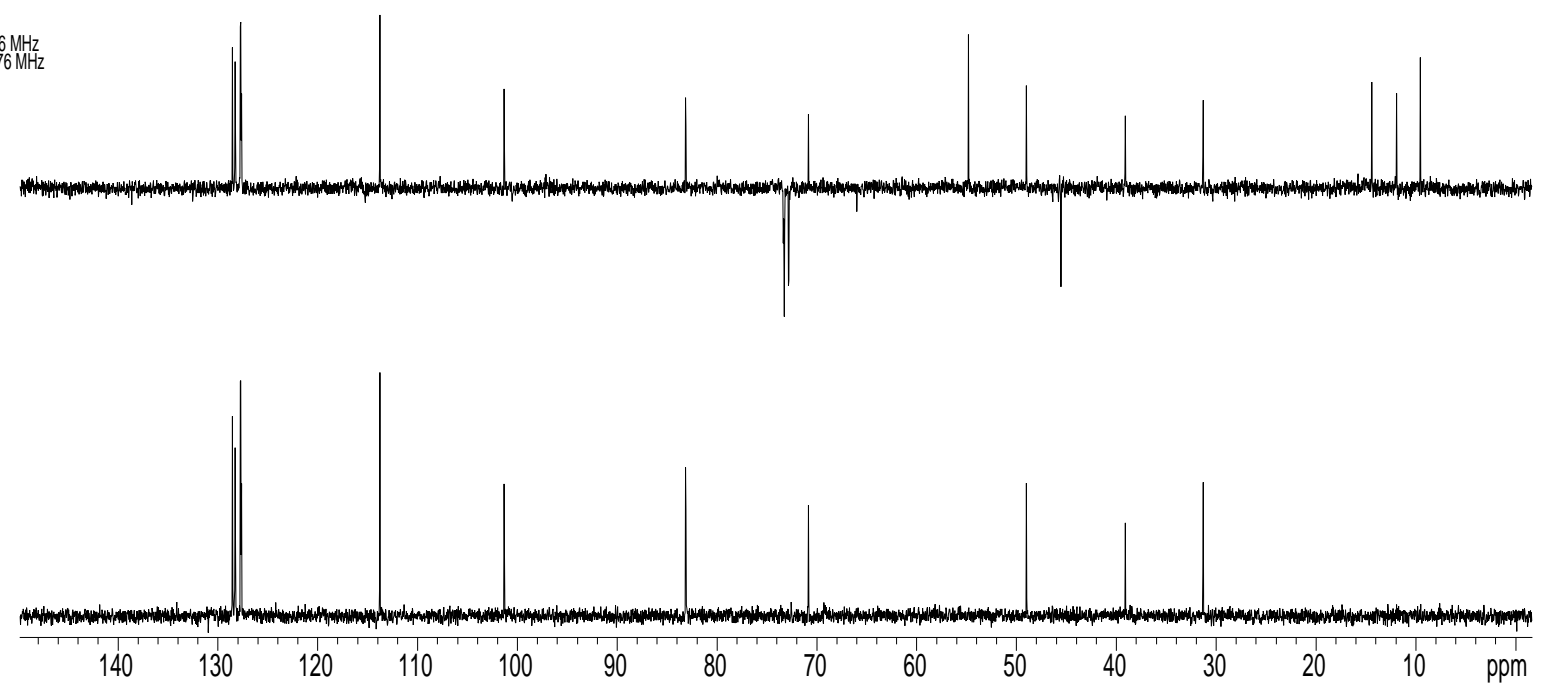

DEPT 90/135 (75 MHz, CDCl 3 ) of aldol (24) 


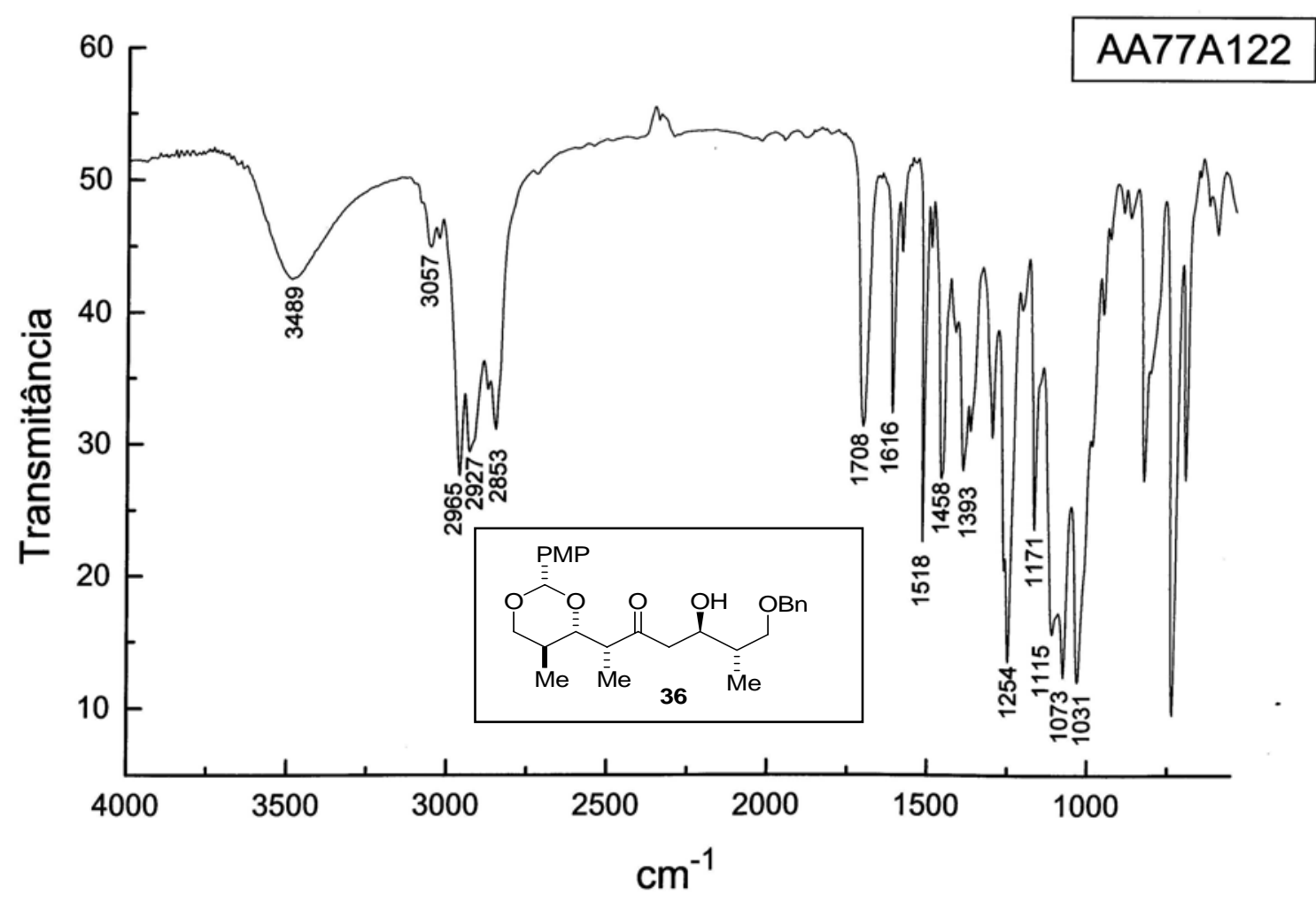

IR spectrum (film) of aldol (24)

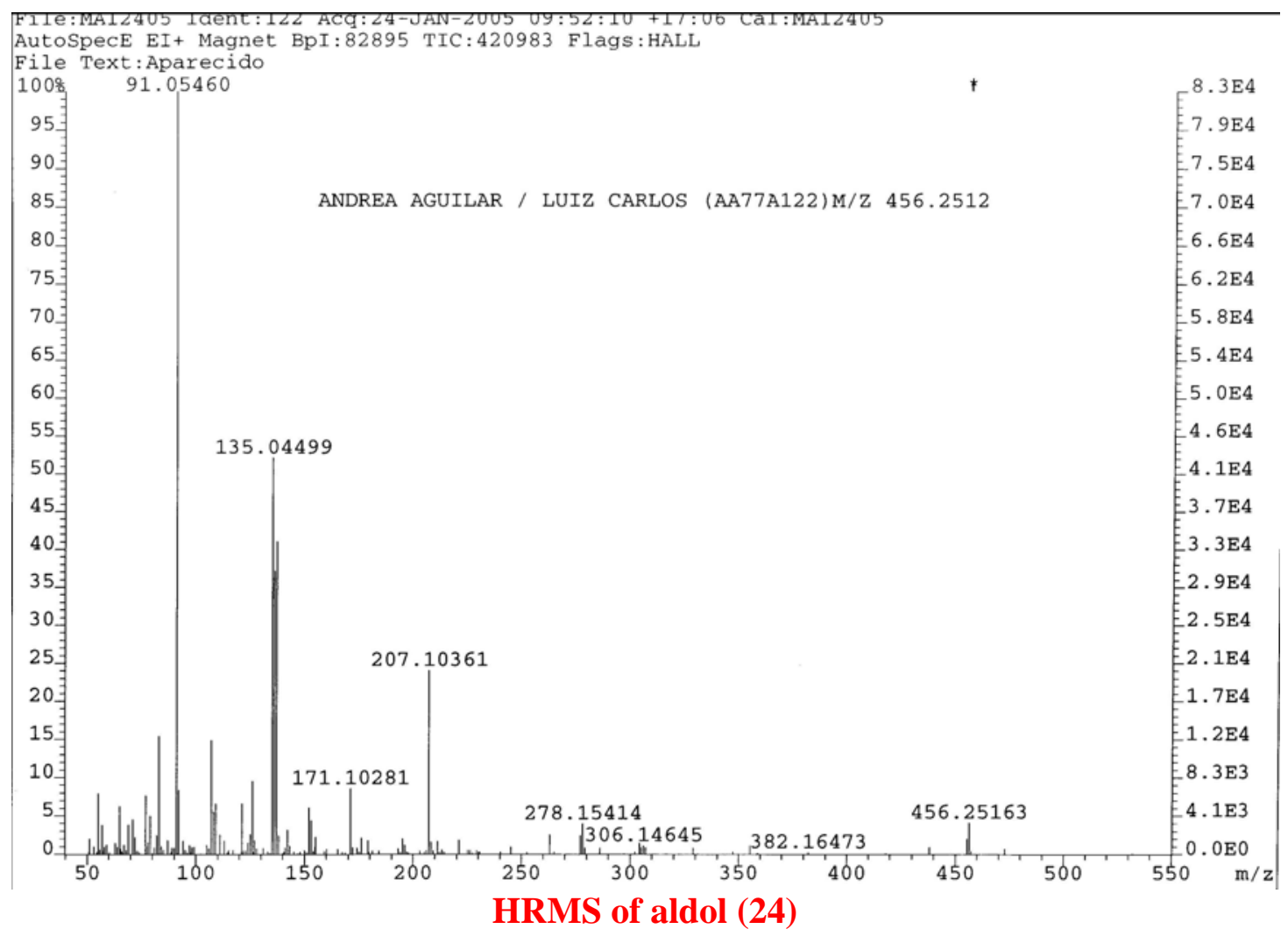


(R)-1-((2R,4R,5S)-2-(4-methoxyphenyl)-5-methyl-1,3-dioxan-4-yl)-3-((2S,4S,5S)-2-(4methoxyphenyl)-5-methyl-1,3-dioxan-4-yl)butan-2-one (25):
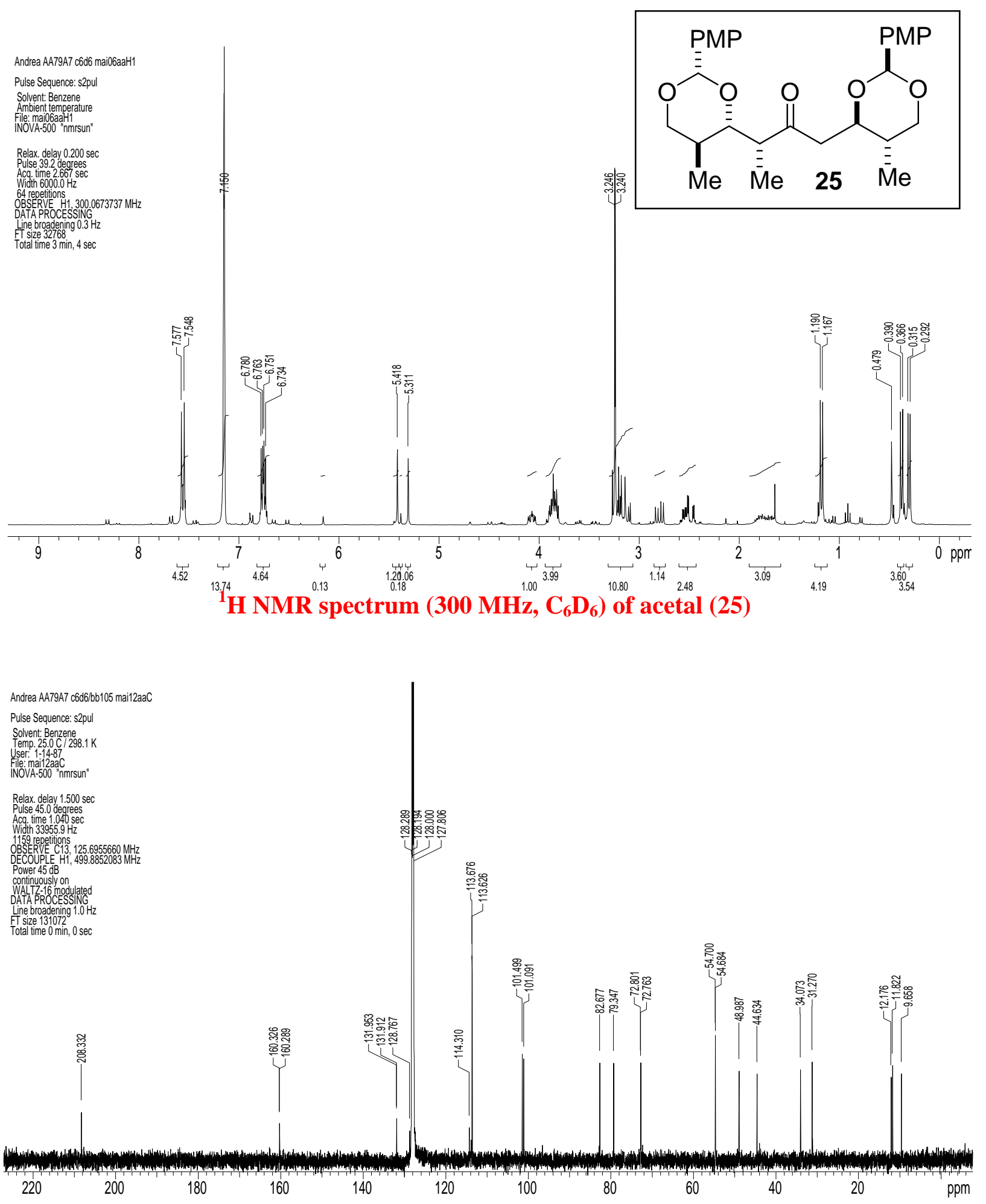

${ }^{13} \mathrm{C}$ NMR spectrum (125 MHz, $\mathrm{C}_{6} \mathrm{D}_{6}$ ) of acetal (25) 
Andrea AA79A7 c6d6/bb105 mai12aaD

Pulse Sequence: dept

Solvent: $\mathrm{cdc} / 3$
Temp. $25.0 \mathrm{C} / 298.1 \mathrm{~K}$

INOVA-500 "nmrsun"

Relax. delay 1.500 sec
Pulse 900 dearees

Acg. time $1.040 \mathrm{sec}$

Width $33955.9 \mathrm{~Hz}$

OBS repetitions

DECOUPLE H1, $499.8851633 \mathrm{MHz}$

Power $45 \mathrm{~dB}$

on during acquisition

WALTZ-16 modulated

Line broadening $1.0 \mathrm{~Hz}$

FT size 131072
Total time $44 \mathrm{~min}, 4 \mathrm{sec}$
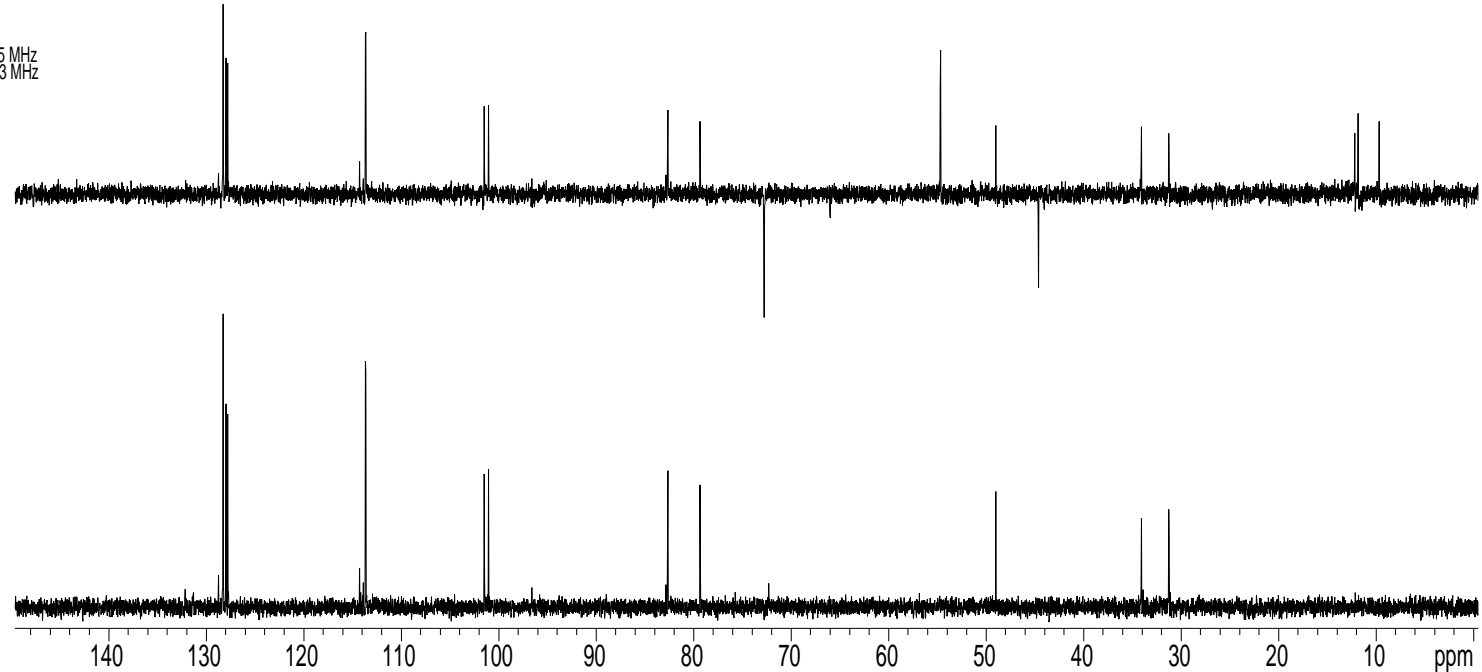

DEPT 90/135 (125 MHz, $\left.\mathrm{C}_{6} \mathrm{D}_{6}\right)$ of acetal (25)

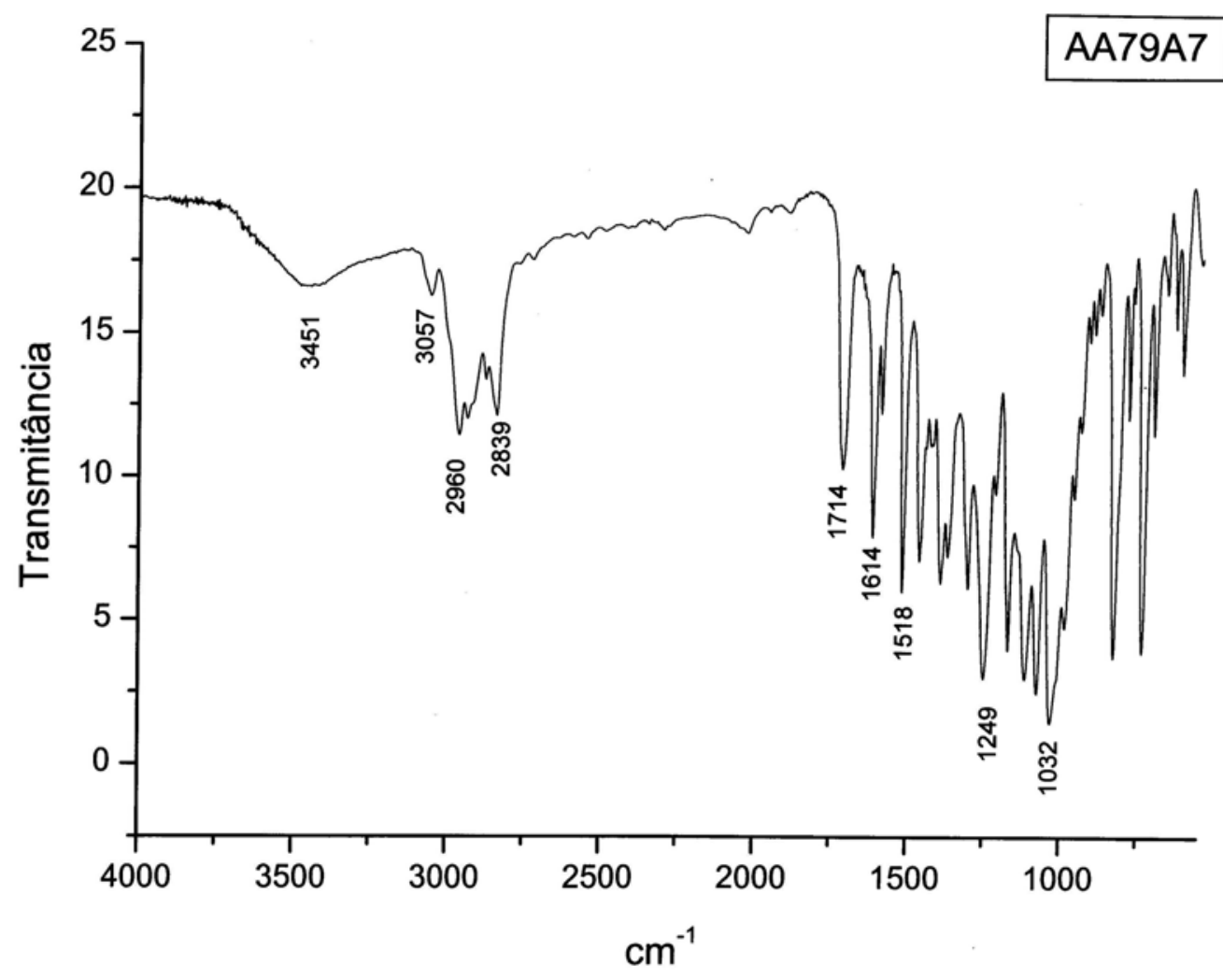

IR spectrum (film) of acetal (25) 


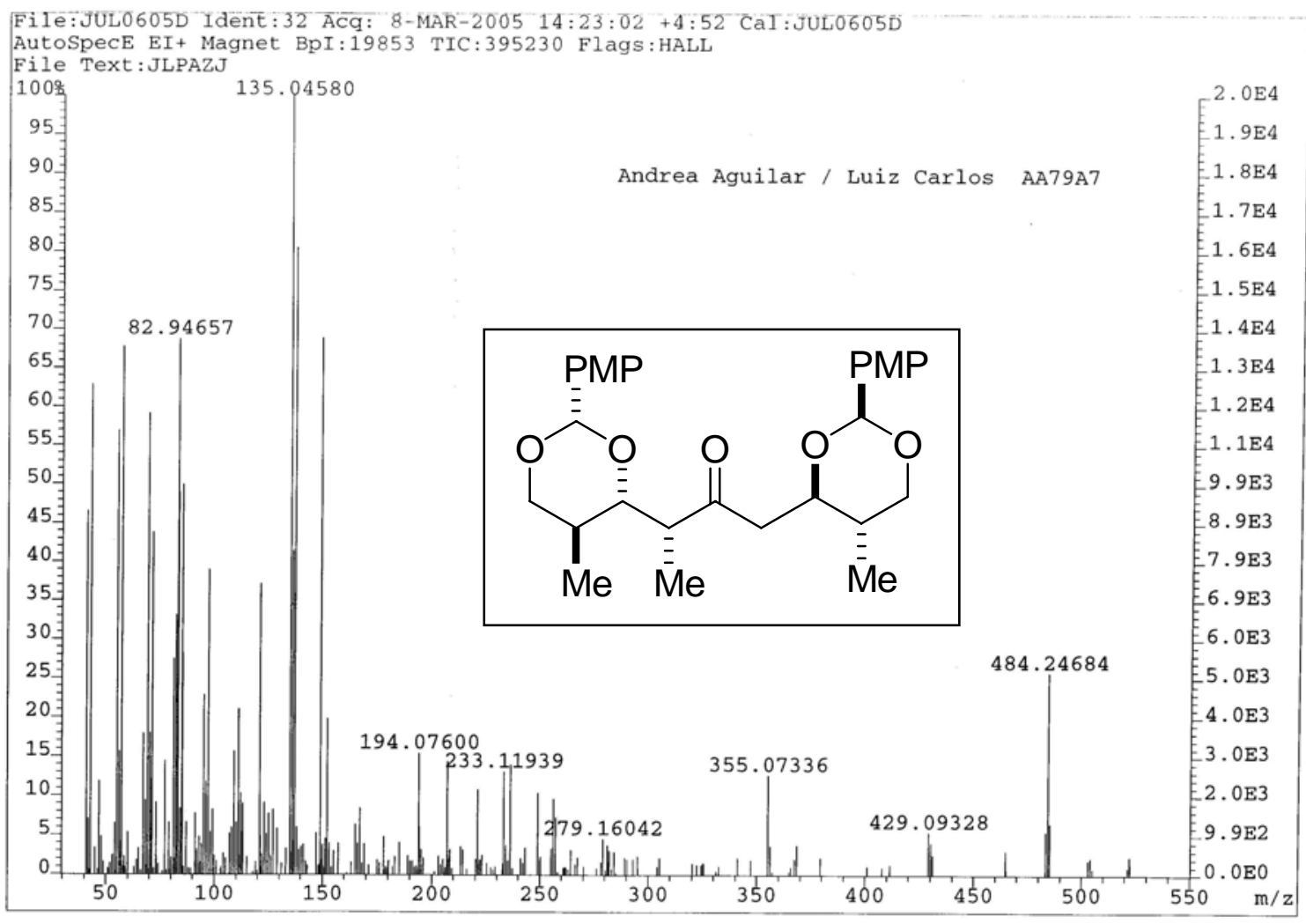

HRMS of acetal (25) 
(2R,5R,6R)-7-(4-methoxybenzyloxy)-5-hydroxy-2-((2S,4S,5S)-2-(4-methoxyphenyl)-5-methyl-

\section{1,3-dioxan-4-yl)-6-methylheptan-3-one (26):}
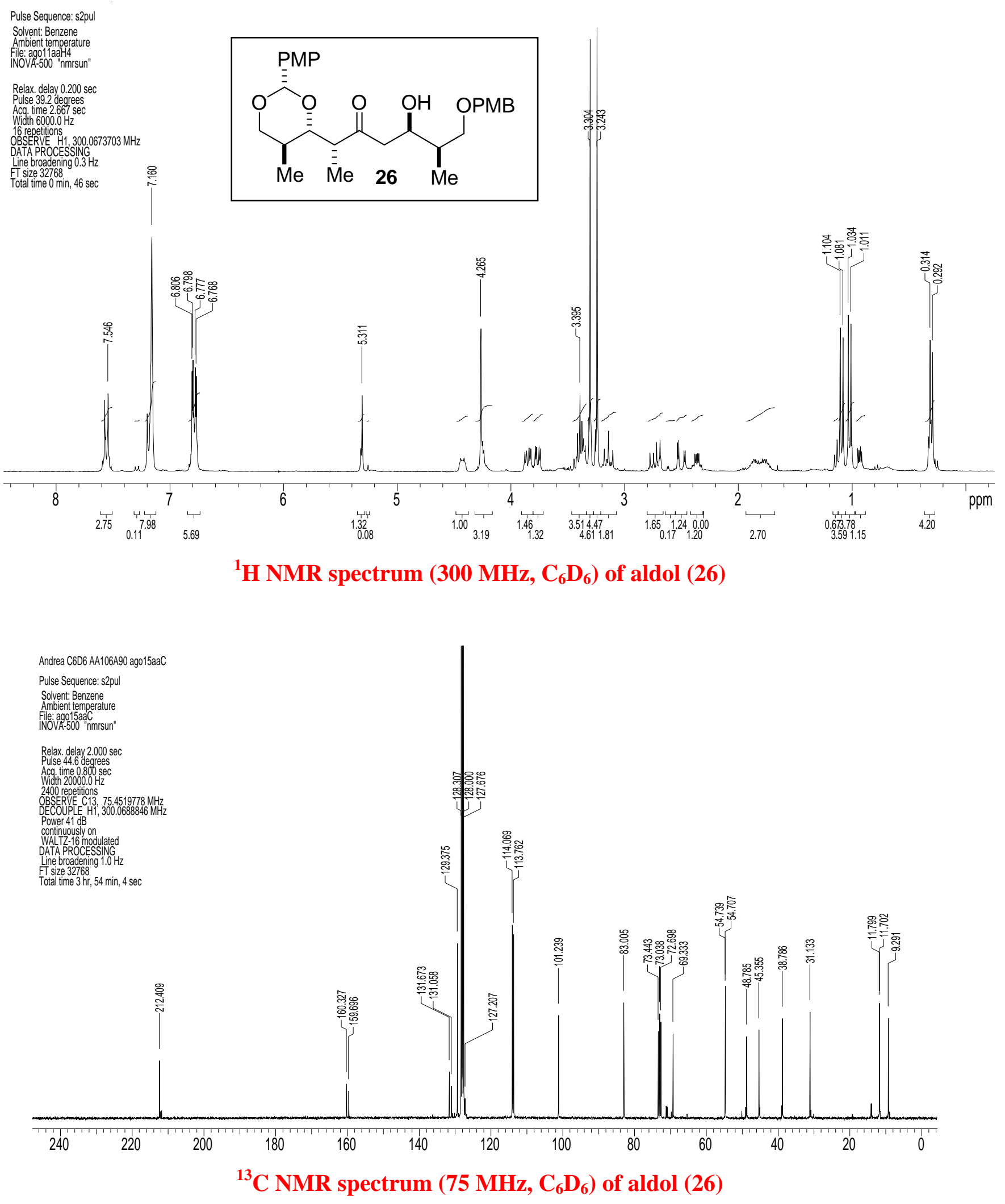

${ }^{1} \mathrm{H}$ NMR spectrum (300 MHz, $\mathrm{C}_{6} \mathrm{D}_{6}$ ) of aldol (26) 
Pulse Sequence: dept

Solvent: cdcl3

Ambient temperature

File: ago $13 a$ ad
INOVA-500 "nmrsun"

Relax. delay $2.000 \mathrm{sec}$

Pulse 90.0 degrees
Acq. time $0.800 \mathrm{sec}$

Acq. time $0.800 \mathrm{sec}$

Whath $2000.0 \mathrm{~Hz}$

3200 repetitions
OBSERVE C C13, $75.4519759 \mathrm{MHz}$
DECOUPLE H1', $300.0688576 \mathrm{MHz}$

Power $41 \mathrm{~dB}$

on during acquisition

WALTZ-16 modulated

DATA PROCESSING

Total time 5 hr, $1 \mathrm{~min}, 28 \mathrm{sec}$
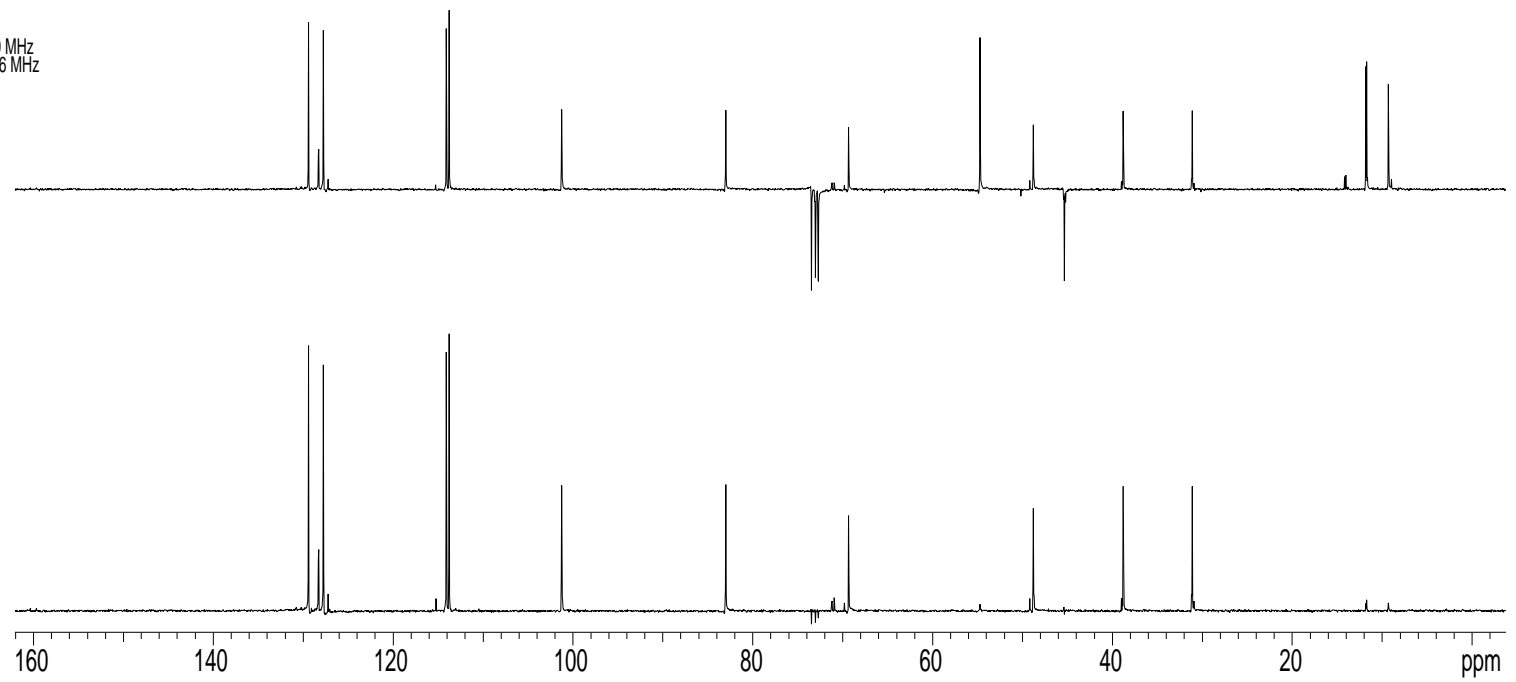

DEPT 90/135 (75 MHz, $\mathrm{C}_{6} \mathrm{D}_{6}$ ) of aldol (26)

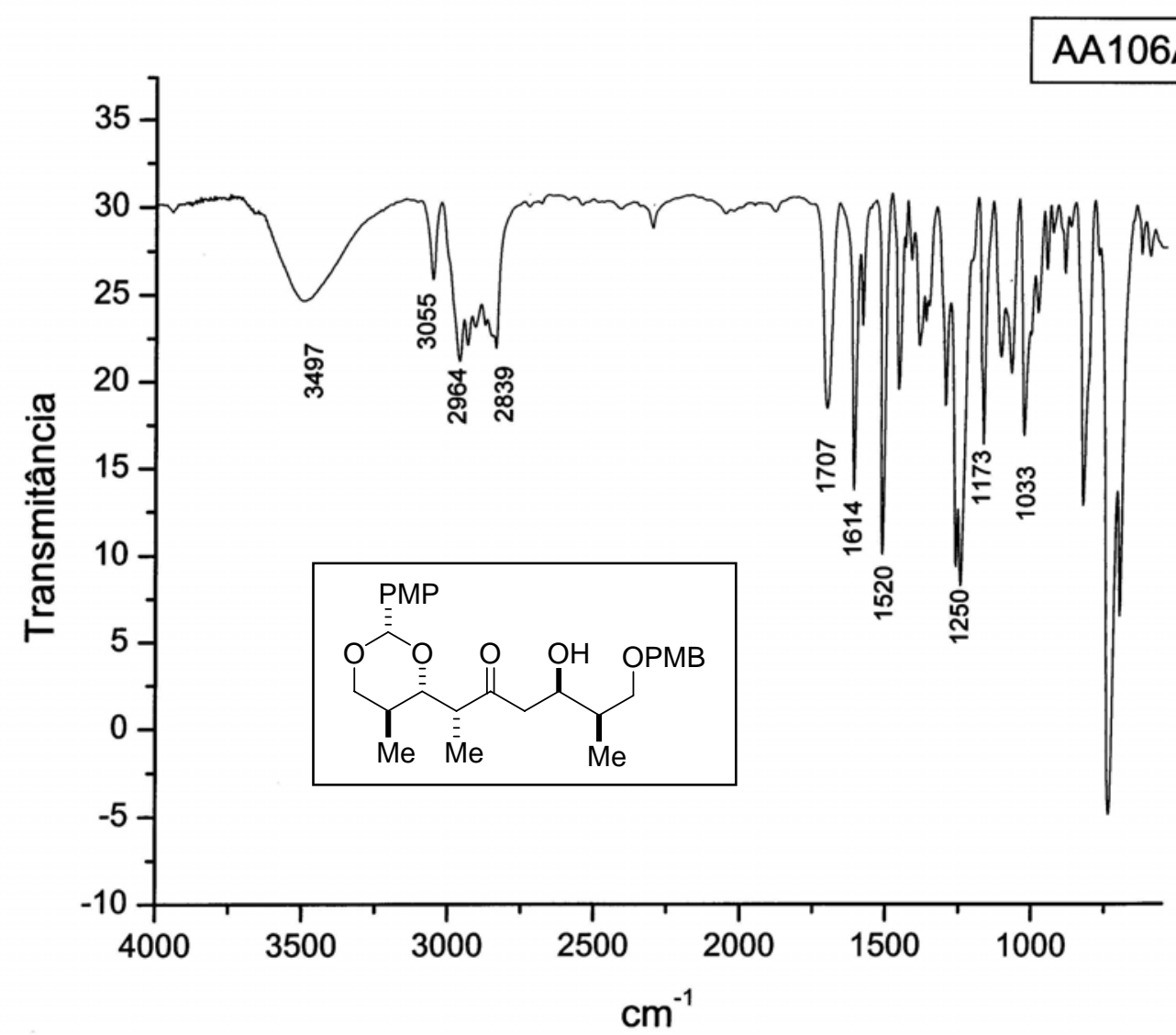

IR spectrum of aldol (26) 


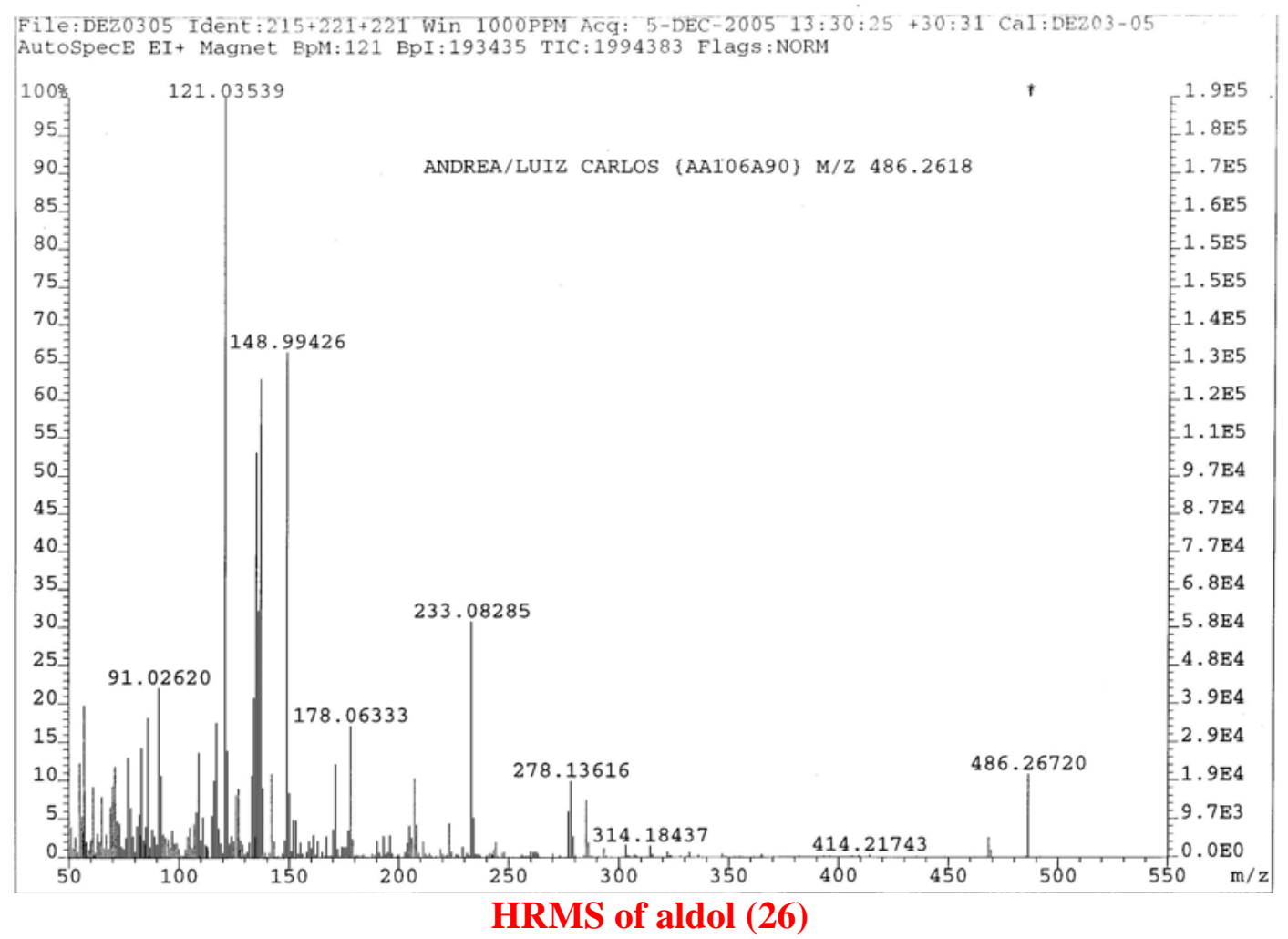

(R)-1-((2S,4R,5R)-2-(4-methoxyphenyl)-5-methyl-1,3-dioxan-4-yl)-3-((2S,4S,5S)-2-(4methoxyphenyl)-5-methyl-1,3-dioxan-4-yl)butan-2-one (27):

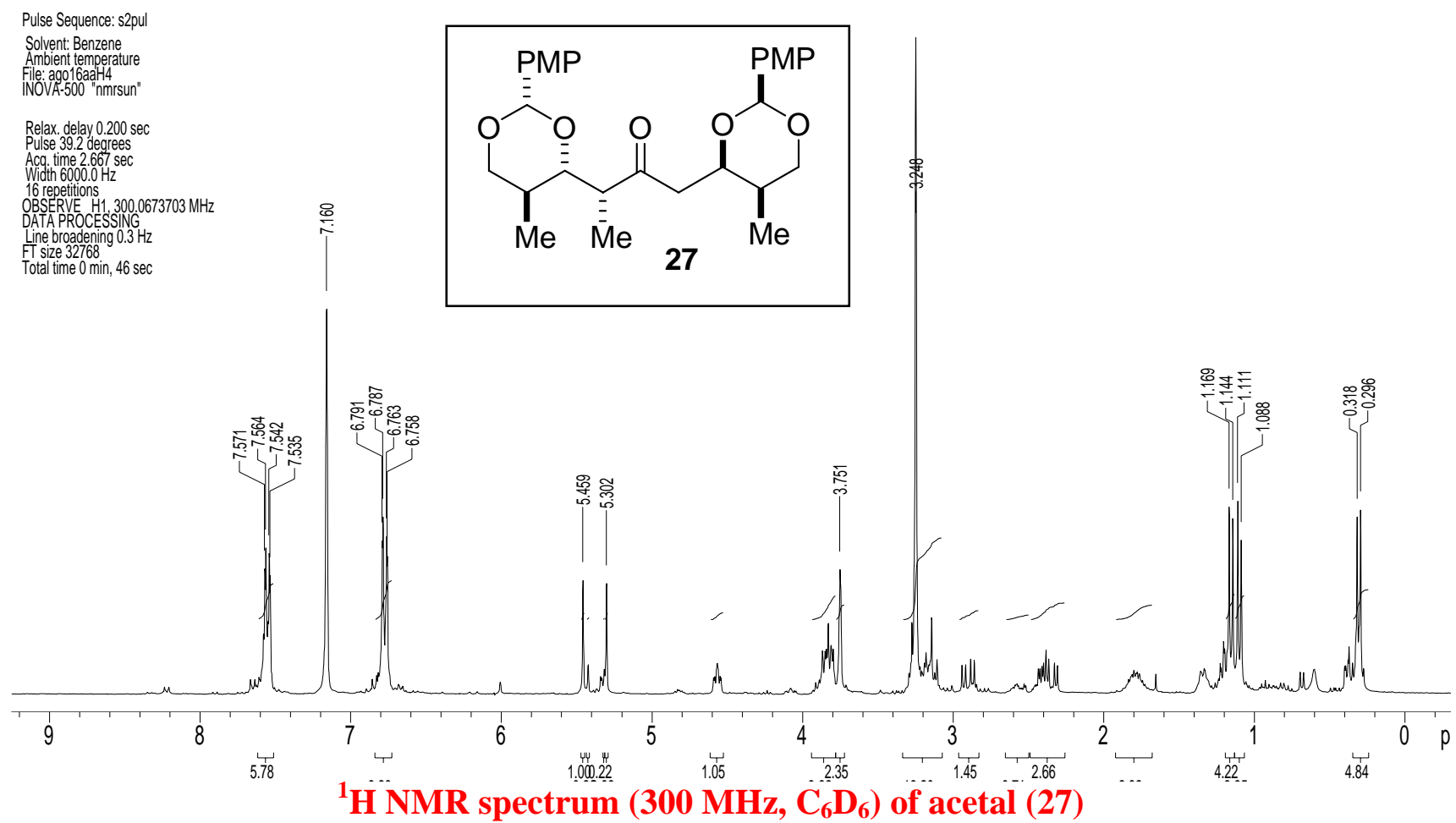


Andrea AA107A4 c6d6 ago18aaC

Pulse Sequence: s2pul

Solvent: $\mathrm{CDCl} 3$

Ambient temperature

File: 2 ago $18 \mathrm{a} a \mathrm{C}$

File: ago18aaC
INOVA-500 "nmrsun"

Relax. delay $1.500 \mathrm{sec}$

Acq. time $1.040 \mathrm{sec}$

Width $33955.9 \mathrm{~Hz}$

1680 repetitions
OBSERVE C13,125.6955666 MHz

OBSERVE C13, $125.6955666 \mathrm{MHz}$
DECOUPLE H1, $499.8851633 \mathrm{MHz}$

Power $45 \mathrm{~dB}$

continuously on

WALTZ-16 modulated
DATA PROCESSING

Line broadening $1.0 \mathrm{~Hz}$

Total time $3 \mathrm{hr}, 32 \mathrm{~min}, 24 \mathrm{sec}$

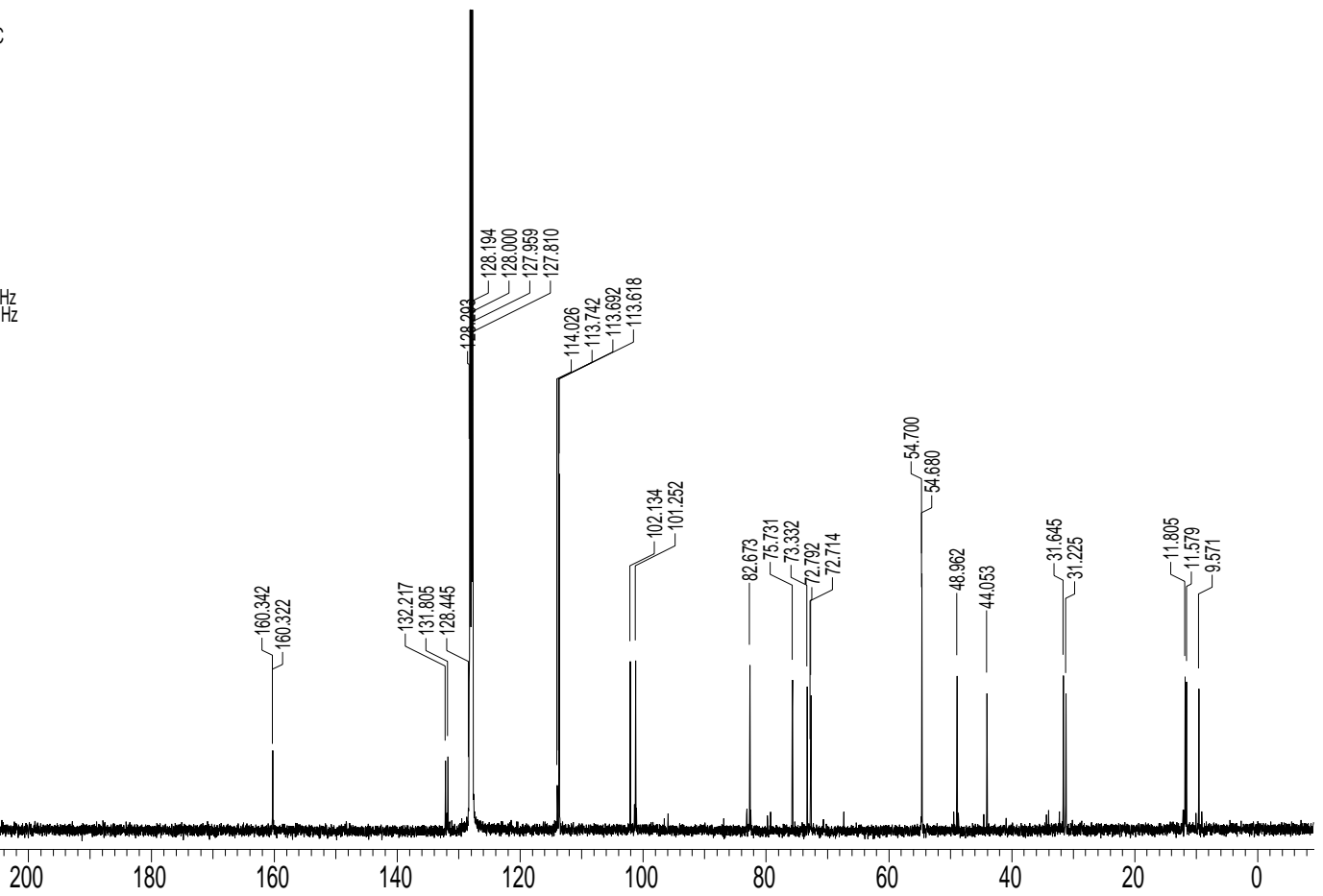

${ }^{13} \mathrm{C}$ NMR spectrum (125 MHz, $\mathrm{C}_{6} \mathrm{D}_{6}$ ) of acetal (27)

andrea c6d6 AA107A4 ago18aaD

Pulse Sequence: dept

Solvent: cdcl3

File: ago18aaD
INOVA-500 "nmrsun"

Relax. delay $2.000 \mathrm{sec}$

Pulse 90.0 degrees
Aca time $0.800 \mathrm{sec}$

Acq. time $0.800 \mathrm{sec}$
Width $20000.0 \mathrm{~Hz}$

512 repetitions
OBSERVE C13, $75.4519777 \mathrm{MHz}$
DECOUPLE H1, $300.0688576 \mathrm{MHz}$

Power $41 \mathrm{~dB}$

on during acquisition

WALTZ.16 maduled

DATA PROCESSING

Line broadening $1.0 \mathrm{~Hz}$

FT size 32768
Total time 48 min, 33 sec

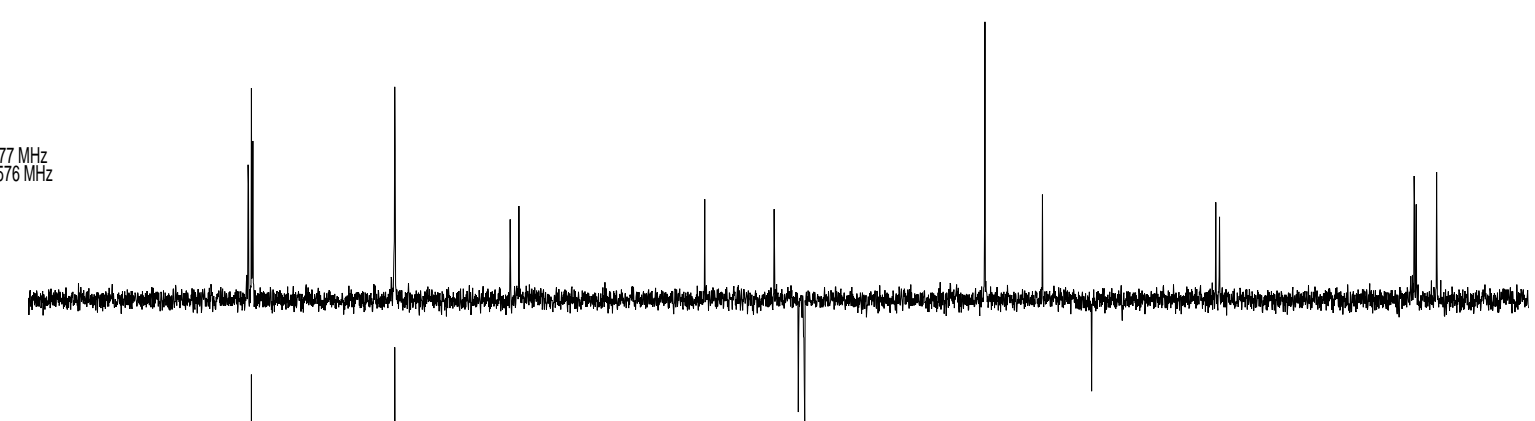

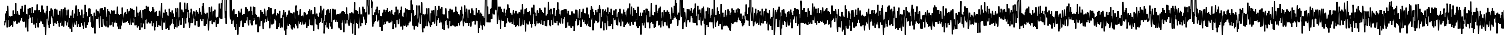
$\begin{array}{llllllllllllllllllllll}150 & 140 & 130 & 120 & 110 & 100 & 90 & 80 & 70 & 60 & 50 & 40 & 30 & 20 & 10 & \mathrm{ppm}\end{array}$

DEPT 90/135 (125 MHz, $\left.C_{6} D_{6}\right)$ of acetal (27) 

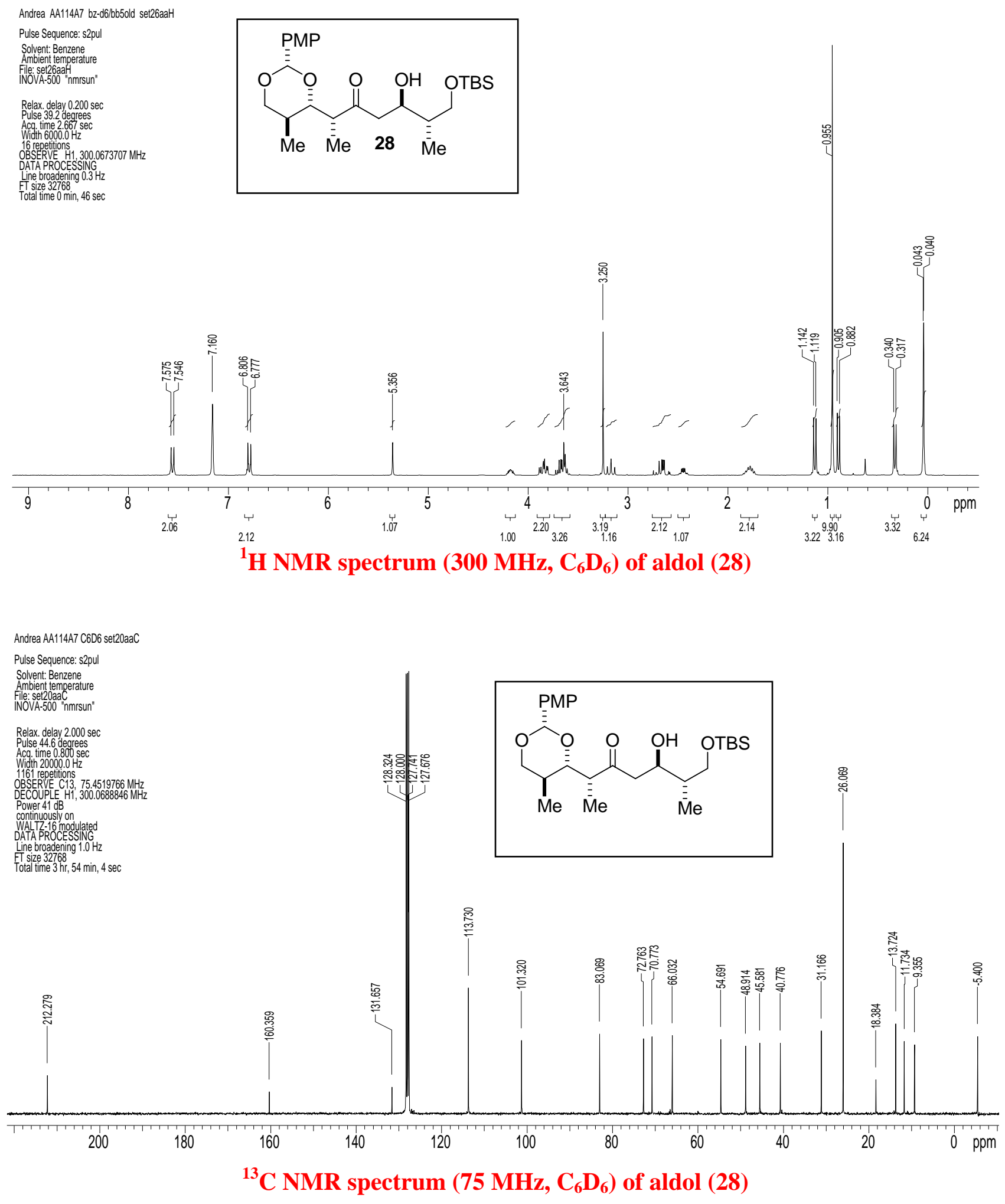


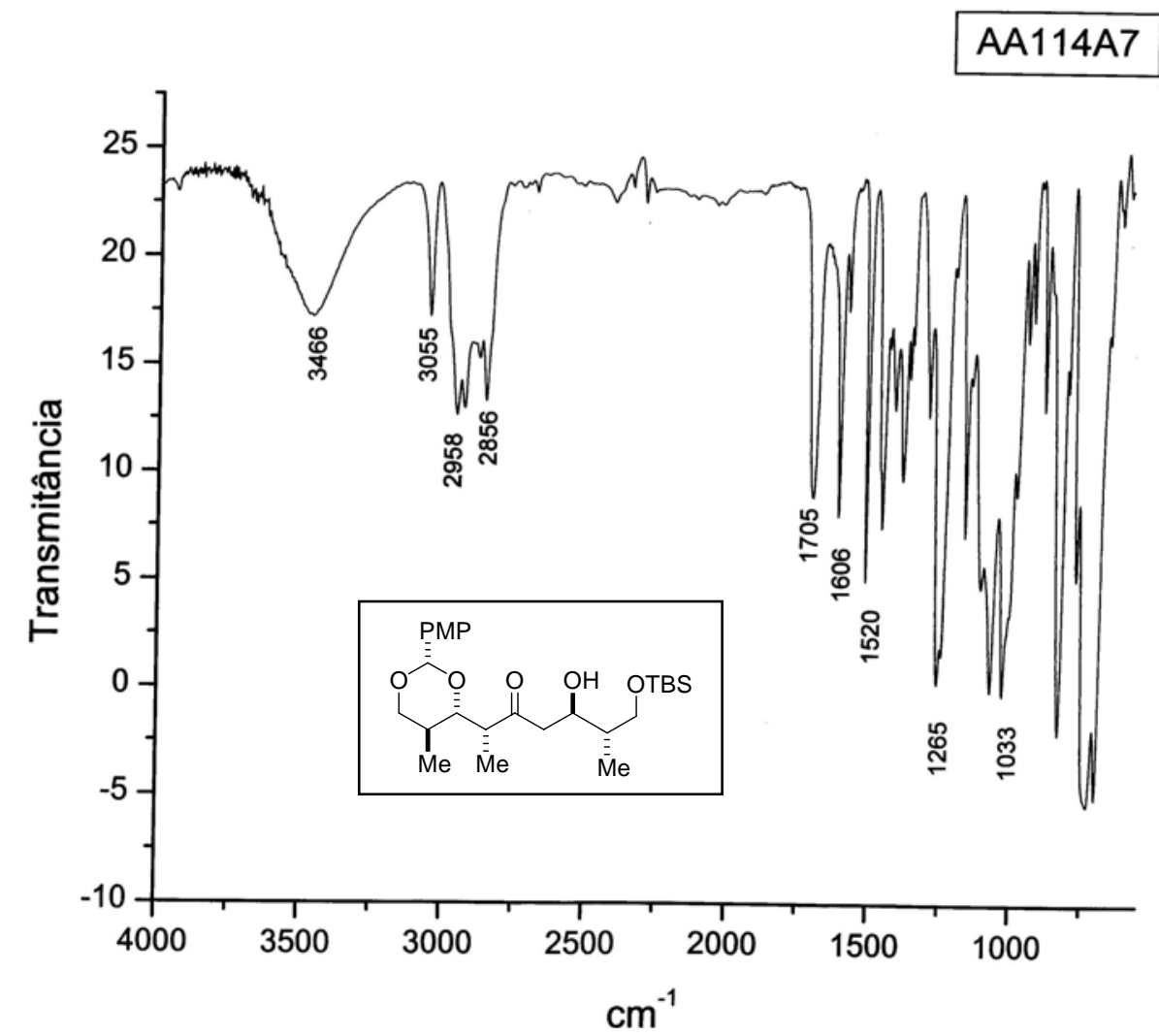

IR spectrum of aldol (28)

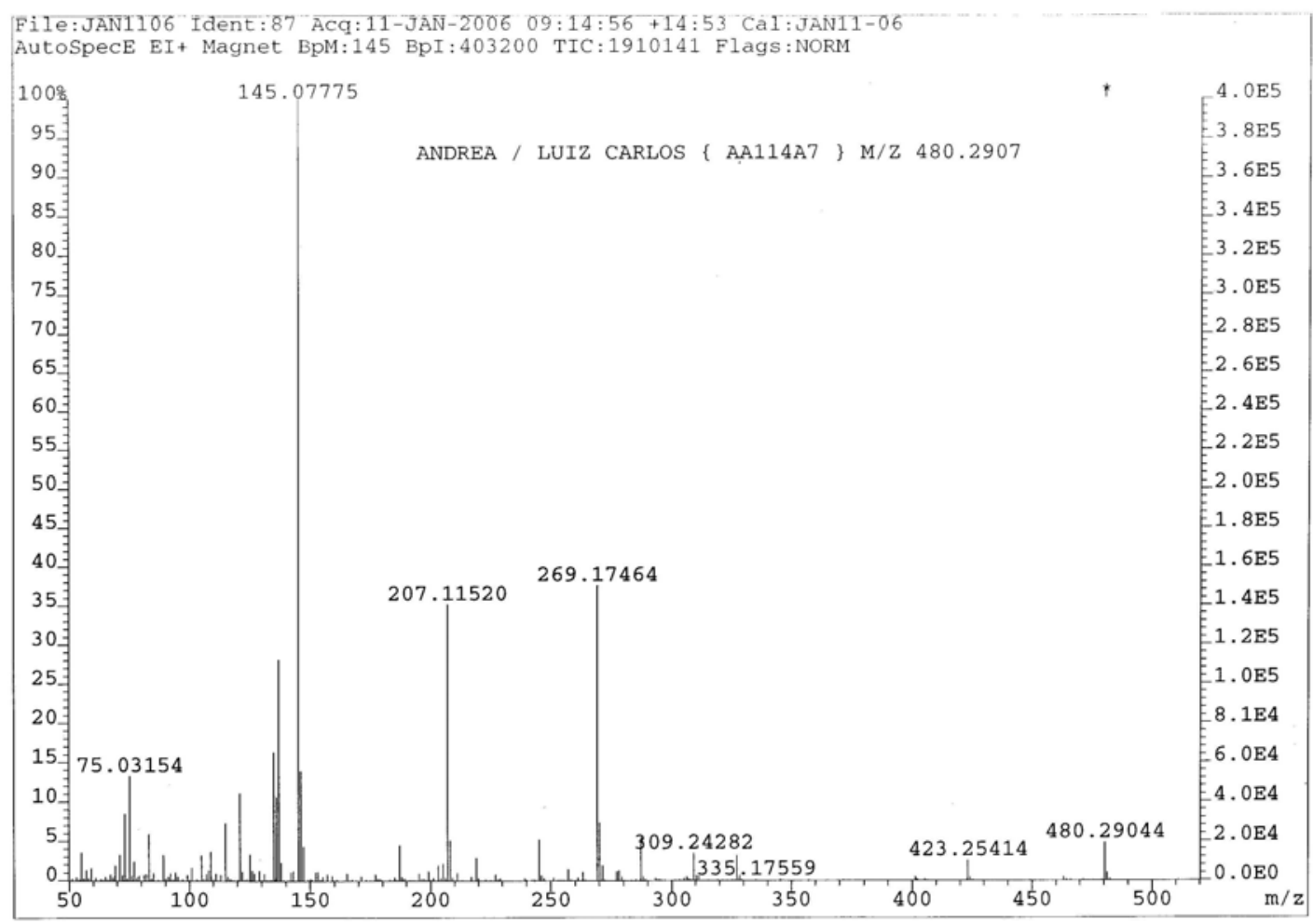

HRMS of aldol (28) 


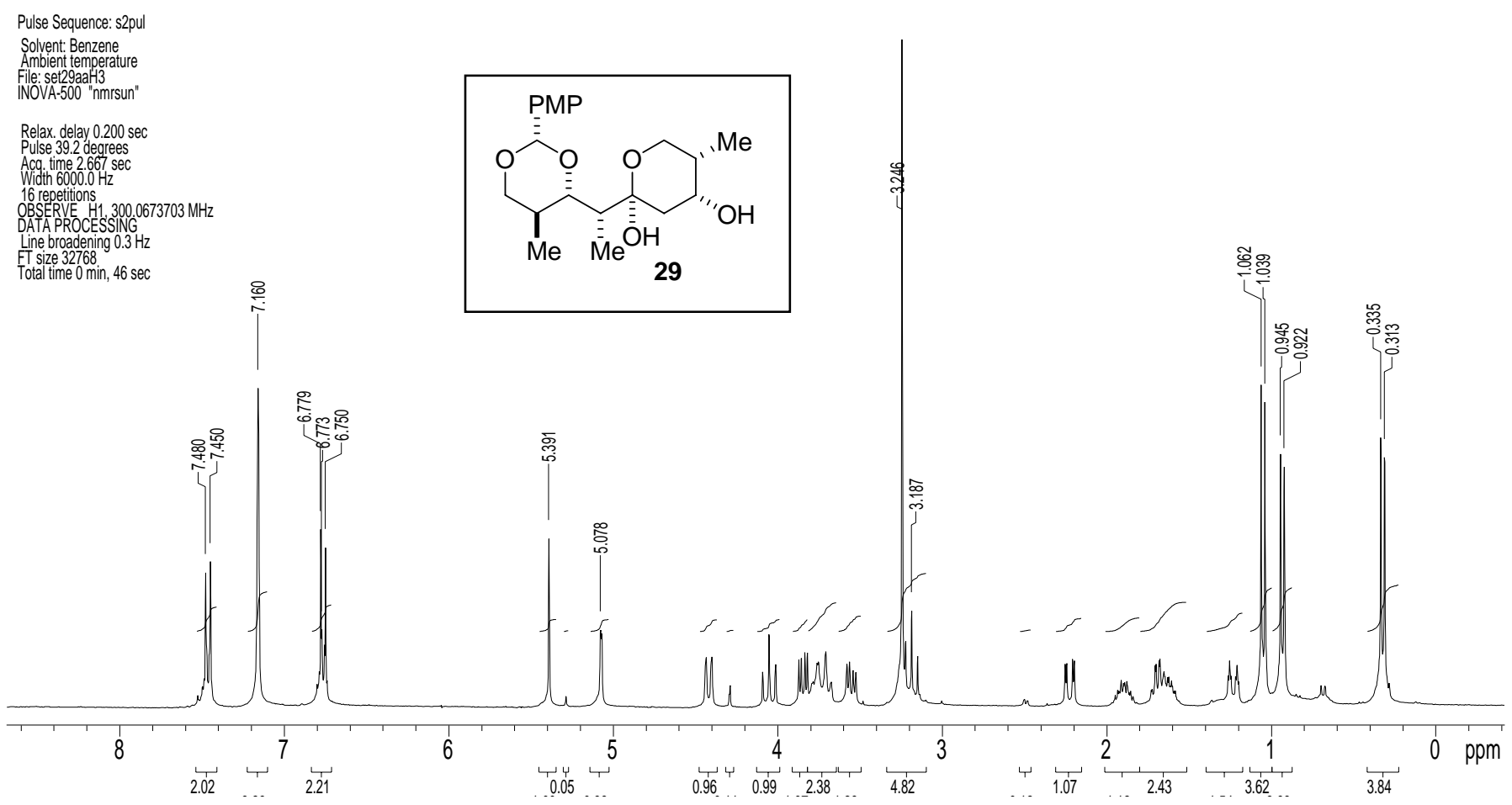

${ }^{1} \mathrm{H}$ NMR spectrum (300 MHz, $\mathrm{C}_{6} \mathrm{D}_{6}$ ) of compound (29)

Andrea AA120A9 C6D6 set29aaC

Pulse Sequence: s2pul

Solvent: Benzene

Ambient temperature
File: set29aaC
NNOVA-500 "nmrsun"

nova-500 "nmrsun"

Relax. delay $2.000 \mathrm{sec}$
Pulse 44.6 degrees
Acg time 0.800 sec

Acc. time $0.800 \mathrm{sec}$

8476 repettitions $75.9519766 \mathrm{MHz}$

DECOUPLE H1', 300.0688846 MHz

Power $41 \mathrm{~dB}$
continuously on

Continuously on
WATLZ16 modulated
DATA PROCESSING

Line broadering $1.0 \mathrm{~Hz}$

Total time $15 \mathrm{hr}, 36 \mathrm{~min}, 16 \mathrm{sec}$
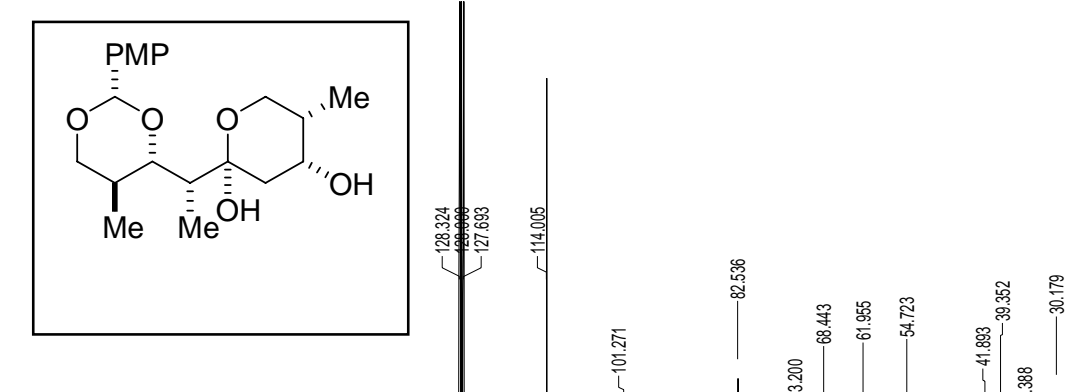

䦖

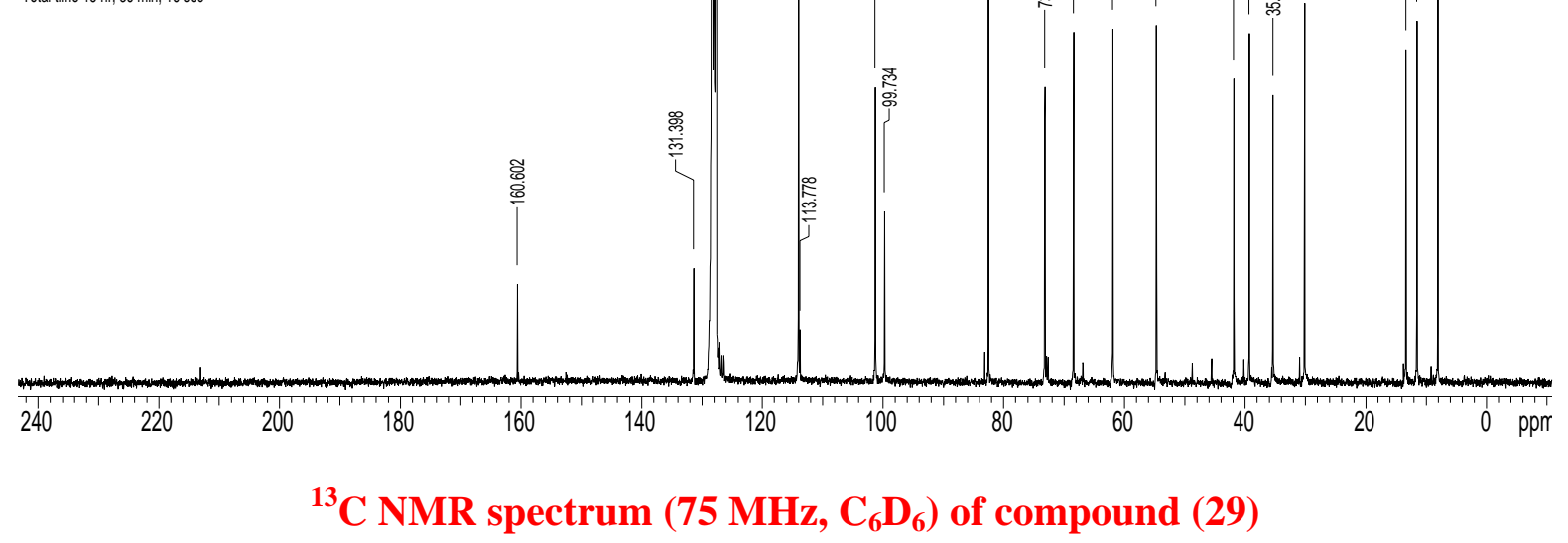




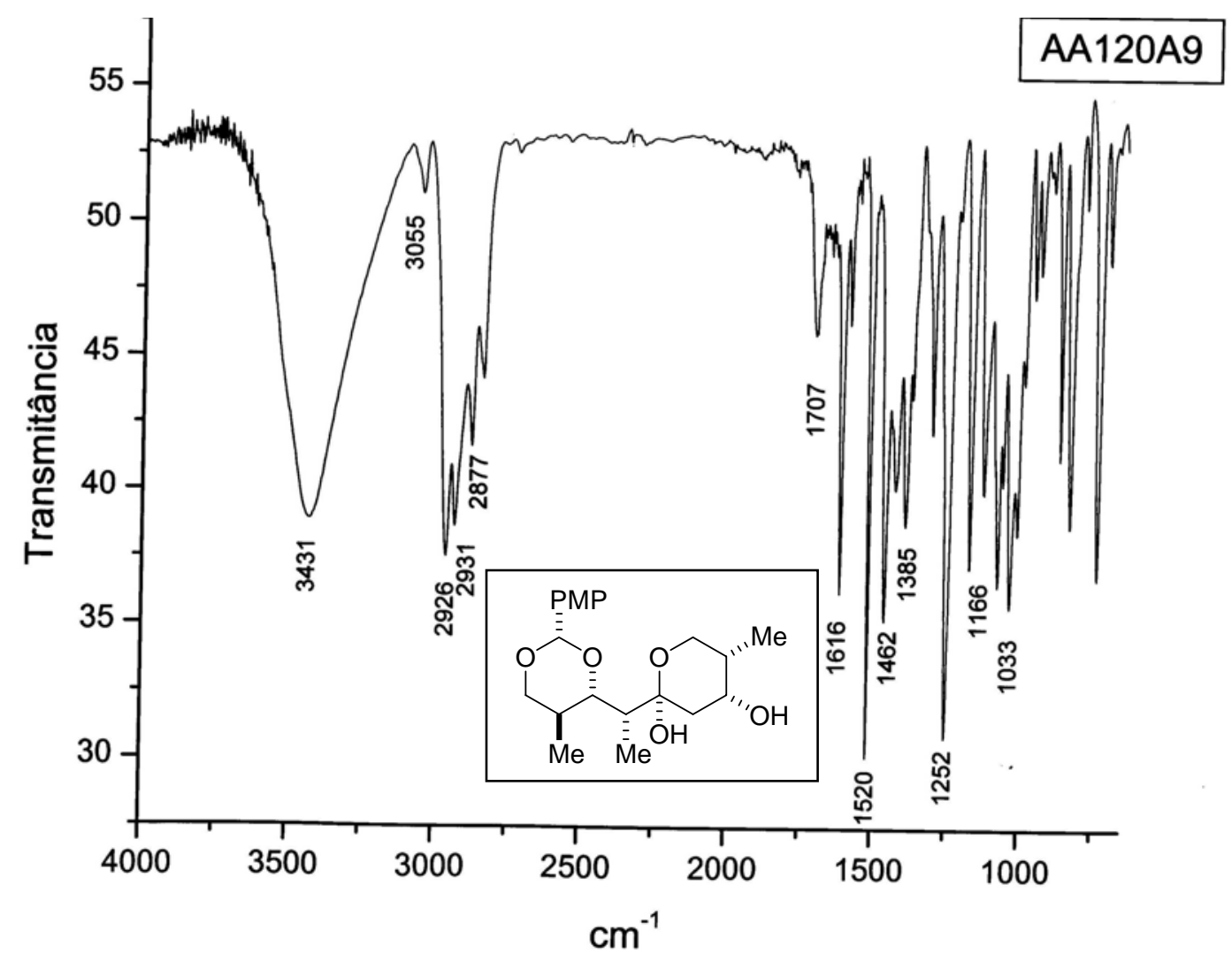

IR spectrum (film) of compound (29)

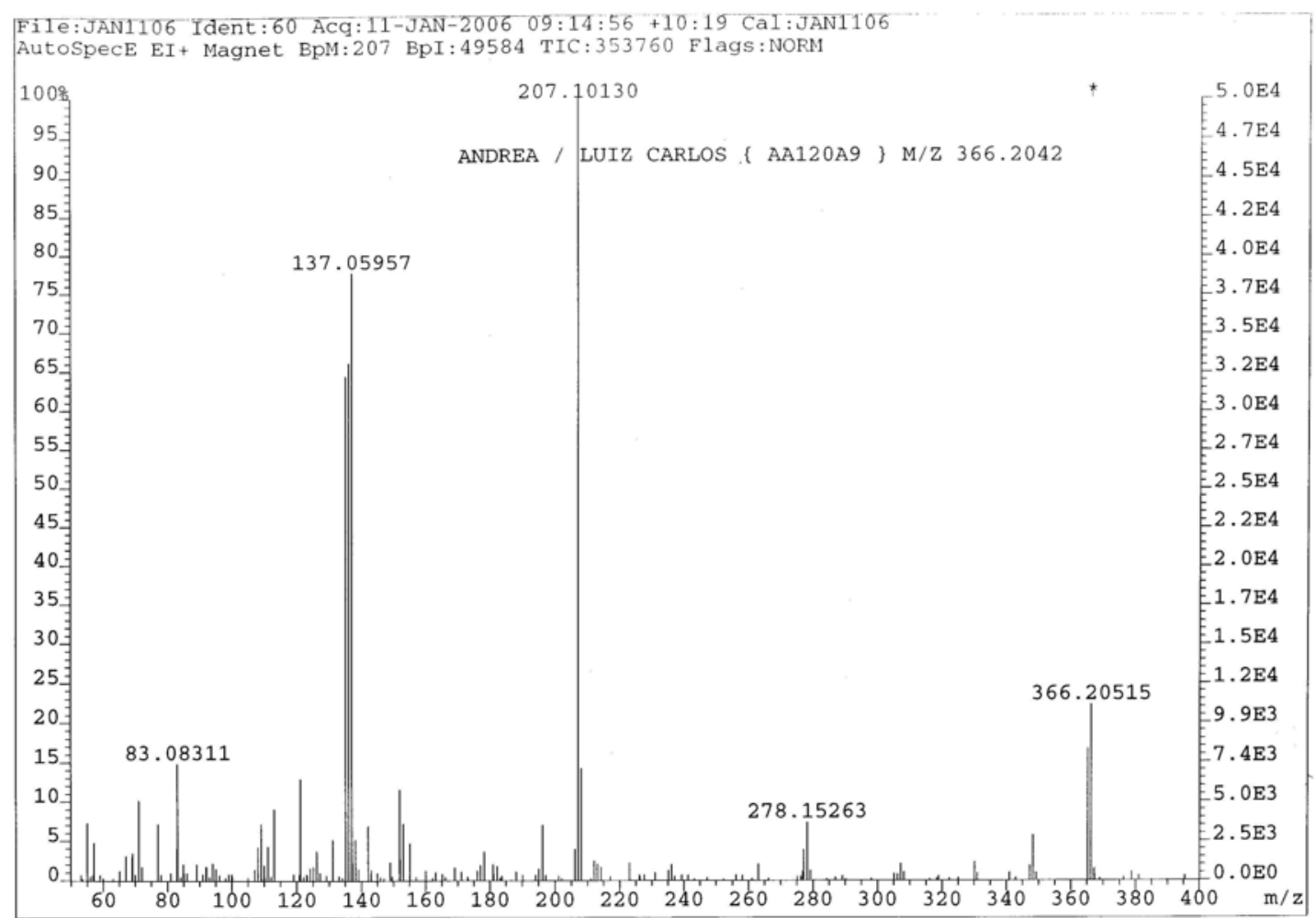

HRMS of compound (29) 
(2R,5R,6R)-7-(tert-butyldimethylsilyloxy)-5-hydroxy-2-((2S,4S,5S)-2-(4-methoxyphenyl)-5methyl-1,3-dioxan-4-yl)-6-methylheptan-3-one (30):
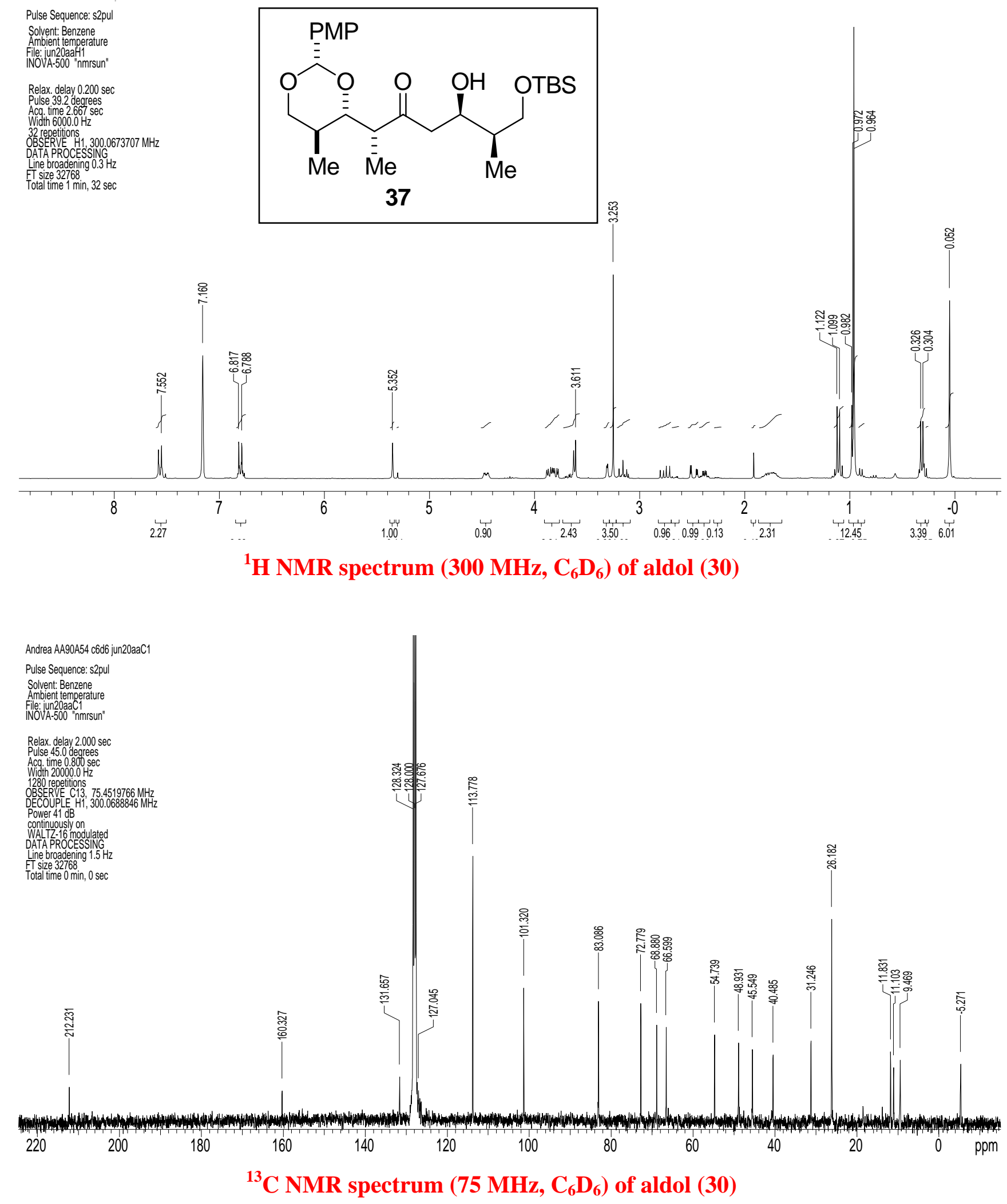

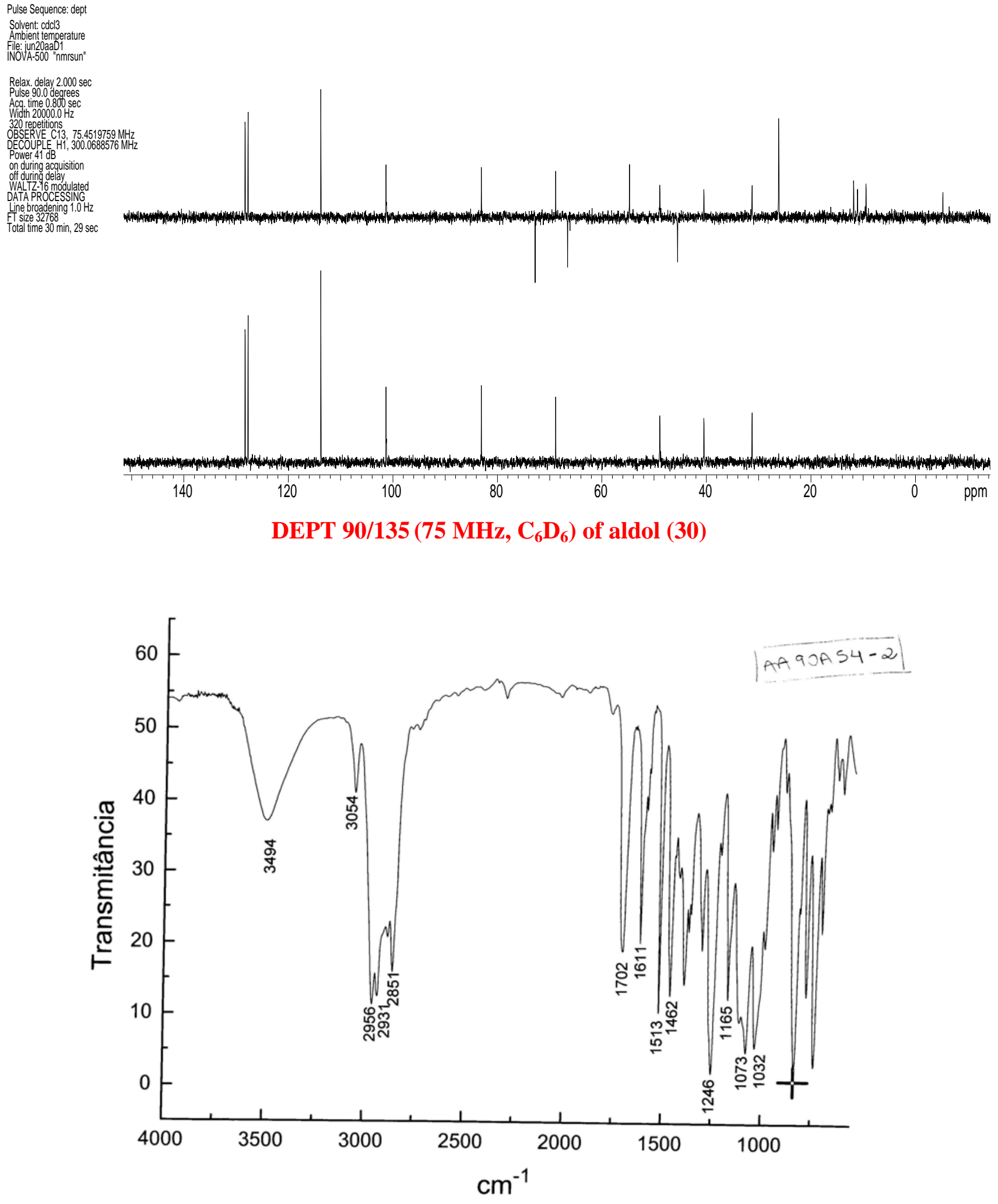

IR spectrum of aldol (30) 


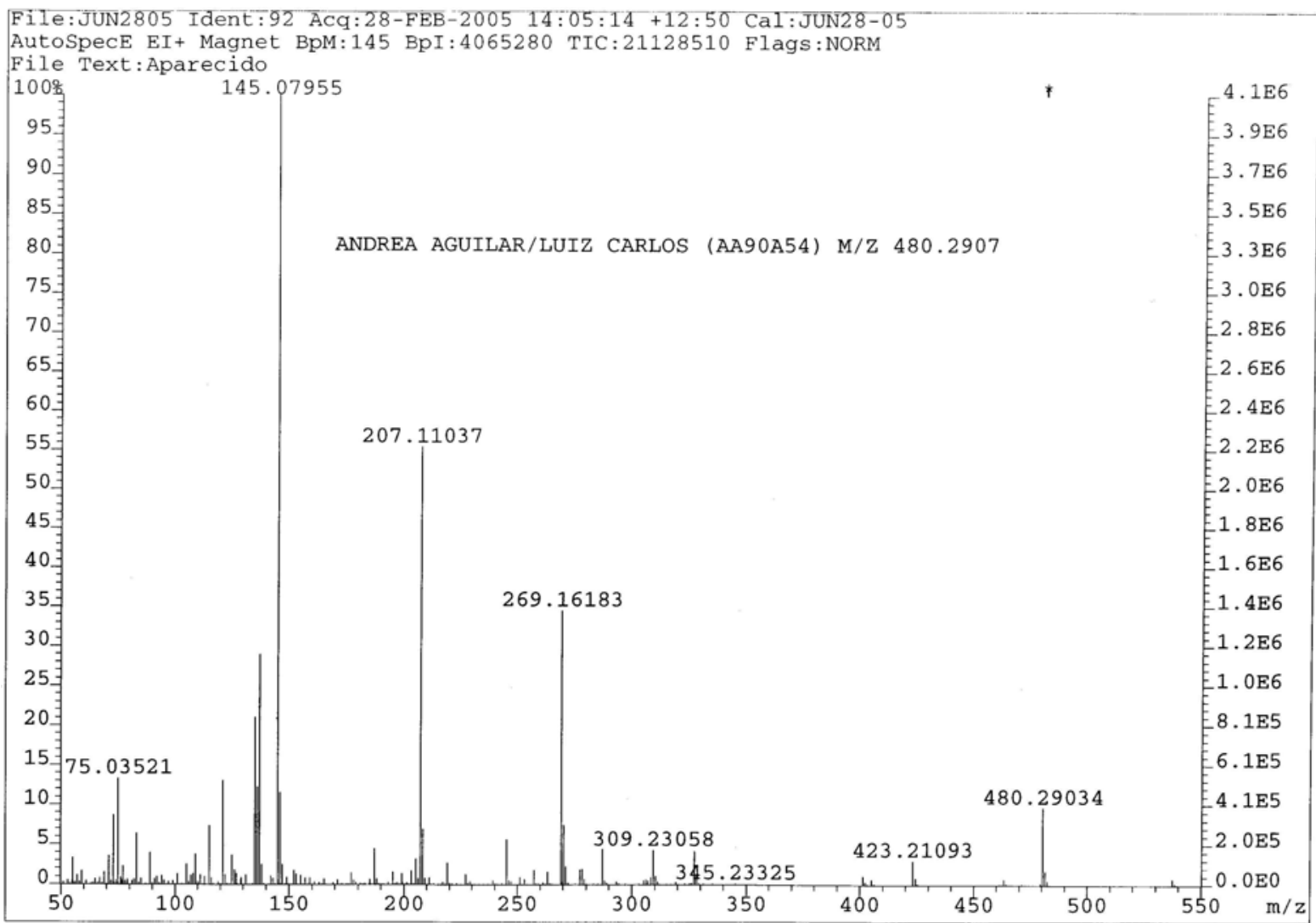

HRMS of aldol (30)

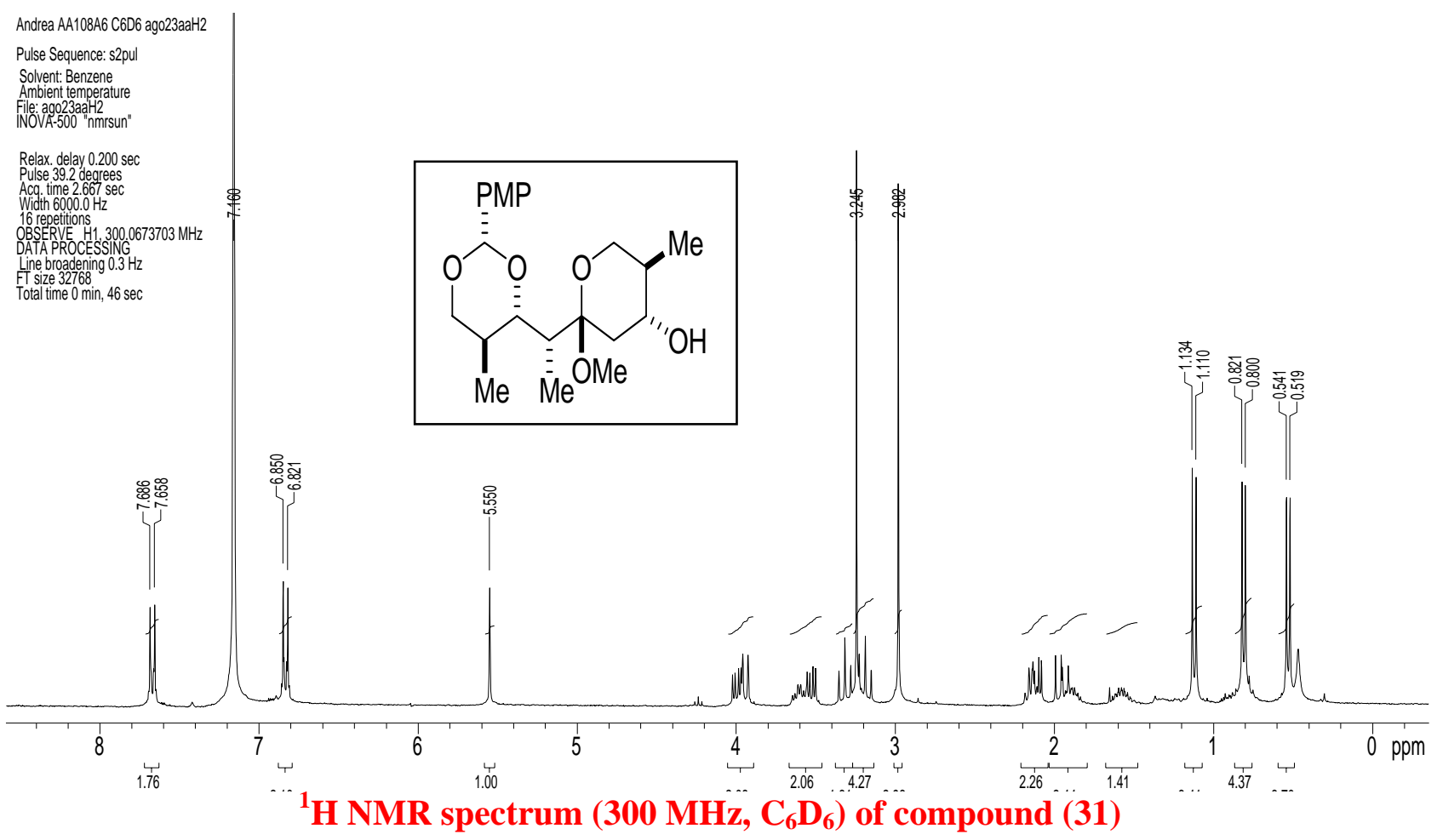



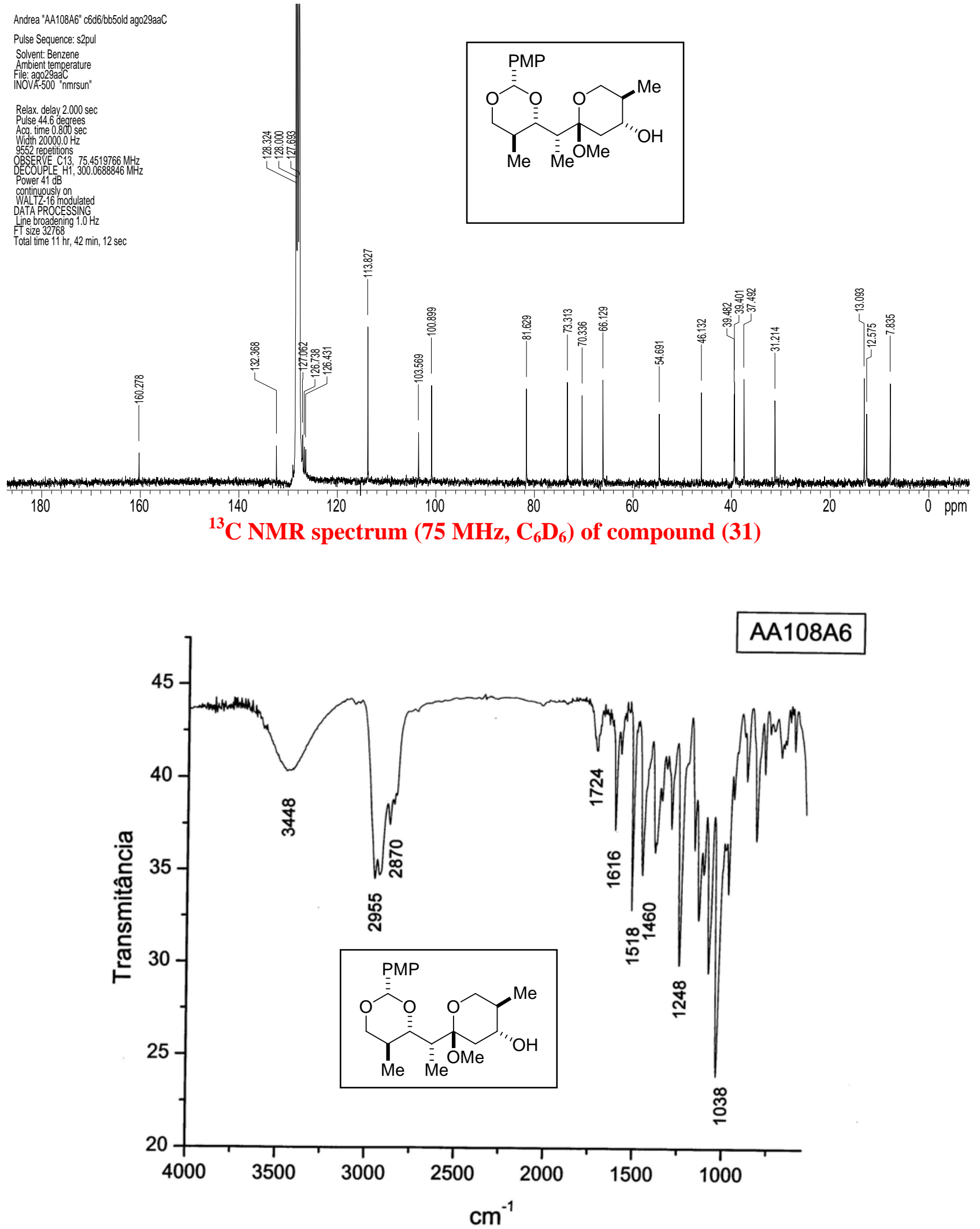

IR spectrum of compound (31) 


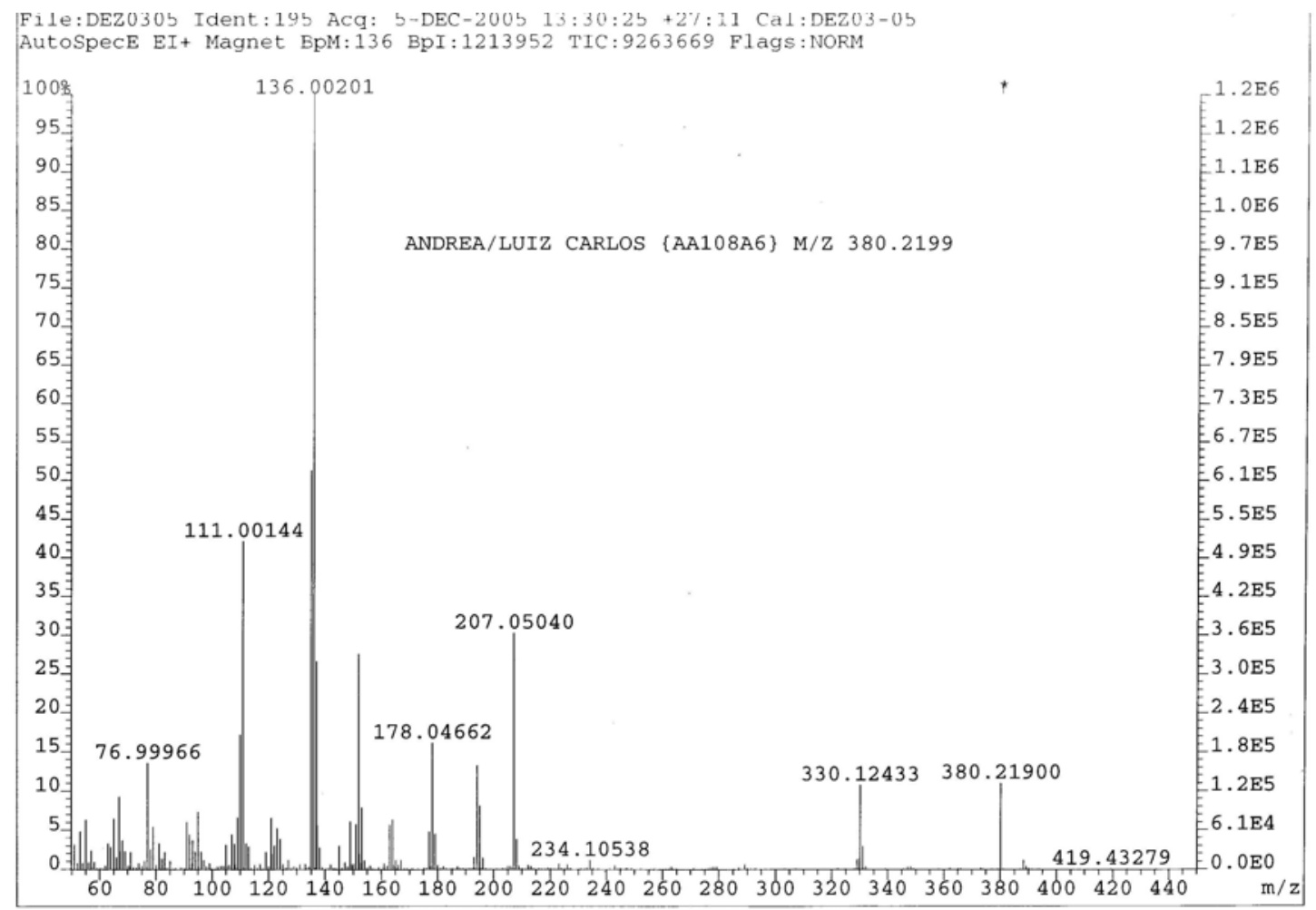

HRMS of compound (31) 


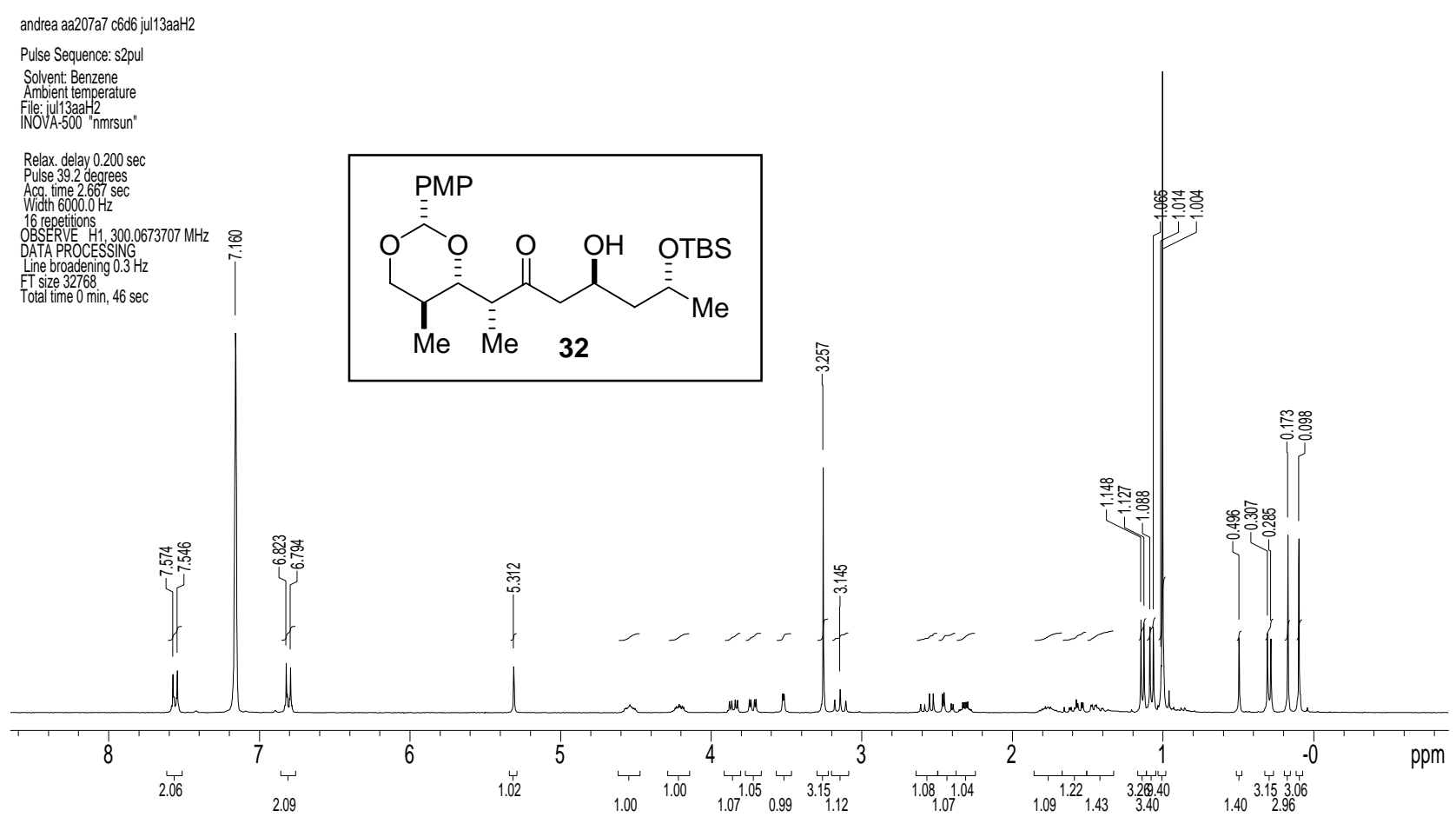

${ }^{1} \mathrm{H}$ NMR spectrum (300 MHz, $\mathrm{C}_{6} \mathrm{D}_{6}$ ) of aldol (32, major isomer)
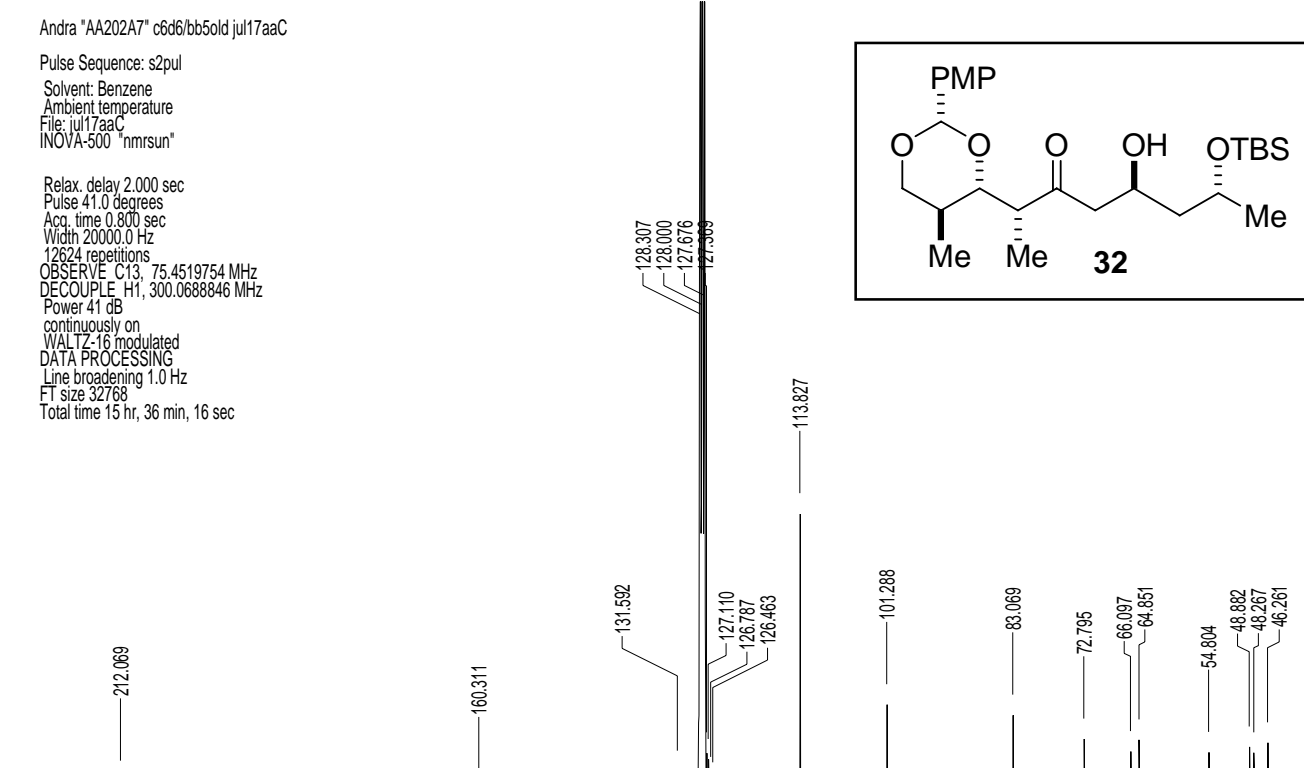

${ }^{13} \mathrm{C}$ NMR spectrum (75 MHz, $\mathrm{C}_{6} \mathrm{D}_{6}$ ) of aldol (32, major isomer) 
SI-30

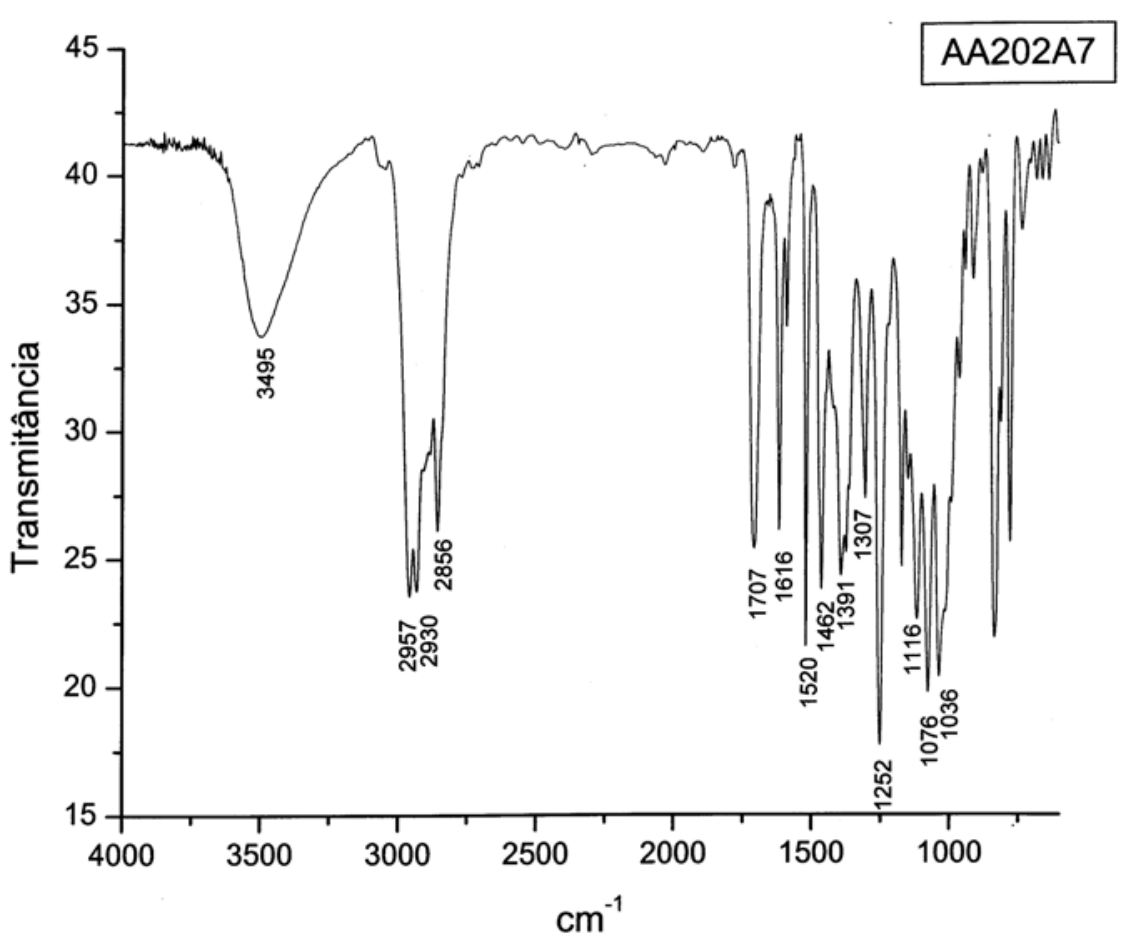

andrea aa202a23-2 c6d6 jun29aaH1

IR spectrum (film) of aldol (32, major isomer)

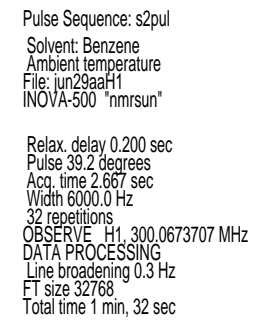

Solvent: Benzene

Ambient temperature

inOVA-500 "nmrsun"

Relax. delay $0.200 \mathrm{sec}$
Pulse 392 degres

Pulse 39.2 degrees

Width $6000.0 \mathrm{~Hz}$

FT size 32768 . 35

al time $1 \mathrm{~min}, 32 \mathrm{sec}$
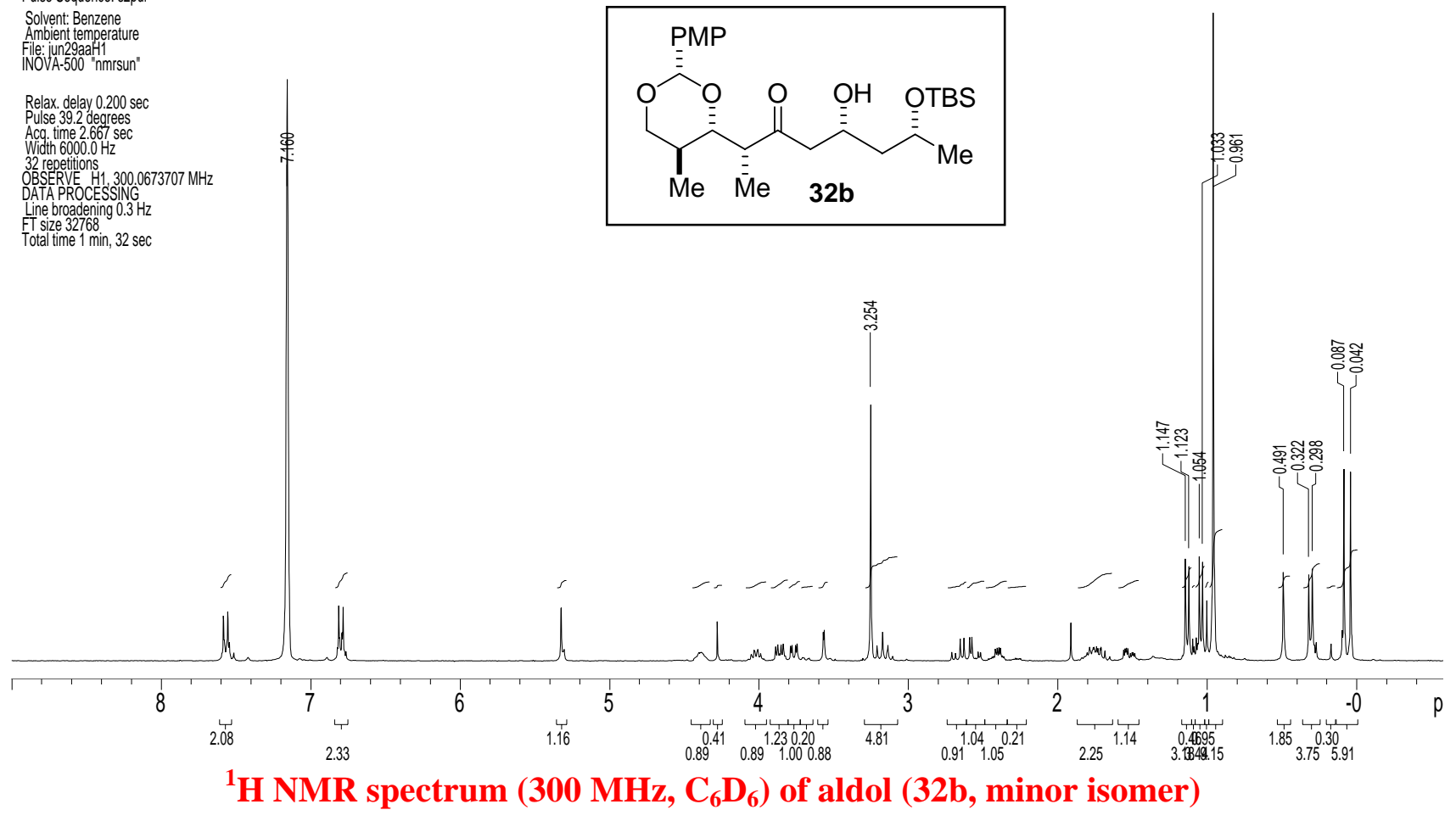
Andrea AA202A23-2 c6d6 jun30aaC

Pulse Sequence: s2pul

Solvent: Benzene

Ambient temperatu

File: jun30aa
INOVA-500 "nmrsun"

Relax. delay $1.500 \mathrm{sec}$

Pulse 45.0 degrees

Acq. ime 0.965 sec

35000 repetitions

OBSERVE C13, $125.6955663 \mathrm{MH}$

DECOUP LE H1, $499.8852083 \mathrm{MH}$

Power $37 \mathrm{~dB}$

continuously on

DATA PROCESSING

Line broadening $1.0 \mathrm{~Hz}$

Total time $24 \mathrm{hr}, 3 \mathrm{~min}, 6 \mathrm{sec}$

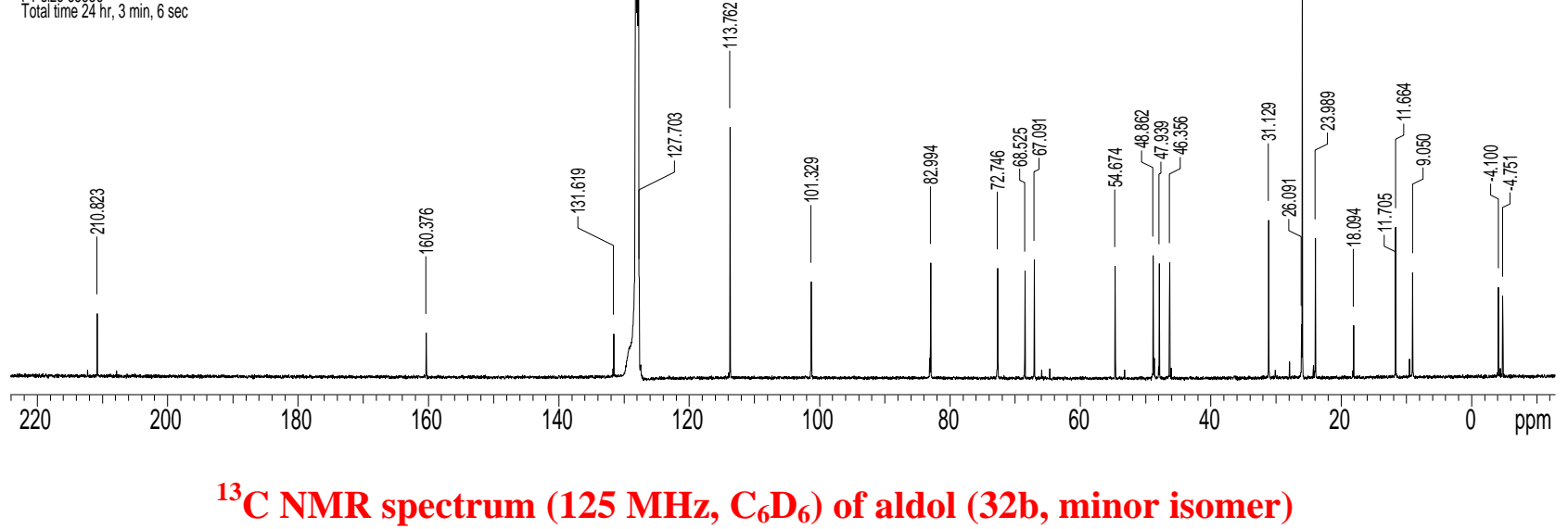

${ }^{13} \mathrm{C}$ NMR spectrum (125 MHz, $\mathrm{C}_{6} \mathrm{D}_{6}$ ) of aldol (32b, minor isomer)

\section{AA202A23}

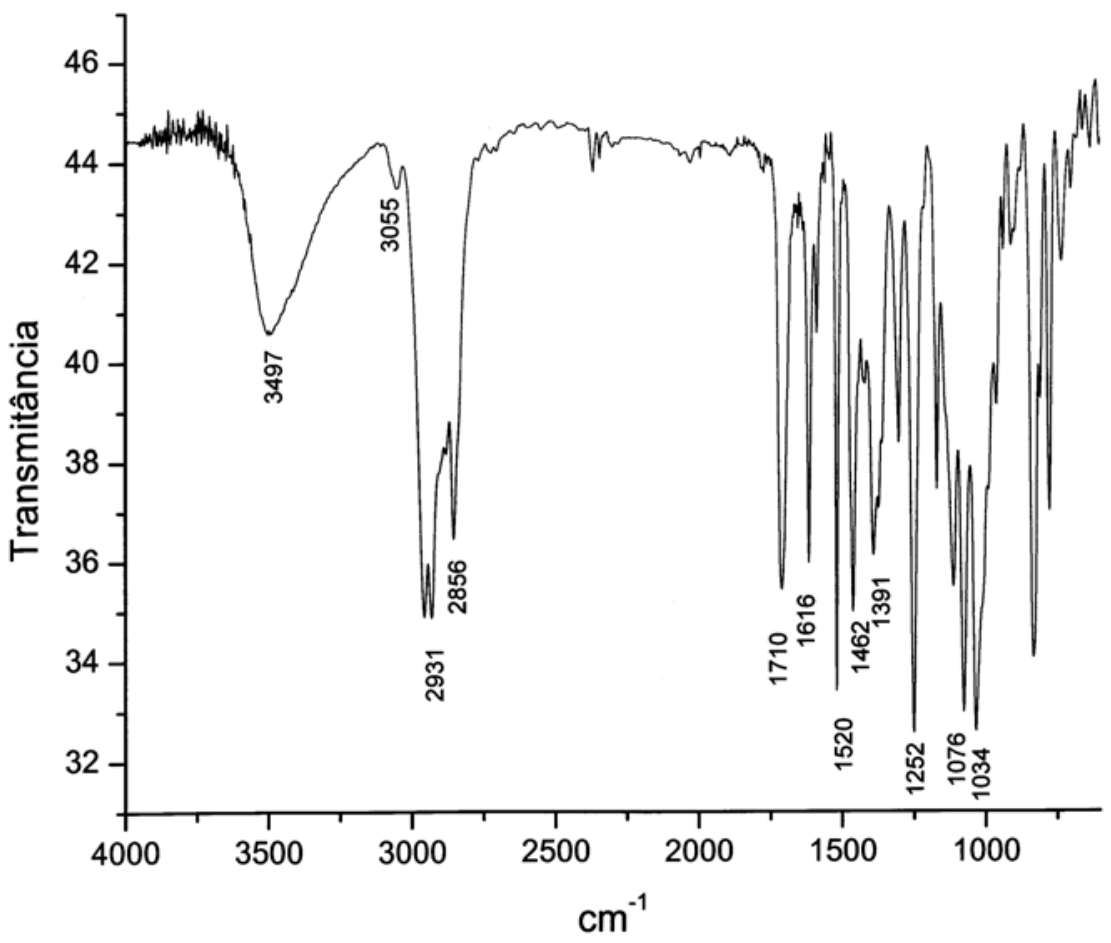

IR spectrum (film) of aldol (32b, minor isomer) 


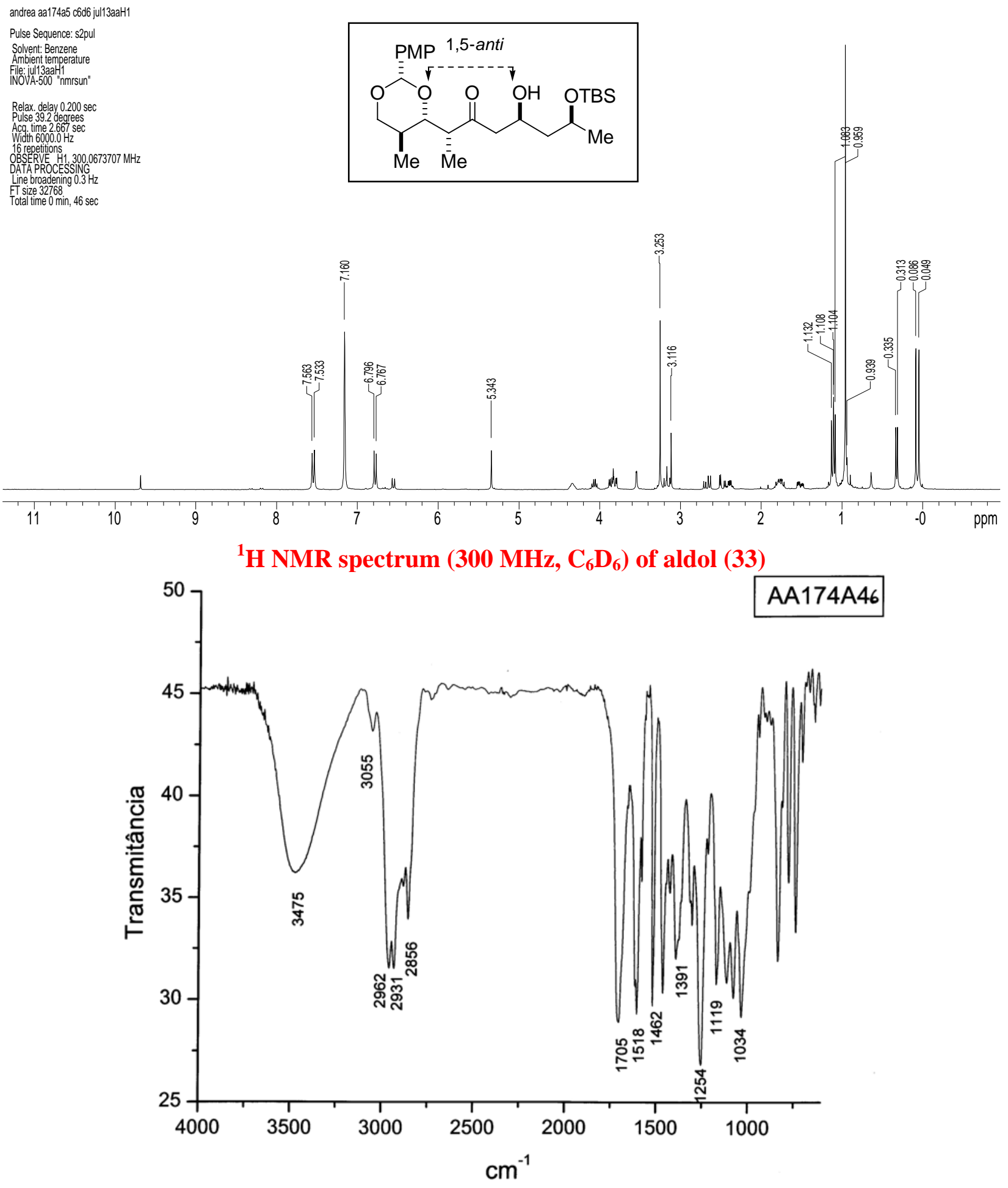

IR spectrum of aldol (33) 
SI-33
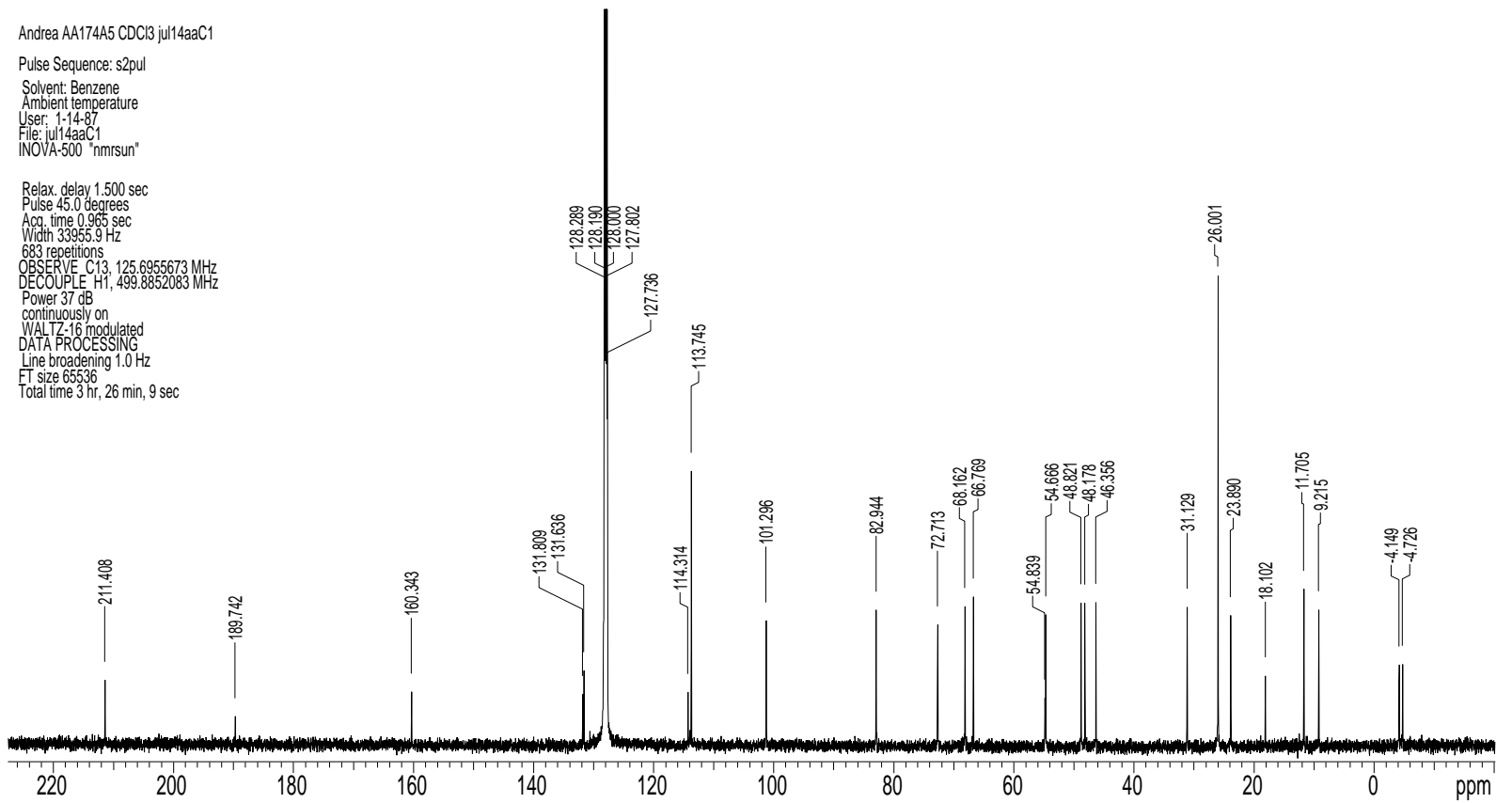

${ }^{13} \mathrm{C}$ NMR spectrum (75 MHz, $\mathrm{C}_{6} \mathrm{D}_{6}$ ) of aldol (33)

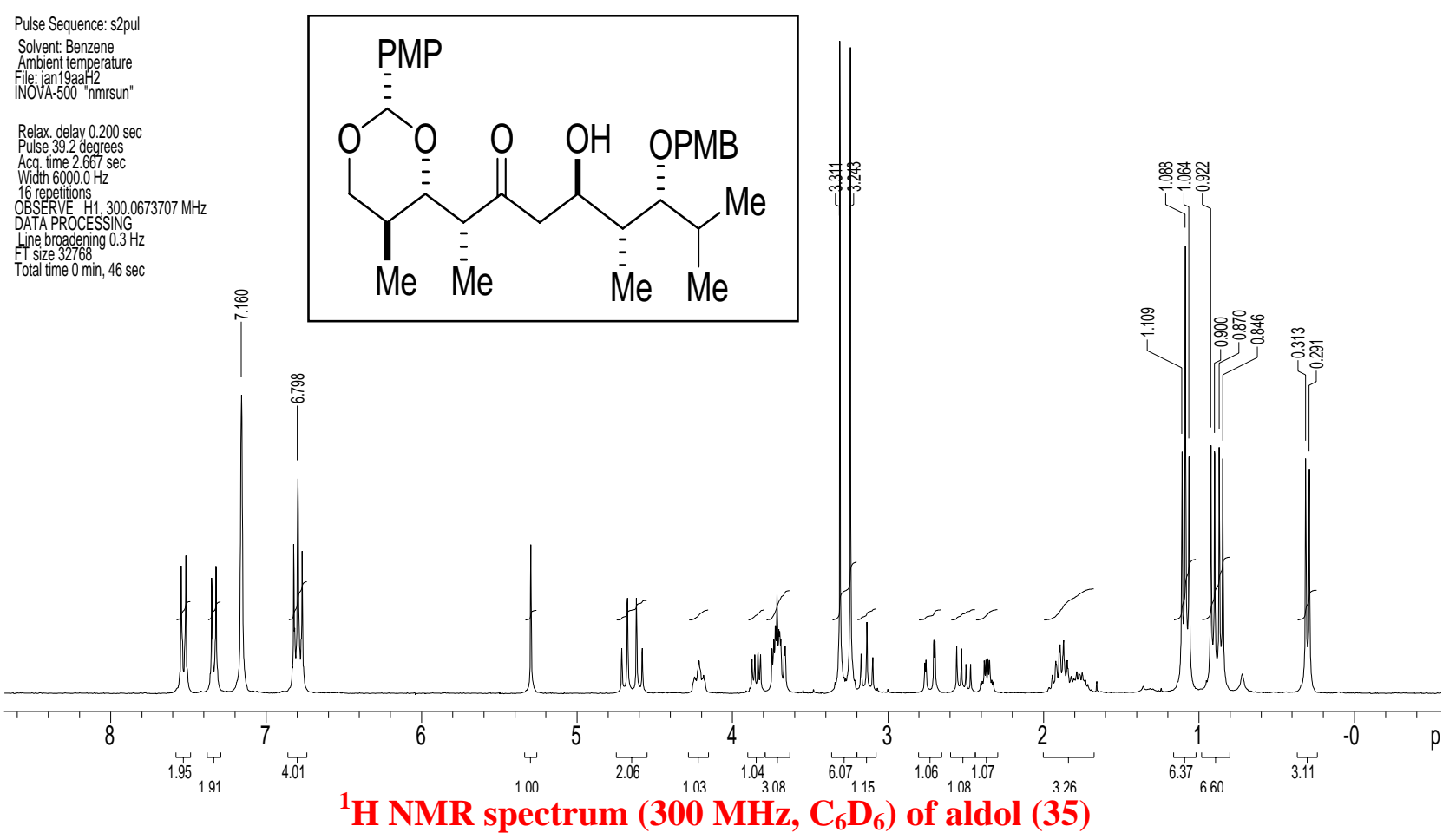


Andrea AA157A39 cdc13 jan23aac

Pulse Sequence: s2pul

Solvent: Benzene

Ambient temperature
User: 1-14-87

File: jan23aaC
INOVA-500 "nmrsun"

Relax. delay $1.500 \mathrm{sec}$
Pulse 45.0 dearees

Acq. time $1.040 \mathrm{sec}$
Width $33955.9 \mathrm{~Hz}$

928 repetitions $125.6955670 \mathrm{MHz}$

BSEREE C13, $125.6955670 \mathrm{MHZ}$
DECOUPLE H1, 499.8852083 MHz
Power 37 dB

continuously on

DATA PROCESSING

Line braadening $1.0 \mathrm{~Hz}$
FT size 131072 .
Total time $3 \mathrm{hr}, 32 \mathrm{~min}, 24 \mathrm{se}$

hr, $32 \mathrm{~min}, 24 \mathrm{sec}$

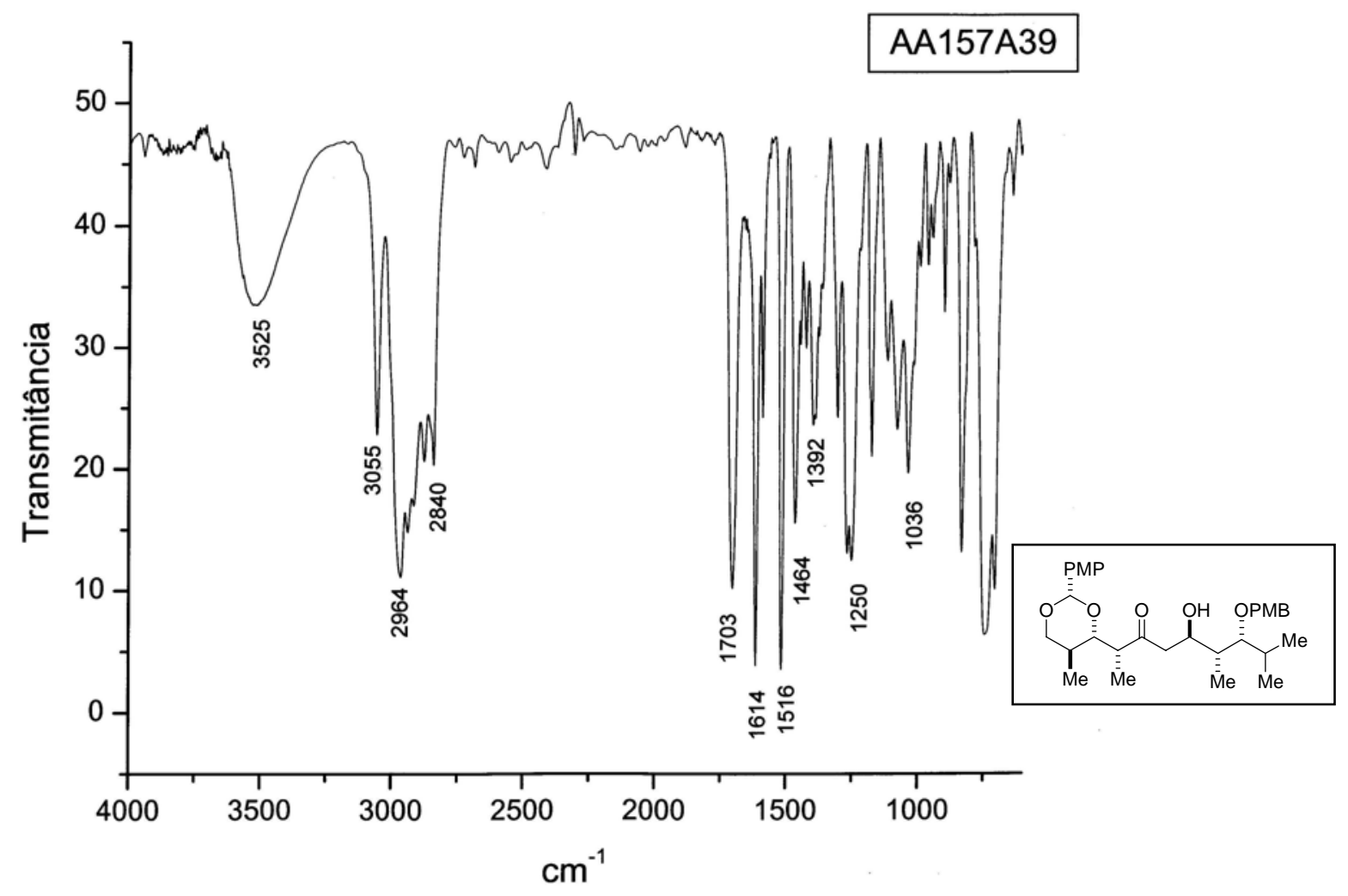

IR spectrum (film) of aldol (35) 


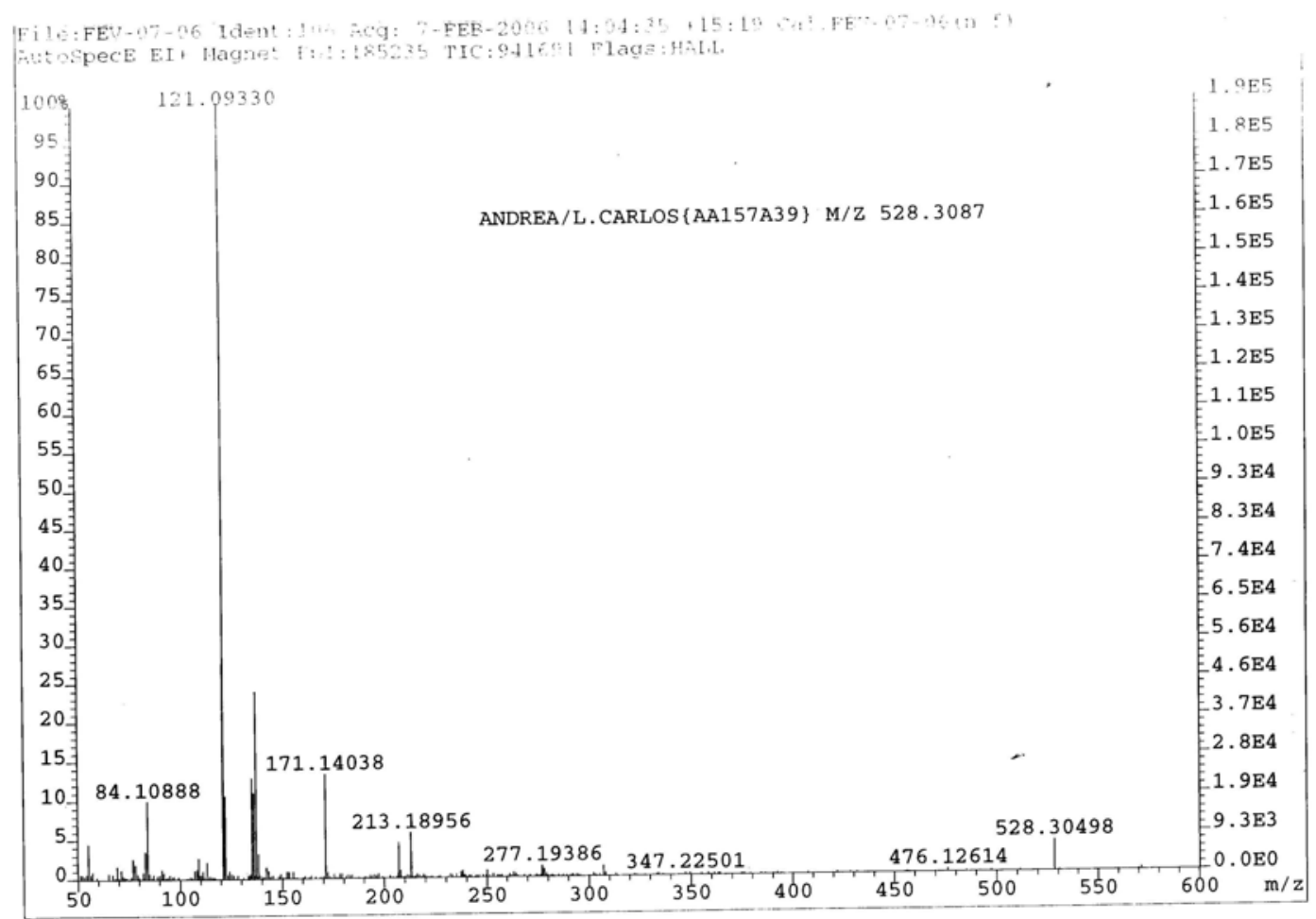

HRMS of aldol (35)

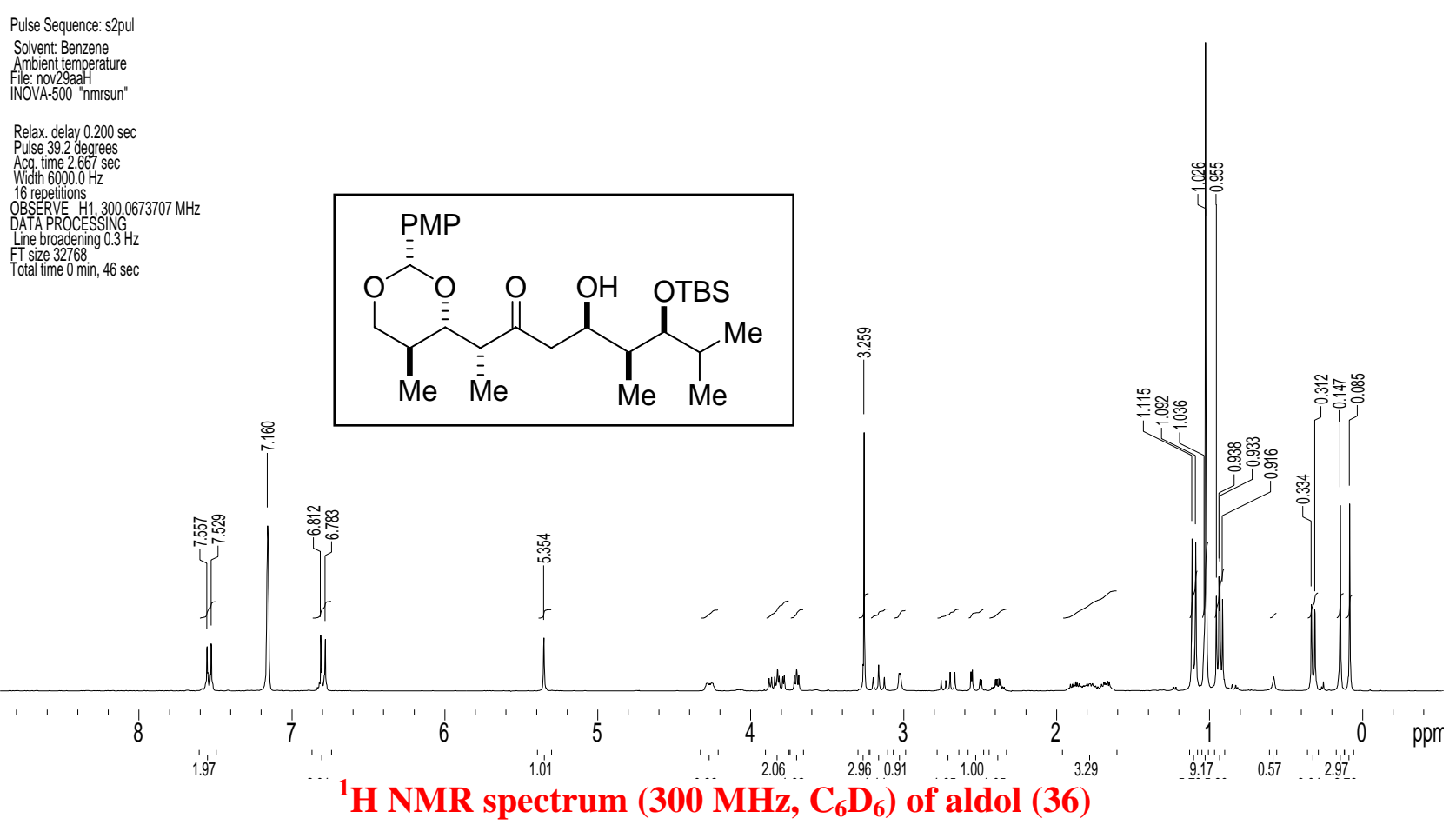


Andrea "AA141A21" c6d6/bb5old nov29aaC

Pulse Sequence: s2pul

Solvent: Benzene

Ambient temperature

File: nov29aac
INOVA-500 "nmrsun"

Relax. delay $2.000 \mathrm{sec}$

Acc. time $0.800 \mathrm{sec}$

Width $20000.0 \mathrm{~Hz}$

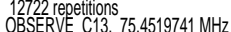

DECOUPLE H1, $300.0688846 \mathrm{MHz}$

Power $41 \mathrm{~dB}$

continuously on

WALTZ-16 modulated

DATA PROCESSING

Line broadening $1.0 \mathrm{~Hz}$
FT size 32768

otal time $15 \mathrm{hr}, 36 \mathrm{~min}, 16 \mathrm{sec}$
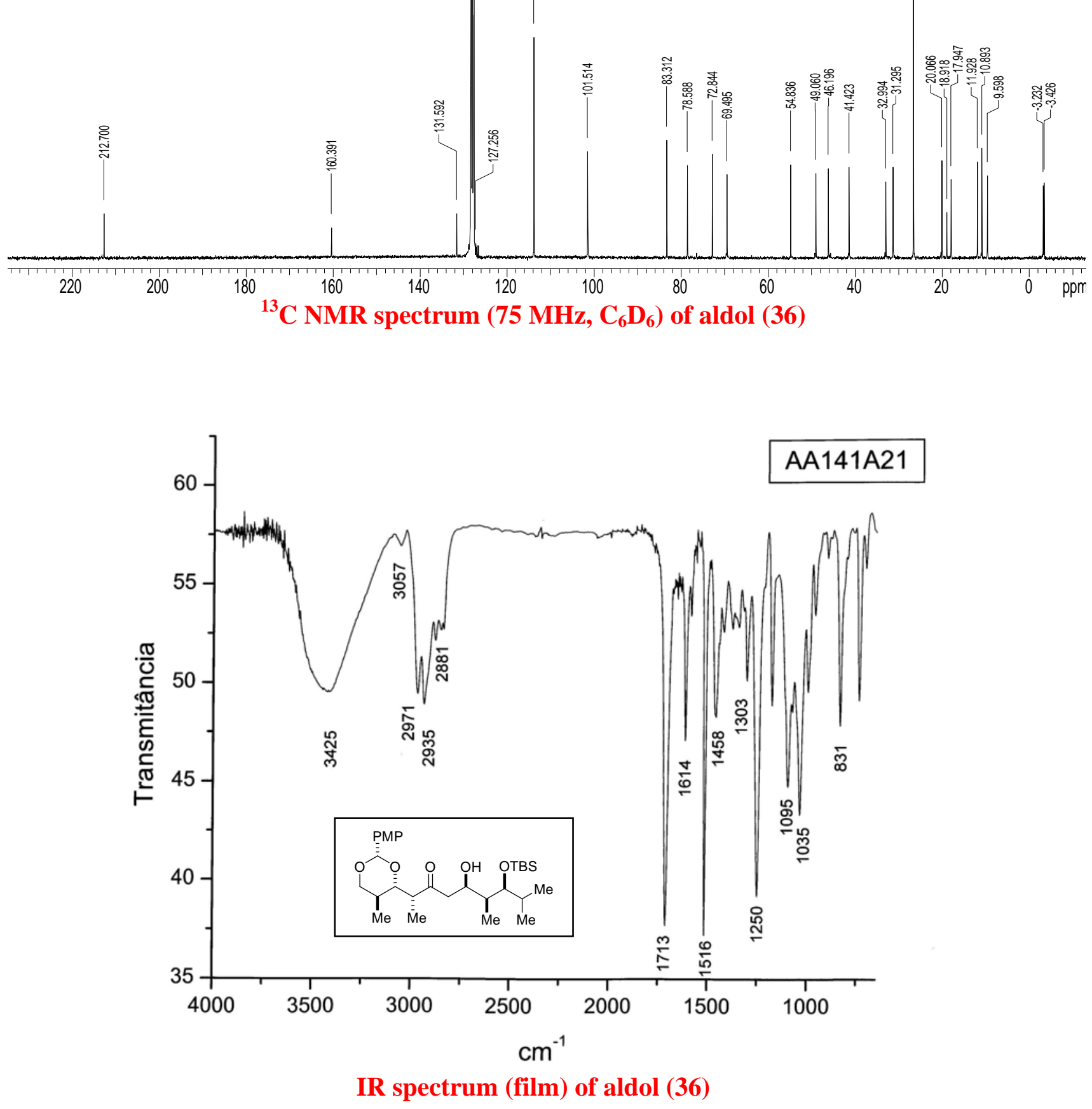


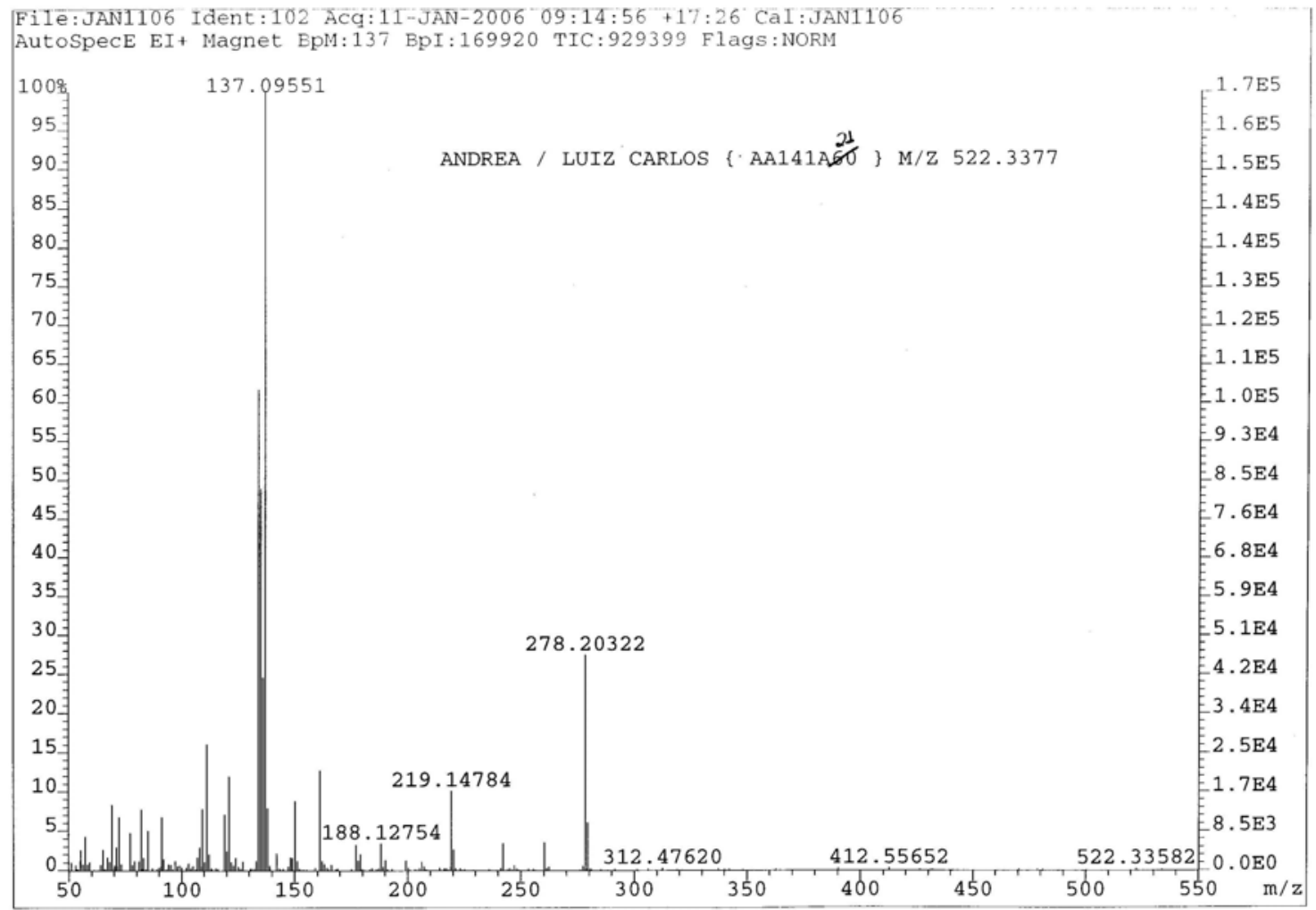

HRMS of aldol (36)

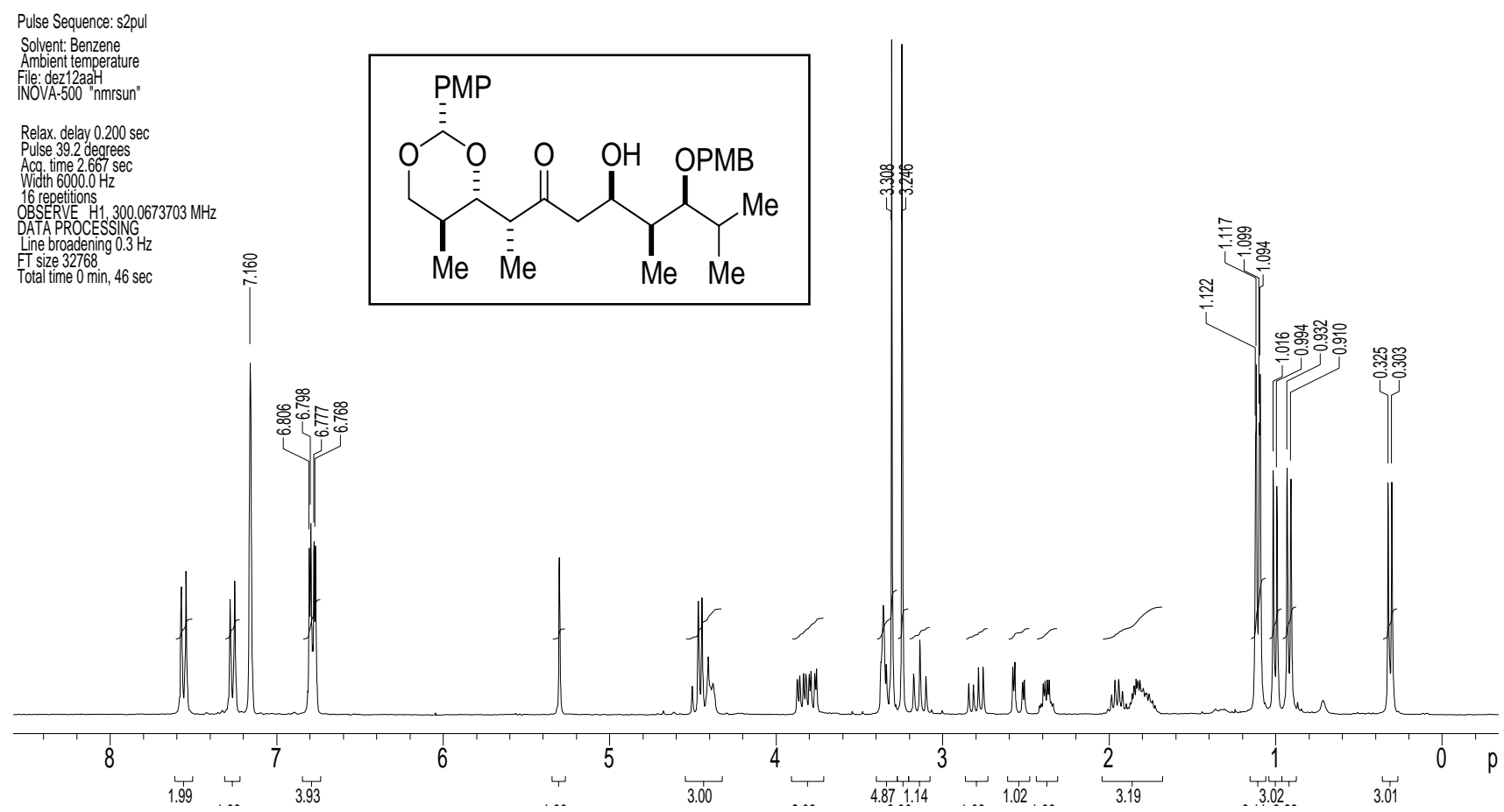

${ }^{1} \mathrm{H}$ NMR spectrum (300 MHz, $\mathrm{C}_{6} \mathrm{D}_{6}$ ) of aldol (37) 

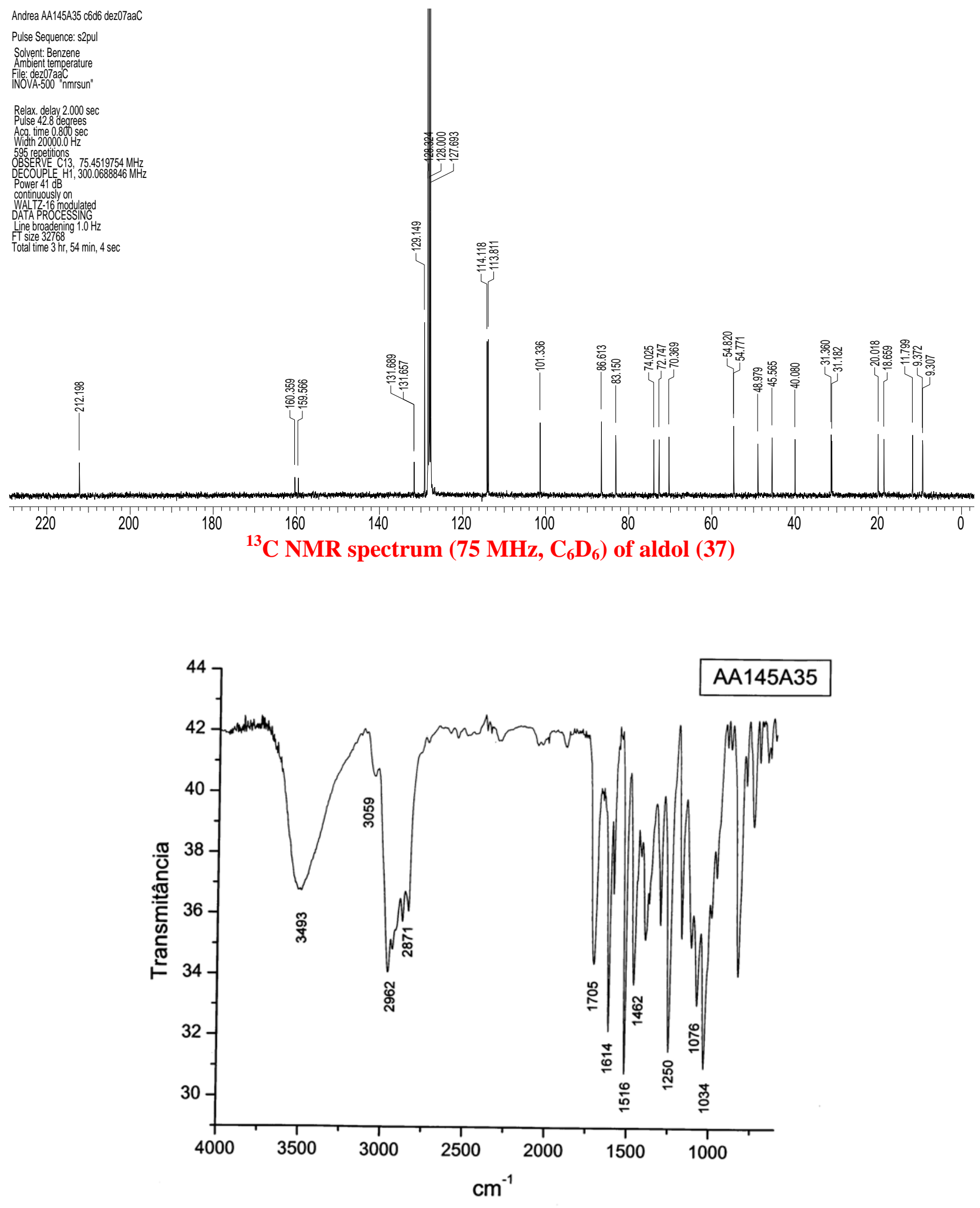

IR spectrum (film) of aldol (37) 


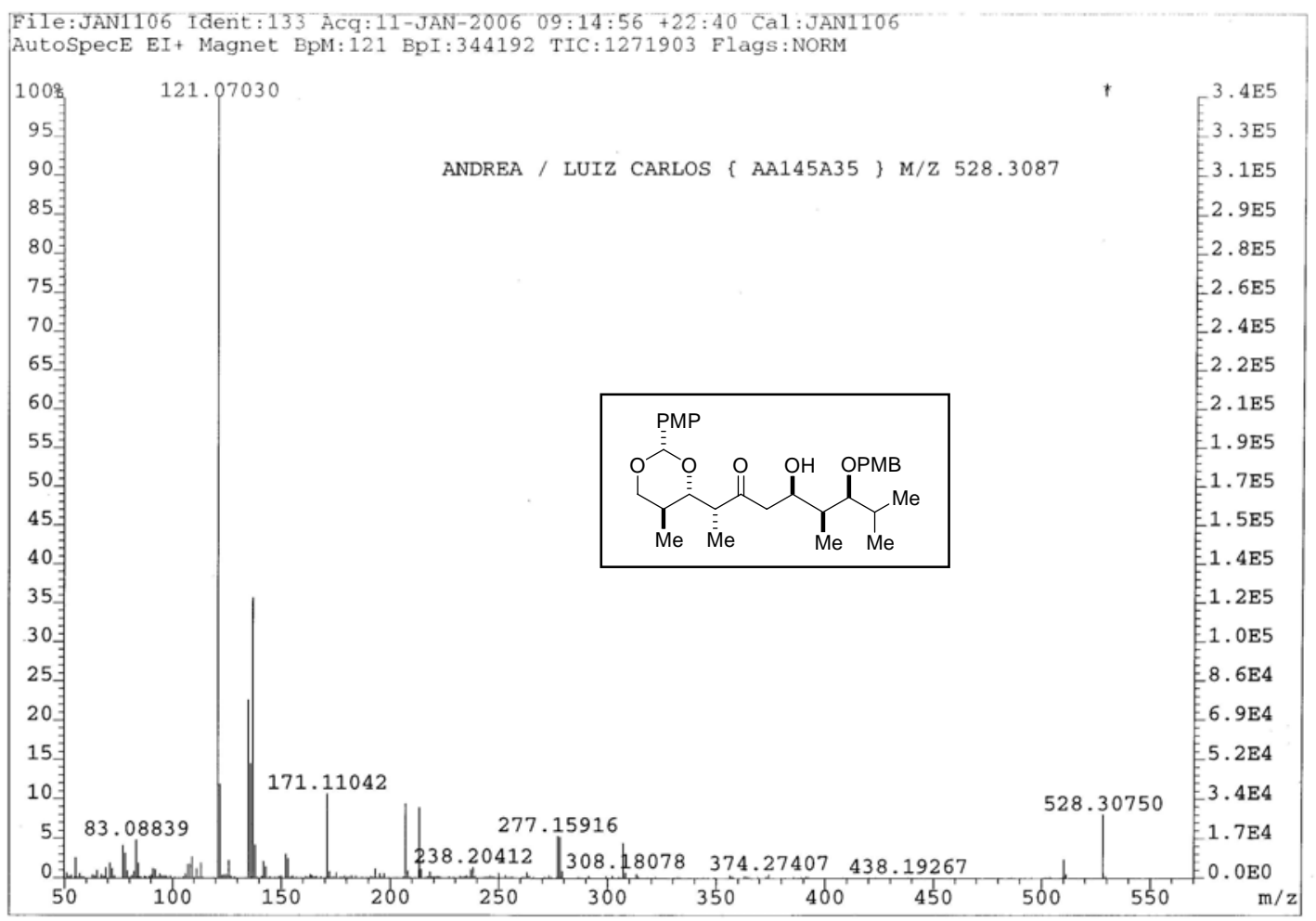

HRMS of aldol (37) 